\title{
The exponentiated Hencky energy: Anisotropic extension and case studies
}

\author{
Jörg Schröder ${ }^{1}$ and Markus von Hoegen ${ }^{1}$ and Patrizio Neff ${ }^{2}$ \\ ${ }^{1}$ Institut für Mechanik, Fakultät für Ingenieurwissenschaften / Abtl. Bauwissenschaften \\ Universität Duisburg-Essen, 45141 Essen, Universitätsstr. 15, Germany \\ e-mail: j.schroeder@uni-due.de, phone: +49 201183 2708, fax: +49 2011832680 \\ e-mail: markus.von-hoegen@uni-due.de, phone: +49 201183 3091, fax: +49 2011832680 \\ ${ }^{2}$ Lehrstuhl für Nichtlineare Analysis und Modellierung, Fakultät für Mathematik \\ Universität Duisburg-Essen, 45127 Essen, Thea-Leymann-Straße 9, Germany \\ e-mail: patrizio.neff@uni-due.de, phone: +49 201183 4243, fax: +49 2011834394
}

\begin{abstract}
In this paper we propose an anisotropic extension of the isotropic exponentiated Hencky energy, based on logarithmic strain invariants. Unlike other elastic formulations, the isotropic exponentiated Hencky elastic energy has been derived solely on differential geometric grounds, involving the geodesic distance of the deformation gradient $\boldsymbol{F}$ to the group of rotations. We formally extend this approach towards anisotropy by defining additional anisotropic logarithmic strain invariants with the help of suitable structural tensors and consider our findings for selected case studies.
\end{abstract}

Keywords: Hencky energy, logarithmic strain tensor, anisotropy, strain invariants, biomechanics

\section{Introduction}

In this article we consider a novel Hencky-type hyperelasticity model, the exponential Hencky-logarithmic strain energy proposed by Neff et al. [46], Neff et al. [47] and Neff and Ghiba [42. Here, we focus on an extension to anisotropy in a coordinate invariant setting. Therefore, we apply the concept of structural tensors and introduce additional mixed invariants. The flexibility of the proposed formulation is demonstrated by identifying the linearized fourth-order elasticity tensor with the well-known coordinate dependent representations. Furthermore, we propose an anisotropic exponential Hencky model suitable for the description of soft biological tissues. The performance of this model is demonstrated by the analysis of a patient specific artery.

The modeling of anisotropic soft tissues in the framework of nonlinear elasticity has made considerable progress in the last decades. From the mathematical side, the polyconvexity condition introduced by John Ball in his seminal paper Ball [4] is a strong mathematical requirement which implies Legendre-Hadamard ellipticity (rank-one convexity) at all deformation gradients $\boldsymbol{F}$. In the early time after its introduction, polyconvexity was exclusively used in the isotropic setting and it was unclear how to extend the framework to anisotropy. In Hartmann and Neff [21] a large variety of isotropic strain energy functions have been discussed. Two of the present authors have been able to solve one of Ball's major open problems, see Ball [5], namely the meaningful application of 
polyconvexity to anisotropic materials. In a series of papers, Schröder and Neff [55], Schröder et al. [57], Balzani et al. [6], Schröder et al. [58], Ebbing et al. [13], the concept has been elaborated in detail, with papers from other authors following in due course, see e.g. Itskov et al. [32, Ehret and Itskov [14]. It is, however, clear that polyconvexity (or ellipticity) alone is not sufficient to characterize physically reasonable material response: there exist polyconvex strain energies with unacceptable non-monotone pressure-compression relation. Moreover, the identification of material parameters in the above proposed anisotropic extensions heavily relies on nonlinear optimization strategies after which the physical meaning of the obtained parameters is doubtful: a completely different set of material parameters may equally well fit the available experimental data. This already occurs for the isotropic Ogden-model, see Ogden [49]. The situation for the anisotropic response can only be worse in general. Therefore, the need is to construct strain-energy functions whose possibly few parameters have a clear physical meaning and which are uniquely and easily identified from experiments. At the same time the proposed strain energy should be Legendre-Hadamard elliptic at least in that range of deformation which is typically encountered in the applications. In this paper we numerically explore such a formulation based on the well known logarithmic Hencky strain.

In 1928 Heinrich Hencky [22] proposed the after him named strain-energy function $W_{\mathrm{H}}$ for finite isotropic elasticity. He replaced the small strain tensor $\varepsilon$ in classical linear isotropic energy by the Hencky or logarithmic strain measure $\log \boldsymbol{U}$, with the right stretch tensor $\boldsymbol{U}$. For moderate deformations, this simple function $W_{\mathrm{H}}$ with the two classical Lamé constants is useful for a wide class of materials, see Anand [1; 2] and Bruhns et al. [11]. However, Hencky's energy function is not rank-one convex, i.e. it does not fulfill the well known Legendre-Hadamard, or ellipticity, condition. Bruhns et al. [12] derived necessary and sufficient conditions for ellipticity in terms of principal stretches and computed the largest common ellipticity region. They showed, in the case of positive Lamé constants, that $W_{\mathrm{H}}$ is elliptic whenever every principal stretch is in the range $[0.21162 \ldots, 1.39561 \ldots]$. Furthermore, Hencky's strain-energy automatically satisfies the Baker-Ericksen inequality (Baker and Ericksen [3]) and Hill's inequality (Hill [25; 26]), see Bruhns et al. [12], Ghiba et al. [16].

\subsection{The exponentiated Hencky energy}

The exponentiated Hencky-logarithmic model was recently introduced by Neff et al. [46]. It is induced by the exponentiated Hencky strain energy

$$
\begin{aligned}
W_{\mathrm{eH}}(\boldsymbol{F}) & =\frac{\mu}{k} \exp \left[k\left\|\operatorname{dev}_{n} \log \boldsymbol{U}\right\|^{2}\right]+\frac{\kappa}{2 \hat{k}} \exp \left\{\hat{k}[\operatorname{tr}(\log \boldsymbol{U})]^{2}\right\} \\
& =\frac{\mu}{k} \exp \left[k\left\|\log \frac{\boldsymbol{U}}{(\operatorname{det} \boldsymbol{U})^{1 / n}}\right\|^{2}\right]+\frac{\kappa}{2 \hat{k}} \exp \left[\hat{k}(\log \operatorname{det} \boldsymbol{U})^{2}\right]
\end{aligned}
$$

where $\mu>0$ is the (infinitesimal) shear modulus, $\kappa>0$ is the bulk modulus, $k$ and $\hat{k}$ are additional dimensionless material parameters, $\boldsymbol{U}=\sqrt{\boldsymbol{F}^{T} \boldsymbol{F}}$ is the right stretch tensor corresponding to the deformation gradient $\boldsymbol{F}$, log denotes the principal matrix logarithm on the set of positive definite symmetric matrices, $\operatorname{dev}_{n} \boldsymbol{X}=\boldsymbol{X}-\frac{1}{n} \operatorname{tr} \boldsymbol{X} \mathbf{1}$ and $\left.\|\boldsymbol{X}\|=\sqrt{\operatorname{tr}\left(\boldsymbol{X}^{T} \boldsymbol{X}\right.}\right)$ are the deviatoric part and the Frobenius matrix norm of an $n \times n$ matrix $\boldsymbol{X}$, respectively, and tr denotes the trace operator. 
The exponentiated Hencky energy is based on the so-called volumetric and isochoric logarithmic strain measures $\omega_{\text {iso }}=\left\|\operatorname{dev}_{n} \log \boldsymbol{U}\right\|$ and $\omega_{\mathrm{vol}}=|\operatorname{tr} \log \boldsymbol{U}|=|\log \operatorname{det} \boldsymbol{U}|$, respectively which have recently been given a purely geometric characterization not shared by any other set of isotropic invariants (Neff et al. [48]): consider the general linear group $\mathrm{GL}(n)$ endowed with the canonical left-invariant Riemannian metric $g$, which for $\boldsymbol{A} \in$ $\mathrm{GL}(n)$ and $\boldsymbol{X}, \boldsymbol{Y} \in \mathfrak{g l}(n)=T_{\boldsymbol{A}} \mathrm{GL}(n) \cong \mathbb{R}^{n \times n}$ is given by Martin and Neff [37]

$$
g_{\boldsymbol{A}}(\boldsymbol{X}, \boldsymbol{Y})=\left\langle\boldsymbol{A}^{-1} \boldsymbol{X}, \boldsymbol{A}^{-1} \boldsymbol{Y}\right\rangle
$$

where $\langle\boldsymbol{X}, \boldsymbol{Y}\rangle=\operatorname{tr}\left(\boldsymbol{X} \boldsymbol{Y}^{T}\right)$ is the canonical inner product on the space of real $n \times n$ matrices. Then the logarithmic strain measures are the geodesic distance of the isochoric part $\frac{\boldsymbol{F}}{\operatorname{det} \boldsymbol{F}^{1 / n}}$ and the volumetric part $(\operatorname{det} \boldsymbol{F})^{1 / n} \mathbf{1}$ to $\mathbf{1}$ of the deformation gradient to the special orthogonal group $\mathrm{SO}(n)$, respectively, see Neff et al. [48] (Theorem 3.7):

$$
\begin{aligned}
\left\|\operatorname{dev}_{n} \log \boldsymbol{U}\right\| & =\operatorname{dist}_{\text {geod }}\left(\frac{\boldsymbol{F}}{(\operatorname{det} \boldsymbol{F})^{1 / n}}, \mathrm{SO}(n)\right), \\
|\log \operatorname{det} \boldsymbol{F}| & =\operatorname{dist}_{\text {geod }}\left((\operatorname{det} \boldsymbol{F})^{1 / n} \cdot \mathbf{1}, \mathrm{SO}(n)\right) .
\end{aligned}
$$

These two quantities are thereby identified as the "natural" measures of strain in any deformation, an observation which strongly suggests that an idealized elastic strain energy function may depend on these quantities alone $1^{1}$ An important example of such an energy function is the classical quadratic Hencky energy

$$
\begin{aligned}
W_{\mathrm{H}}(\boldsymbol{F}) & =\mu\left\|\operatorname{dev}_{n} \log \boldsymbol{U}\right\|^{2}+\frac{\kappa}{2}[\operatorname{tr}(\log \boldsymbol{U})]^{2} \\
& =\mu\left\|\log \frac{\boldsymbol{U}}{(\operatorname{det} \boldsymbol{U})^{1 / n}}\right\|^{2}+\frac{\kappa}{2}(\log \operatorname{det} \boldsymbol{U})^{2},
\end{aligned}
$$

which was introduced by Heinrich Hencky in 1929 (Hencky [23], Neff et al. [45]). While the elasticity model induced by the Hencky energy is in very good agreement with experimental observations for up to moderate strains for a large number of materials Neff et al. [46], Anand [1, there are some major shortcomings of this model. For example, the qualitative behavior of materials under very large deformations is not modeled accurately, and since the energy function is neither polyconvex nor quasiconvex or rank-one convex (Neff [41], Ghiba et al. [16]), no known methods are available to ensure the existence of energy minimizers for general boundary value problems. Moreover, the pressurecompression relation is not monotone.

In order to alleviate some of these shortcomings, Neff et al. introduced the exponentiated Hencky energy $W_{\mathrm{eH}}$ in a series of articles Neff et al. [46; 47], Neff and Ghiba [42], Ghiba et al. [17]. This energy function closely approximates the classical quadratic Hencky energy for small deformations, but aims to provide a more accurate model for large deformations as well as an improvement in terms of common constitutive requirements; for example, $W_{\mathrm{eH}}$ is polyconvex in the two-dimensional case Neff et al. [47], and in the three-dimensional case the rank-one convexity domain contains the extremely large set $\left\{\boldsymbol{F} \in \mathrm{GL}^{+}(3) \mid\left\|\operatorname{dev}_{3} \log \boldsymbol{U}\right\| \leq 6\right\}$. Moreover, the induced mapping

\footnotetext{
${ }^{1}$ Note that not every objective and isotropic energy function can be expressed in terms of the logarithmic strain measures alone, see Neff et al. [48], whereas every such energy can be expressed in terms of the logarithmic strain tensor $\log \boldsymbol{U}$.
} 
$\boldsymbol{B} \mapsto \boldsymbol{\sigma}$ of the Finger tensor $\boldsymbol{B}=\boldsymbol{F} \boldsymbol{F}^{T}$ to the Cauchy stress tensor $\boldsymbol{\sigma}$ is invertible Neff and Mihai [43], Mihai and Neff [38; 39], Jog and Patil [34, as is the case for suitable variants of the Neo-Hooke and Mooney-Rivlin energies for slightly compressible materials like rubber.

The low number of additional material parameters in the exponentiated Hencky model also suggests that a good material fitting could be possible even without extensive experimental measurements. Additionally, the exponentiated Hencky energy allows for the modeling of a zero apparent Poisson's modulus $\nu=\frac{3 \kappa-2 \mu}{2(3 \kappa+\mu)}$ in the finite strain regime: if the additional parameters $k, \hat{k}$ are chosen such that $k=\frac{2}{3} \hat{k}$, then $W_{\mathrm{eH}}$ can be written as

$$
\frac{1}{2 k}\left(\frac{E}{1+\nu} \exp \left[k\left\|\operatorname{dev}_{n} \log \boldsymbol{U}\right\|^{2}\right]+\frac{E}{2(1-2 \nu)} \exp \left[\frac{2}{3} k(\log \operatorname{det} \boldsymbol{U})^{2}\right]\right),
$$

where $E=\frac{9 \kappa \mu}{3 \kappa+\mu}$ is Young's modulus, and for $\nu=0$ we obtain a model with zero lateral contraction under finite strains Neff et al. [46].

A variant of the exponentiated Hencky energy has previously been applied to so-called tire derived materials and was found to be in good agreement with experimental data, see Montella et al. [40]. In particular, the highly nonlinear equation of state (EOS) relating pressure to purely volumetric deformations has been captured extraordinarily well. The extra appearing non-dimensional parameters $k$ and $\hat{k}$ have an intuitive meaning: larger $k, \hat{k}$ lead to monotonically increased strain hardening. In principal these parameters can be fitted independent of the shear and bulk modulus. Next, we extend the exponential Hencky energy to the anisotropic case.

\section{Theoretical framework}

\subsection{Kinematics}

For a better overview, the continuum-mechanical kinematic and constitutive quantities are listed in Table 1 . Let $\mathcal{B} \subset \mathbb{R}^{3}$ be the body of interest in the reference placement, parametrized in $\boldsymbol{X}$, and let $\mathcal{S}$ be the body in the current placement, parametrized in $\boldsymbol{x}$. The boundary $\partial \mathcal{B}$ of $\mathcal{B}$ is decomposed in $\partial \mathcal{B}_{u}$ and $\partial \mathcal{B}_{t}$ with $\partial \mathcal{B}_{u} \cup \partial \mathcal{B}_{t}=\partial \mathcal{B}$ and $\partial \mathcal{B}_{u} \cap \partial \mathcal{B}_{t}=\emptyset$. The nonlinear deformation map is given by $\boldsymbol{x}=\boldsymbol{\varphi}(\boldsymbol{X})$. As basic kinematical quantities we define the deformation gradient and the right Cauchy-Green tensor

$$
\boldsymbol{F}=\operatorname{Grad} \boldsymbol{\varphi}(\boldsymbol{X}) \quad \text { and } \quad \boldsymbol{C}=\boldsymbol{F}^{T} \boldsymbol{F}=\boldsymbol{U}^{2},
$$

respectively. Here, $\mathbf{1}$ denotes the second-order identity tensor. The Jacobian of the deformation gradient has to satisfy $J:=\operatorname{det} \boldsymbol{F}>0$. The deformation gradient may be split into

$$
\boldsymbol{F}=\boldsymbol{R} \boldsymbol{U}=\boldsymbol{V} \boldsymbol{R}
$$

where $\boldsymbol{R} \in \mathrm{SO}(3)$ denotes a pure rotation tensor and $\boldsymbol{U}$ and $\boldsymbol{V}$ are the right and left stretch tensors, respectively. In order to fulfill the principle of material frame indifference a priori, we formulate the generic free energy function $\psi$ in terms of the right CauchyGreen tensor, i.e. $\psi=\psi(\boldsymbol{C})$. In spectral decomposition the right Cauchy-Green tensor $\boldsymbol{C}$ and the left Cauchy-Green tensor $\boldsymbol{B}$ may be written as

$$
\boldsymbol{C}=\sum_{k=1}^{3} \widehat{\lambda}_{k} \boldsymbol{N}^{k} \otimes \boldsymbol{N}^{k}, \quad \boldsymbol{B}=\sum_{k=1}^{3} \widehat{\lambda}_{k} \boldsymbol{n}^{k} \otimes \boldsymbol{n}^{k}
$$


Table 1: Kinematic and constitutive quantities.

\begin{tabular}{ll}
\hline Symbol & Continuum mechanical description \\
\hline $\boldsymbol{u}$ & displacement vector \\
$\boldsymbol{F}=\mathbf{1}+$ Grad $\boldsymbol{u}$ & deformation gradient \\
$\boldsymbol{C}=\boldsymbol{F}^{T} \boldsymbol{F}$ & right Cauchy-Green tensor \\
$\boldsymbol{B}=\boldsymbol{F} \boldsymbol{F}^{T}$ & left Cauchy-Green tensor \\
$\boldsymbol{U}=\sqrt{\boldsymbol{F}^{T} \boldsymbol{F}}$ & right stretch tensor \\
$\boldsymbol{V}=\sqrt{\boldsymbol{F} \boldsymbol{F}^{T}}$ & left stretch tensor \\
$\log \boldsymbol{U}$ & right Hencky strain tensor \\
$\log \boldsymbol{V}$ & left Hencky strain tensor \\
\hline$\psi$ & generic elastic energy \\
$W_{\mathrm{H}}$ & isotropic Hencky energy \\
$W_{\mathrm{eH}}$ & exponentiated Hencky energy \\
\hline $\boldsymbol{S}=2 \partial_{\boldsymbol{C}} \psi(\boldsymbol{C})$ & second Piola-Kirchoff stress tensor \\
$\boldsymbol{\tau}=\partial_{\log \boldsymbol{V}} \psi(\log \boldsymbol{V})$ & Kirchoff stress tensor (see Vallée [61] $)$ \\
$\boldsymbol{\sigma}=(\operatorname{det} \boldsymbol{F})^{-1} \boldsymbol{\tau}$ & Cauchy stress tensor \\
\hline
\end{tabular}

where $\widehat{\lambda}_{k}$ denote the eigenvalues of $\boldsymbol{C}$ and $\boldsymbol{B}$. The eigenvectors are expressed through $\boldsymbol{N}^{k}$ and $\boldsymbol{n}^{k}$ associated to $\boldsymbol{C}$ and $\boldsymbol{B}$, respectively. Therefore, we obtain the tensor functions

$$
\begin{gathered}
\boldsymbol{U}=\sqrt{\boldsymbol{C}}=\sum_{k=1}^{3} \sqrt{\widehat{\lambda}_{k}} \boldsymbol{N}^{k} \otimes \boldsymbol{N}^{k}=\sum_{k=1}^{3} \lambda_{k} \boldsymbol{N}^{k} \otimes \boldsymbol{N}^{k} \\
\boldsymbol{V}=\sqrt{\boldsymbol{B}}=\sum_{k=1}^{3} \sqrt{\widehat{\lambda}_{k}} \boldsymbol{n}^{k} \otimes \boldsymbol{n}^{k}=\sum_{k=1}^{3} \lambda_{k} \boldsymbol{n}^{k} \otimes \boldsymbol{n}^{k} \\
\log \boldsymbol{U}=\log (\sqrt{\boldsymbol{C}})=\frac{1}{2} \log \boldsymbol{C} \stackrel{!}{=} \sum_{k=1}^{3} \frac{1}{2} \log \widehat{\lambda}_{k} \boldsymbol{N}^{k} \otimes \boldsymbol{N}^{k} \stackrel{!}{=} \sum_{k=1}^{3} \log \lambda_{k} \boldsymbol{N}^{k} \otimes \boldsymbol{N}^{k} \\
\log \boldsymbol{V}=\log (\sqrt{\boldsymbol{B}})=\frac{1}{2} \log \boldsymbol{B} \stackrel{!}{=} \sum_{k=1}^{3} \frac{1}{2} \log \widehat{\lambda}_{k} \boldsymbol{n}^{k} \otimes \boldsymbol{n}^{k} \stackrel{!}{=} \sum_{k=1}^{3} \log \lambda_{k} \boldsymbol{n}^{k} \otimes \boldsymbol{n}^{k}
\end{gathered}
$$

where $\lambda_{k}$ denote the eigenvalues of $\boldsymbol{U}$ and $\boldsymbol{V}$. The tensor $\log \boldsymbol{U}$ is called right Hencky strain tensor.

\subsection{Stress measures}

Let $\partial_{\log \boldsymbol{U}} \widehat{\psi}(\log \boldsymbol{U})$ be the stress measure work conjugate to $\log \boldsymbol{U}=\frac{1}{2} \log \boldsymbol{C}$, then the transformation rule for the second Piola-Kirchhoff stress tensor $\boldsymbol{S}$ is given by

$$
\begin{aligned}
& \boldsymbol{S}=2 \frac{\partial \widehat{\psi}(\log \boldsymbol{U})}{\partial \boldsymbol{C}}=2 \frac{\partial \widehat{\psi}(\log \boldsymbol{U})}{\partial \log \boldsymbol{U}}: \frac{\partial \log \boldsymbol{U}}{\partial \boldsymbol{C}}=\frac{\partial \widehat{\psi}(\log \boldsymbol{U})}{\partial \log \boldsymbol{U}}: \mathbb{P}_{\mathrm{H}} \\
& \text { with } \mathbb{P}_{\mathrm{H}}=2 \frac{\partial \log \boldsymbol{U}}{\partial \boldsymbol{C}}
\end{aligned}
$$


The fourth-order tensor $\mathbb{P}_{\mathrm{H}}$ can only be derived with the help of the spectral decomposition and yields

$$
\begin{aligned}
\mathbb{P}_{\mathrm{H}}= & \sum_{k=1}^{3} \sum_{j=1}^{3} P_{k k j j} \boldsymbol{N}^{k} \otimes \boldsymbol{N}^{k} \otimes \boldsymbol{N}^{j} \otimes \boldsymbol{N}^{j} \\
& +2 \sum_{k=1}^{3} \sum_{k \neq j}^{3} P_{k j k j}\left(\boldsymbol{N}^{k} \otimes \boldsymbol{N}^{j}\right) \otimes\left(\boldsymbol{N}^{k} \otimes \boldsymbol{N}^{j}+\boldsymbol{N}^{j} \otimes \boldsymbol{N}^{k}\right) \\
P_{k k j j}= & \delta_{k j} \widehat{\lambda}_{k}^{-1} \\
P_{k j k j}= & \begin{cases}\frac{\frac{1}{2} \log \widehat{\lambda}_{k}-\frac{1}{2} \log \widehat{\lambda}_{j}}{\widehat{\lambda}_{k}-\widehat{\lambda}_{j}} & \text { for } \widehat{\lambda}_{k} \neq \widehat{\lambda}_{j} \\
\lim _{\widehat{\lambda}_{k} \rightarrow \widehat{\lambda}_{j}} \frac{\frac{1}{2} \log \widehat{\lambda}_{k}-\frac{1}{2} \log \widehat{\lambda}_{j}}{\widehat{\lambda}_{k}-\widehat{\lambda}_{j}}=: \partial_{\widehat{\lambda}_{k}}\left(\frac{1}{2} \log \widehat{\lambda}_{k}\right)=\left(2 \widehat{\lambda}_{k}\right)^{-1} & \text { for } \widehat{\lambda}_{k}=\widehat{\lambda}_{j}\end{cases}
\end{aligned}
$$

see also Ogden [50] and Simo [59]. The first part of $\mathbb{P}_{\mathrm{H}}$ is related to the derivative of the eigenvalues of $\log \boldsymbol{U}$ with respect to $\boldsymbol{C}$, while the second part is related to $\partial_{\boldsymbol{C}}\left(\boldsymbol{N}^{k} \otimes \boldsymbol{N}^{k}\right)$. In the isotropic case the following relations regarding the Kirchoff stress

$$
\begin{aligned}
\boldsymbol{\tau} & =\frac{\partial \widetilde{\psi}(\log \boldsymbol{V})}{\partial \log \boldsymbol{V}}=\boldsymbol{R} \frac{\partial \widehat{\psi}(\log \boldsymbol{U})}{\partial \log \boldsymbol{U}} \boldsymbol{R}^{T}=\boldsymbol{R} \frac{\partial \psi^{\#}(\boldsymbol{U})}{\partial \boldsymbol{U}} \boldsymbol{U} \boldsymbol{R}^{T} \\
& =2 \boldsymbol{F} \frac{\partial \bar{\psi}(\boldsymbol{C})}{\partial \boldsymbol{C}} \boldsymbol{F}^{T}=2 \boldsymbol{B} \frac{\partial \psi^{+}(\boldsymbol{B})}{\partial \boldsymbol{B}}=\frac{\partial \psi(\boldsymbol{F})}{\partial \boldsymbol{F}} \boldsymbol{F}^{T}
\end{aligned}
$$

hold true. But if anisotropic behavior is considered only the relations

$$
\boldsymbol{\tau}=2 \boldsymbol{F} \frac{\partial \bar{\psi}(\boldsymbol{C})}{\partial \boldsymbol{C}} \boldsymbol{F}^{T}=\frac{\partial \psi(\boldsymbol{F})}{\partial \boldsymbol{F}} \boldsymbol{F}^{T}
$$

remain valid. For the derivation of the above mentioned relations the reader is referred to the appendix. The expression $\boldsymbol{T}_{\text {Biot }}=\partial_{\boldsymbol{U}} \psi^{\#}(\boldsymbol{U})$ is also known as Biot-stress. For the linearization of the weak form, we need the tangent moduli

$$
\mathbb{C}=4 \frac{\partial^{2} \widehat{\psi}(\log \boldsymbol{U})}{\partial \boldsymbol{C} \partial \boldsymbol{C}}=\mathbb{P}_{\mathrm{H}}: \mathbb{C}^{\mathrm{H}}: \mathbb{P}_{\mathrm{H}}+\frac{\partial \widehat{\psi}(\log \boldsymbol{U})}{\partial \log \boldsymbol{U}}: \mathbb{K}
$$

with

$$
\mathbb{C}^{\mathrm{H}}=\frac{\partial^{2} \hat{\psi}(\log \boldsymbol{U})}{\partial \log \boldsymbol{U} \partial \log \boldsymbol{U}} \quad \text { and } \quad \mathbb{K}=2 \frac{\partial \mathbb{P}_{\mathrm{H}}}{\partial \boldsymbol{C}}=4 \frac{\partial^{2} \log \boldsymbol{U}}{\partial \boldsymbol{C} \partial \boldsymbol{C}} .
$$

The multiplicative volumetric isochoric decomposition of the deformation gradient

$$
\widetilde{\boldsymbol{F}}=J^{-1 / 3} \boldsymbol{F} \quad \text { and } \quad \widetilde{\boldsymbol{C}}=J^{-2 / 3} \boldsymbol{C}
$$

was first proposed by Hans Richter [52], see also Flory [15]. In doing so we can express the volumetric Hencky strain tensor with help of an additive split according to

$$
\log \boldsymbol{U}=\operatorname{dev}(\log \boldsymbol{U})+\frac{1}{3} \operatorname{tr}(\log \boldsymbol{U}) \mathbf{1}, \quad \text { with } \quad \operatorname{tr}(\operatorname{dev} \log \boldsymbol{U})=0 .
$$

For the numerical treatment of an energy function $\psi^{*}(\operatorname{dev} \log \boldsymbol{U})$ we need the derivative

$$
\frac{\partial \operatorname{dev} \log \boldsymbol{U}}{\partial \log \boldsymbol{U}}=\mathbf{1} \otimes \mathbf{1}-\frac{1}{3} \mathbf{1} \otimes \mathbf{1}=\mathbb{P},
$$


where $\boldsymbol{\otimes}$ denotes the Kronecker product of second-order tensors. Let $\boldsymbol{G}$ and $\boldsymbol{H}$ denote two second-order tensors and $\boldsymbol{g}$ and $\boldsymbol{h}$ two first-order tensors, then the operator is defined by $(\boldsymbol{G} \otimes \boldsymbol{H}):(\boldsymbol{g} \otimes \boldsymbol{h})=(\boldsymbol{G} \boldsymbol{g}) \otimes(\boldsymbol{H} \boldsymbol{h})$. Formulating a strain energy in dev $\log \boldsymbol{U}$, before projecting the stress tensor and tangent moduli on $\boldsymbol{C}$ we first need to project them on the Hencky strain $\log \boldsymbol{U}$. Therefore, we define

$$
\frac{\partial \psi^{*}(\operatorname{dev} \log \boldsymbol{U})}{\partial \log \boldsymbol{U}}=\frac{\partial \psi^{*}(\operatorname{dev} \log \boldsymbol{U})}{\partial \operatorname{dev} \log \boldsymbol{U}}: \frac{\partial \operatorname{dev} \log \boldsymbol{U}}{\partial \log \boldsymbol{U}}=\frac{\partial \psi^{*}(\operatorname{dev} \log \boldsymbol{U})}{\partial \operatorname{dev} \log \boldsymbol{U}}: \mathbb{P},
$$

and for the linearization of the weak form it follows

$$
\mathbb{C}^{\mathrm{H}}=\frac{\partial^{2} \psi^{*}(\operatorname{dev} \log \boldsymbol{U})}{\partial \log \boldsymbol{U} \partial \log \boldsymbol{U}}=\mathbb{P}: \widetilde{\mathbb{C}}^{\mathrm{H}}: \mathbb{P},
$$

with

$$
\widetilde{\mathbb{C}}^{H}=\frac{\partial^{2} \psi^{*}(\operatorname{dev} \log \boldsymbol{U})}{\partial \operatorname{dev} \log \boldsymbol{U} \partial \operatorname{dev} \log \boldsymbol{U}} .
$$

The corresponding tensors are to be inserted in Eq. (10) and Eq. (14).

\subsection{Isotropic and anisotropic invariants}

The principal isotropic invariants of the right Cauchy-Green tensor $\boldsymbol{C}$ are given by

$$
\begin{aligned}
& I_{1}^{\mathrm{C}}=\operatorname{tr}[\boldsymbol{C}]=\|\boldsymbol{F}\|^{2}, \quad I_{2}^{\mathrm{C}}=\operatorname{tr}[\operatorname{Cof} \boldsymbol{C}]=\|\operatorname{Cof} \boldsymbol{F}\|^{2} \quad \text { and } \\
& I_{3}^{\mathrm{C}}=\operatorname{det} \boldsymbol{C}=(\operatorname{det} \boldsymbol{F})^{2}=J^{2} .
\end{aligned}
$$

Further, we introduce the basic invariants of the Hencky strain tensor $\log \boldsymbol{U}$

$$
\begin{aligned}
& J_{1}^{\mathrm{H}}=\log (\operatorname{det} \boldsymbol{U})=\operatorname{tr}(\log \boldsymbol{U}), \quad J_{2}^{\mathrm{H}}=\|\log \boldsymbol{U}\|^{2}=\operatorname{tr}\left[(\log \boldsymbol{U})^{2}\right] \quad \text { and } \\
& J_{3}^{\mathrm{H}}=\operatorname{tr}\left[(\log \boldsymbol{U})^{3}\right]
\end{aligned}
$$

already used by Richter [52]. Let $\boldsymbol{A}$, with $\|\boldsymbol{A}\|=1$, be the preferred direction of the transversely isotropic material, then the material symmetry group is defined by

$$
\mathcal{G}_{t i}:=\{ \pm \mathbf{1} ; \boldsymbol{Q}(\alpha, \boldsymbol{A}) \mid 0<\alpha<2 \pi\}
$$

where $\boldsymbol{Q}(\alpha, \boldsymbol{A})$ are all rotations along the $\boldsymbol{A}$-axis. The structural tensor $\boldsymbol{M}$ whose invariance group preserves the material symmetry group $\mathcal{G}_{t i}$ is given by the rank-one tensor

$$
\boldsymbol{M}=\boldsymbol{A} \otimes \boldsymbol{A}
$$

see Boehler [8] and Boehler [9] regarding the concept of structural tensors. Based on the structural tensor we define the mixed invariants

$$
\begin{array}{ll}
I_{4}^{\mathrm{C}^{i}}=\left\langle\boldsymbol{C}^{i}, \boldsymbol{M}\right\rangle, & J_{5}^{\mathrm{C}}=\langle\operatorname{Cof} \boldsymbol{C}, \boldsymbol{M}\rangle, \\
I_{4}^{\mathrm{H}^{i}}=\left\langle(\log \boldsymbol{U})^{i}, \boldsymbol{M}\right\rangle, & J_{5}^{\mathrm{H}}=\langle\log (\operatorname{Cof} \boldsymbol{U}), \boldsymbol{M}\rangle,
\end{array}
$$

where $i \in \mathbb{N}, i>0$, denotes an exponent. Note that the cofactor $\operatorname{Cof}(\log \boldsymbol{U})$ has no physical meaning and that for $\boldsymbol{U} \in \operatorname{Sym}^{+}(3)$, Cof $\boldsymbol{U}$ is also positive definite. Because of 
that we instead consider the logarithmic cofactor function

$$
\begin{aligned}
\log (\operatorname{Cof} \boldsymbol{U})= & \log \left[(\operatorname{det} \boldsymbol{U}) \boldsymbol{U}^{-1}\right]=\sum_{k=1}^{3} \log \left(\frac{\operatorname{det} \sqrt{\boldsymbol{C}}}{\widehat{\lambda}_{k}^{1 / 2}}\right) \boldsymbol{N}^{k} \otimes \boldsymbol{N}^{k} \\
= & \sum_{k=1}^{3}\left[\log (\operatorname{det} \sqrt{\boldsymbol{C}})-\frac{1}{2} \log \widehat{\lambda}_{k}\right] \boldsymbol{N}^{k} \otimes \boldsymbol{N}^{k} \\
= & \left(\frac{1}{2} \log \widehat{\lambda}_{2}+\frac{1}{2} \log \widehat{\lambda}_{3}\right) \boldsymbol{N}^{1} \otimes \boldsymbol{N}^{1}+\left(\frac{1}{2} \log \widehat{\lambda}_{1}+\frac{1}{2} \log \widehat{\lambda}_{3}\right) \boldsymbol{N}^{2} \otimes \boldsymbol{N}^{2} \\
& +\left(\frac{1}{2} \log \widehat{\lambda}_{1}+\frac{1}{2} \log \widehat{\lambda}_{2}\right) \boldsymbol{N}^{3} \otimes \boldsymbol{N}^{3} \\
= & \operatorname{tr}(\log \boldsymbol{U}) \mathbf{1}-\log \boldsymbol{U}
\end{aligned}
$$

and finally we observe the following

$$
J_{5}^{\mathrm{H}}=\langle\log (\operatorname{Cof} \boldsymbol{U}), \boldsymbol{M}\rangle=\operatorname{tr}(\log \boldsymbol{U}) \underbrace{\langle\boldsymbol{M}, \mathbf{1}\rangle}_{1}-\langle\log \boldsymbol{U}, \boldsymbol{M}\rangle=J_{1}^{\mathrm{H}}-I_{4}^{\mathrm{H}^{1}} .
$$

\section{$3 \quad$ Isotropic strain energy functions}

\subsection{Isotropic Hencky Energy}

The isotropic Hencky energy was introduced in Hencky [23]. It measures the geodesic distance of the deformation gradient to the special orthogonal group $\mathrm{SO}(n)$, as it was discovered in Neff et al. [44]. The Hencky strain energy

$$
W_{\mathrm{H}}(\log \boldsymbol{U})=\mu\|\operatorname{dev} \log \boldsymbol{U}\|^{2}+\frac{\kappa}{2}[\operatorname{tr}(\log \boldsymbol{U})]^{2}=\mu\|\log \boldsymbol{U}\|^{2}+\frac{\lambda}{2}[\operatorname{tr}(\log \boldsymbol{U})]^{2}
$$

can be reformulated in principal logarithmic strains

$$
\begin{aligned}
W_{\mathrm{H}}(\log \boldsymbol{U})= & \mu\left[\left(\frac{1}{2} \log \widehat{\lambda}_{1}\right)^{2}+\left(\frac{1}{2} \log \widehat{\lambda}_{2}\right)^{2}+\left(\frac{1}{2} \log \widehat{\lambda}_{3}\right)^{2}\right] \\
& +\frac{\lambda}{2}\left[\frac{1}{2} \log \widehat{\lambda}_{1}+\frac{1}{2} \log \widehat{\lambda}_{2}+\frac{1}{2} \log \widehat{\lambda}_{3}\right]^{2}
\end{aligned}
$$

based on the eigenvalues $\hat{\lambda}$ of $\boldsymbol{C}$, where the Lamé parameters $\lambda$ and $\mu$ as well as the bulk modulus $\kappa$ are used. Note that $\kappa=\frac{3 \lambda+2 \mu}{3}$ and

$$
\begin{aligned}
\|\operatorname{dev}(\log \boldsymbol{U})\|^{2}= & \frac{1}{3}\left[\left(\log \sqrt{\frac{\widehat{\lambda}_{1}}{\widehat{\lambda}_{2}}}\right)^{2}+\left(\log \sqrt{\frac{\widehat{\lambda}_{1}}{\widehat{\lambda}_{3}}}\right)^{2}+\left(\log \sqrt{\frac{\widehat{\lambda}_{2}}{\widehat{\lambda}_{3}}}\right)^{2}\right] \\
= & \frac{2}{3}\left[\left(\frac{1}{2} \log \widehat{\lambda}_{1}\right)^{2}+\left(\frac{1}{2} \log \widehat{\lambda}_{2}\right)^{2}+\left(\frac{1}{2} \log \widehat{\lambda}_{3}\right)^{2}\right] \\
& -\frac{2}{3}\left[\frac{1}{4} \log \widehat{\lambda}_{1} \log \widehat{\lambda}_{2}+\frac{1}{4} \log \widehat{\lambda}_{1} \log \widehat{\lambda}_{3}+\frac{1}{4} \log \widehat{\lambda}_{2} \log \widehat{\lambda}_{3}\right] .
\end{aligned}
$$


The function $W_{\mathrm{H}}$ is not polyconvex, not quasiconvex, not coercive and not rank-oneelliptic, even for every admissible deformation state, see Neff et al. [46]. However, it holds that $W_{\mathrm{H}}(\boldsymbol{F})=W_{\mathrm{H}}\left(\boldsymbol{F}^{-1}\right)$. The first and second derivative with respect to the Hencky strain yield

$$
\begin{aligned}
\frac{\partial W_{\mathrm{H}}}{\partial \log \boldsymbol{U}} & =2 \mu \operatorname{dev}(\log \boldsymbol{U})+\kappa \operatorname{tr}(\log \boldsymbol{U}) \mathbf{1}, \\
\mathbb{C}^{\mathrm{H}} & =\frac{\partial^{2} W_{\mathrm{H}}}{\partial \log \boldsymbol{U} \partial \log \boldsymbol{U}}=2 \mu \mathbb{P}+\kappa \mathbf{1} \otimes \mathbf{1} .
\end{aligned}
$$

In the reference configuration with $\boldsymbol{C}=\mathbf{1}$ the final material tangent $\mathbb{C}$, in Voigt-notation $\bigsqcup^{2}$ denoted as $\mathbb{C}^{V}$, according to Eq. (14) simplifies to

$$
\left.\mathbb{C}^{\mathrm{V}}\right|_{\boldsymbol{C = 1}}=\left.4 \frac{\partial^{2} W_{\mathrm{H}}}{\partial \boldsymbol{C} \partial \boldsymbol{C}}\right|_{\boldsymbol{C = 1}}=\left(\begin{array}{cccccc}
\kappa+\frac{4}{3} \mu & \kappa-\frac{2}{3} \mu & \kappa-\frac{2}{3} \mu & 0 & 0 & 0 \\
\kappa-\frac{2}{3} \mu & \kappa+\frac{4}{3} \mu & \kappa-\frac{2}{3} \mu & 0 & 0 & 0 \\
\kappa-\frac{2}{3} \mu & \kappa-\frac{2}{3} \mu & \kappa+\frac{4}{3} \mu & 0 & 0 & 0 \\
0 & 0 & 0 & \mu & 0 & 0 \\
0 & 0 & 0 & 0 & \mu & 0 \\
0 & 0 & 0 & 0 & 0 & \mu
\end{array}\right)
$$

\subsection{Exponentiated Hencky energy}

The exponentiated Hencky energy

$$
\begin{aligned}
W_{\mathrm{eH}} & =\frac{\mu}{k} \exp \left[k\|\operatorname{dev}(\log \boldsymbol{U})\|^{2}\right]+\frac{\kappa}{2 \hat{k}} \exp \left[\hat{k}(\operatorname{tr} \log \boldsymbol{U})^{2}\right] \\
& =\frac{\mu}{k} \exp \left[k\left\|\operatorname{dev}\left(\frac{1}{2} \log \boldsymbol{C}\right)\right\|^{2}\right]+\frac{\kappa}{2 \hat{k}} \exp \left[\hat{k}\left\langle\mathbf{1}, \frac{1}{2} \log \boldsymbol{C}\right\rangle^{2}\right], \quad k>\frac{1}{3}, \hat{k}>\frac{1}{8}
\end{aligned}
$$

was introduced and described in Neff et al. [46]. It is still volumetric-isochoric decoupled and polyconvex in 2D if $k>\frac{1}{3}$ and $\hat{k}>\frac{1}{8}$, cf. Neff et al. [47]. Rank-one convexity is not preserved in 3D, see Neff et al. [46]. However, numerical calculations show that the ellipticity domain contains the extremely large set $\left\{\boldsymbol{F} \in \mathrm{GL}^{+}(3) \mid\left\|\operatorname{dev}_{3} \log \boldsymbol{U}\right\| \leq 6\right\}$. In the small strain regime for principal stretches $\lambda_{i} \in(0.7,1.4)$ it approximates the aforementioned isotropic Hencky energy quite well.

Reformulation in terms of the Lamé parameters $\mu$ and $\lambda$ yields

$$
W_{\mathrm{eH}}=\frac{\mu}{k} \exp \left[k\left\|\operatorname{dev}\left(\frac{1}{2} \log \boldsymbol{C}\right)\right\|^{2}\right]+\frac{(3 \lambda+2 \mu) / 3}{2 \hat{k}} \exp \left[\hat{k}\left\langle\mathbf{1}, \frac{1}{2} \log \boldsymbol{C}\right\rangle^{2}\right] .
$$

The derivatives with respect to the Hencky strain yield

$$
\begin{aligned}
\frac{\partial W_{\mathrm{eH}}}{\partial \log \boldsymbol{U}}= & 2 \mu \exp \left[k\|\operatorname{dev}(\log \boldsymbol{U})\|^{2}\right] \operatorname{dev}(\log \boldsymbol{U}) \\
& +\kappa \exp \left[\hat{k}(\operatorname{tr} \log \boldsymbol{U})^{2}\right](\operatorname{tr} \log \boldsymbol{U}) \mathbf{1},
\end{aligned}
$$

\footnotetext{
${ }^{2}$ In the contracted notation the tensorial indices are allocated to the matrix indexes as follows $\{11,22,33,12,23,13\} \rightarrow\{1,2,3,4,5,6\}$.
} 


$$
\begin{aligned}
\mathbb{C}^{\mathrm{H}}=\frac{\partial^{2} W_{\mathrm{eH}}}{\partial \log \boldsymbol{U} \partial \log \boldsymbol{U}}= & 4 \mu \exp \left[k\left\|\operatorname{dev}\left(\frac{1}{2} \log \boldsymbol{C}\right)\right\|^{2}\right] \operatorname{dev}\left(\frac{1}{2} \log \boldsymbol{C}\right) \otimes \operatorname{dev}\left(\frac{1}{2} \log \boldsymbol{C}\right) \\
& +2 \mu \exp \left[k\left\|\operatorname{dev}\left(\frac{1}{2} \log \boldsymbol{C}\right)\right\|^{2}\right] \mathbb{P} \\
& +\kappa \exp \left[\hat{k}\left\langle\mathbf{1}, \frac{1}{2} \log \boldsymbol{C}\right\rangle^{2}\right] \mathbf{1} \otimes \mathbf{1} \\
& +2 \kappa \hat{k} \exp \left[\hat{k}\left\langle\mathbf{1}, \frac{1}{2} \log \boldsymbol{C}\right\rangle^{2}\right]\left\langle\mathbf{1}, \frac{1}{2} \log \boldsymbol{C}\right\rangle^{2} \mathbf{1} \otimes \mathbf{1} .
\end{aligned}
$$

In the reference configuration with $\boldsymbol{C}=\mathbf{1}$ and $\log \boldsymbol{U}=\mathbf{0}$ the above equation simplifies to

$$
\left.\frac{\partial^{2} W_{\mathrm{eH}}}{\partial^{2} \log \boldsymbol{U}}\right|_{\boldsymbol{C}=\mathbf{1}}=2 \mu \mathbb{P}+\kappa \mathbf{1} \otimes \mathbf{1}
$$

and the final tangent according to Eq. (14) becomes

$$
\left.\mathbb{C}^{\mathrm{V}}\right|_{\boldsymbol{C = 1}}=\left.4 \frac{\partial^{2} W_{\mathrm{eH}}}{\partial \boldsymbol{C} \partial \boldsymbol{C}}\right|_{\boldsymbol{C = 1}}=\left(\begin{array}{cccccc}
\kappa+\frac{4}{3} \mu & \kappa-\frac{2}{3} \mu & \kappa-\frac{2}{3} \mu & 0 & 0 & 0 \\
\kappa-\frac{2}{3} \mu & \kappa+\frac{4}{3} \mu & \kappa-\frac{2}{3} \mu & 0 & 0 & 0 \\
\kappa-\frac{2}{3} \mu & \kappa-\frac{2}{3} \mu & \kappa+\frac{4}{3} \mu & 0 & 0 & 0 \\
0 & 0 & 0 & \mu & 0 & 0 \\
0 & 0 & 0 & 0 & \mu & 0 \\
0 & 0 & 0 & 0 & 0 & \mu
\end{array}\right)
$$

which is identical to the elasticity tensor of $W_{H}$, provided in Eq. (34).

\section{Anisotropic extension}

\subsection{Transverse isotropic Hencky and exponentiated Hencky models}

In a first step we aim to investigate a strain energy function $W_{1}\left(J_{1}^{\mathrm{H}},\|\operatorname{dev} \log \boldsymbol{U}\|, I_{4}^{\mathrm{H}^{1}}, I_{4}^{\mathrm{H}^{2}}\right)$ which basically extends the classical Hencky-strain energy by the basic mixed invariants, introduced in chapter 2.3 ;

$$
\begin{aligned}
W_{1}(\log \boldsymbol{U})= & \mu_{\mathrm{T}}\|\operatorname{dev} \log \boldsymbol{U}\|^{2}+\frac{\kappa}{2}[\operatorname{tr}(\log \boldsymbol{U})]^{2}+\alpha\langle\log \boldsymbol{U}, \boldsymbol{M}\rangle(\operatorname{tr} \log \boldsymbol{U}) \\
& +2\left(\mu_{\mathrm{L}}-\mu_{\mathrm{T}}\right)\left\langle\boldsymbol{M},(\log \boldsymbol{U})^{2}\right\rangle+\frac{1}{2} \beta\langle\boldsymbol{M}, \log \boldsymbol{U}\rangle^{2} .
\end{aligned}
$$

The parameters are chosen in analogy to Spencer [60]. Here, $\mu_{T}$ and $\mu_{L}$ are associated to the shear moduli in the transverse isotropy plane and perpendicular to that, $\kappa$ is associated to the bulk modulus, $\beta$ is associated to the stiffness in fiber direction. In a small strain framework, i.e. replacing $\log \boldsymbol{U}$ by $\varepsilon=\frac{1}{2}\left(\operatorname{Grad} \boldsymbol{u}+\operatorname{Grad}^{T} \boldsymbol{u}\right)$, the above given energy function would refer to transversely isotropic linear elasticity. In an exponential framework the exponentiated transversely isotropic strain-energy function

$$
\begin{aligned}
W_{2}(\log \boldsymbol{U})= & \frac{\mu_{\mathrm{T}}}{k_{1}} \exp \left[k_{1}\|\operatorname{dev} \log \boldsymbol{U}\|^{2}\right]+\frac{\kappa}{2 k_{2}} \exp \left[k_{2}(\operatorname{tr} \log \boldsymbol{U})^{2}\right] \\
& +\frac{\alpha}{k_{3}} \exp \left[k_{3}\langle\log \boldsymbol{U}, \boldsymbol{M}\rangle(\operatorname{tr} \log \boldsymbol{U})\right]+\frac{2\left(\mu_{\mathrm{L}}-\mu_{\mathrm{T}}\right)}{k_{4}} \exp \left[k_{4}\left\langle\boldsymbol{M},(\log \boldsymbol{U})^{2}\right\rangle\right] \\
& +\frac{\beta}{2 k_{5}} \exp \left[k_{5}\langle\boldsymbol{M}, \log \boldsymbol{U}\rangle^{2}\right]
\end{aligned}
$$


will result in the same elasticity tensor $\left.\mathbb{C}\right|_{\boldsymbol{C}=\mathbf{1}}$ at the identity, whereby $k_{i}>0$ are further non-dimensional parameters. We also note that while the isotropic invariants have the proposed differential geometric meaning, the novel exponential terms are formulated on an ad hoc basis.

Due to the non-linearity of the above given equations we aim to identify the general material parameters in the reference configuration with $\boldsymbol{C}=\mathbf{1}$ and $\log \boldsymbol{U}=\mathbf{0}$. In case of linearized transversely isotropic materials the elasticity tensor $\mathbb{C}$ may be formulated in terms of five material parameters, see Eq. (43). If we choose the isotropic-plane to be spanned by the $X_{1}$ and $X_{2}$ axis and the preferred direction to coincide with the coordinate axis $X_{3}$, perpendicular to the isotropic plane, and $\boldsymbol{M}=\operatorname{diag}(0,0,1)$ we may write

$$
\mathbb{C}^{\mathrm{V}}=\left(\begin{array}{cccccc}
\mathbb{C}_{11}^{\mathrm{V}} & \mathbb{C}_{12}^{\mathrm{V}} & \mathbb{C}_{13}^{\mathrm{V}} & 0 & 0 & 0 \\
\mathbb{C}_{12}^{\mathrm{V}} & \mathbb{C}_{11}^{\mathrm{V}} & \mathbb{C}_{13}^{\mathrm{V}} & 0 & 0 & 0 \\
\mathbb{C}_{13}^{\mathrm{V}} & \mathbb{C}_{13}^{\mathrm{V}} & \mathbb{C}_{33}^{\mathrm{V}} & 0 & 0 & 0 \\
0 & 0 & 0 & \frac{1}{2}\left(\mathbb{C}_{11}^{\mathrm{V}}-\mathbb{C}_{12}^{\mathrm{V}}\right) & 0 & 0 \\
0 & 0 & 0 & 0 & \mathbb{C}_{44}^{\mathrm{V}} & 0 \\
0 & 0 & 0 & 0 & 0 & \mathbb{C}_{44}^{\mathrm{V}}
\end{array}\right)
$$

in Voigt-notation. In the reference configuration, the formulated transversely isotropic strain energy functions $W_{1}$ and $W_{2}$, both yield

$$
\left.\mathbb{C}^{\mathrm{V}}\right|_{\boldsymbol{C}=\mathbf{1}}=\left(\begin{array}{cccccc}
\lambda+2 \mu_{\mathrm{T}} & \lambda & \lambda+\alpha & 0 & 0 & 0 \\
\lambda & \lambda+2 \mu_{\mathrm{T}} & \lambda+\alpha & 0 & 0 & 0 \\
\lambda+\alpha & \lambda+\alpha & \lambda-2 \mu_{\mathrm{T}}+2 \alpha+4 \mu_{\mathrm{L}}+\beta & 0 & 0 & 0 \\
0 & 0 & 0 & \mu_{\mathrm{T}} & 0 & \\
0 & 0 & 0 & 0 & \mu_{\mathrm{L}} & 0 \\
0 & 0 & 0 & 0 & 0 & \mu_{\mathrm{L}}
\end{array}\right)
$$

where the conversion $\lambda=\left(3 \kappa-2 \mu_{T}\right) / 3$ was used. The components of the above presented scheme are related to the five classical components in Eq. 43. through

$$
\begin{aligned}
\mu_{\mathrm{L}} & =\mathbb{C}_{44}^{\mathrm{V}} \\
\mu_{\mathrm{T}} & =\frac{1}{2}\left(\mathbb{C}_{11}^{\mathrm{V}}-\mathbb{C}_{12}^{\mathrm{V}}\right) \\
\lambda & =\mathbb{C}_{12}^{\mathrm{V}} \\
\alpha & =\mathbb{C}_{13}^{\mathrm{V}}-\mathbb{C}_{12}^{\mathrm{V}} \\
\beta & =\mathbb{C}_{11}^{\mathrm{V}}+\mathbb{C}_{33}^{\mathrm{V}}-2 \mathbb{C}_{13}^{\mathrm{V}}-4 \mathbb{C}_{44}^{\mathrm{V}}
\end{aligned}
$$

in analogy to Schröder and Gross [54]. The anisotropic characteristic of the strain energy function $W_{2}$ in Eq. (42) will be outlined on a number of numerical examples, where different material parameters according to Table 2 are used.

Further, the sets 4,5 and 6 are chosen such that only one term involving a structural tensor in Eq. (42) is active. Therefore, Set 4 is directly associated with the term $\langle\log \boldsymbol{U}, \boldsymbol{M}\rangle(\operatorname{tr} \log \boldsymbol{U})$, Set 5 with $\langle\log \boldsymbol{U}, \boldsymbol{M}\rangle$ and Set 6 with $\left\langle(\log \boldsymbol{U})^{2}, \boldsymbol{M}\right\rangle=$ $\|\operatorname{dev}(\log \boldsymbol{U}) \boldsymbol{M}\|^{2}$. In contrast, Set 3 will serve as the isotropic reference case. In order to obtain comparable results a similar level of distinct anisotropy is chosen for each set. 


\begin{tabular}{|c|c|c|c|c|c|c|c|c|c|c|}
\hline Set & $\lambda$ & $\mu_{\mathrm{T}}$ & $\alpha$ & $\beta$ & $\mu_{\mathrm{L}}$ & $k_{1}$ & $k_{2}$ & $k_{3}$ & $k_{4}$ & $k_{5}$ \\
\hline Set 1 & 1000 & 175 & 10 & 10 & 375 & 1 & 1 & 1 & 1 & 1 \\
Set 2 & 5.64 & 2.64 & 1.27 & 0.29 & 5.66 & 1 & 1 & 1 & 1 & 1 \\
Set 3 & 5.5 & 2.5 & 0.00 & 0.00 & 2.5 & 1 & 1 & 75 & 25 & 45 \\
Set 4 & 5.5 & 14 & 40.75 & 0.00 & 14 & 1 & 1 & 75 & 25 & 45 \\
Set 5 & 5.5 & 2.5 & 0.00 & 104.5 & 2.5 & 1 & 1 & 75 & 25 & 45 \\
Set 6 & 5.5 & 2.5 & 0.00 & 0.00 & 28.625 & 1 & 1 & 75 & 25 & 45 \\
\hline
\end{tabular}

Table 2: Different parameter sets for the numerical examples.

To achieve this, $\mathbb{C}_{33}^{\mathrm{V}}$ is the same for all three sets regarding the reference configuration. All parameter sets have been checked to be positive definite for $\left.\mathbb{C}^{\mathrm{V}}\right|_{\boldsymbol{C}=\mathbf{1}}$. Note that in case of Set 4 the parameter $\mu_{\mathrm{T}}$ needed to be increased in order to guarantee the positive definiteness of $\left.\mathbb{C}^{\mathrm{V}}\right|_{\boldsymbol{C}=\mathbf{1}}$. The corresponding matrices are listed below:

$$
\begin{aligned}
\text { Set } 4:\left.\mathbb{C}^{\mathrm{V}}\right|_{\boldsymbol{C = 1}} & =\left(\begin{array}{cccccc}
33.5 & 5.5 & 46.25 & 0 & 0 & 0 \\
5.5 & 33.5 & 46.25 & 0 & 0 & 0 \\
46.25 & 46.25 & 115 & 0 & 0 & 0 \\
0 & 0 & 0 & 14 & 0 & 0 \\
0 & 0 & 0 & 0 & 14 & 0 \\
0 & 0 & 0 & 0 & 0 & 14
\end{array}\right) \\
\text { Set 5: }\left.\mathbb{C}^{\mathrm{V}}\right|_{\boldsymbol{C}=\mathbf{1}} & =\left(\begin{array}{cccccc}
10.5 & 5.5 & 5.5 & 0 & 0 & 0 \\
5.5 & 10.5 & 5.5 & 0 & 0 & 0 \\
5.5 & 5.5 & 115 & 0 & 0 & 0 \\
0 & 0 & 0 & 2.5 & 0 & 0 \\
0 & 0 & 0 & 0 & 2.5 & 0 \\
0 & 0 & 0 & 0 & 0 & 2.5
\end{array}\right) \\
\text { Set } 6:\left.\mathbb{C}^{\mathrm{V}}\right|_{\boldsymbol{C}=\mathbf{1}} & =\left(\begin{array}{ccccccc}
10.5 & 5.5 & 5.5 & 0 & 0 & 0 \\
5.5 & 10.5 & 5.5 & 0 & 0 & 0 \\
5.5 & 5.5 & 115 & 0 & 0 & 0 \\
0 & 0 & 0 & 2.5 & 0 & 0 \\
0 & 0 & 0 & 0 & 28.625 & 0 \\
0 & 0 & 0 & 0 & 0 & 28.625
\end{array}\right)
\end{aligned}
$$

The implementation in a finite element framework in this work was done according to the formulation in Schröder et al. [56] and Löblein et al. [36]. The weak form of balance of momentum

$$
G(\boldsymbol{u}, \delta \boldsymbol{u}):=\int_{\mathcal{B}}\left\langle\operatorname{Div}\left(\partial_{\boldsymbol{F}} \psi(\boldsymbol{F})\right)+\rho_{0}(\boldsymbol{b}-\ddot{\boldsymbol{x}}), \delta \boldsymbol{u}\right\rangle \mathrm{d} V
$$

required for the finite element code may be reformulated such that we obtain

$$
G(\boldsymbol{u}, \delta \boldsymbol{u})=\underbrace{\int_{\mathcal{B}}\langle\boldsymbol{\tau}, \nabla(\delta \boldsymbol{u})\rangle \mathrm{d} V}_{G^{\text {int }}}-\underbrace{\left(\int_{\partial \mathcal{B}_{t}}\langle\mathbf{f}, \delta \boldsymbol{u}\rangle \mathrm{d} A+\int_{\mathcal{B}}\left\langle\rho_{0}(\boldsymbol{b}-\ddot{\boldsymbol{x}}), \delta \boldsymbol{u}\right\rangle \mathrm{d} V\right)}_{G^{\text {ext }}}=0 .
$$

Here, the body force in the reference configuration is denoted by $\mathbf{f}, \delta \boldsymbol{u}$ is the variation of the displacement field and $\ddot{\boldsymbol{x}}$ the acceleration. For the solution scheme in a finite element 
framework a Newton iteration is required. Therefore, the linearization

$$
\operatorname{Lin} G(\overline{\boldsymbol{u}}, \delta \boldsymbol{u}, \Delta \boldsymbol{u}):=G(\overline{\boldsymbol{u}}, \delta \boldsymbol{u})+\Delta G(\overline{\boldsymbol{u}}, \delta \boldsymbol{u}, \Delta \boldsymbol{u})
$$

at $\boldsymbol{u}=\overline{\boldsymbol{u}}$ is required where the increment $\Delta G$ is defined through

$$
\Delta G=\int_{\mathcal{B}}\left\langle\nabla_{\text {sym }}(\delta \boldsymbol{u}), \mathbb{C}: \nabla_{\text {sym }}(\Delta \boldsymbol{u})\right\rangle \mathrm{d} V+\int_{\mathcal{B}}\langle\nabla(\Delta \boldsymbol{u}) \boldsymbol{\tau}, \nabla(\delta \boldsymbol{u})\rangle \mathrm{dV},
$$

where the Eulerian tangent moduli $\mathbb{C}$ is obtained by the push-forward of the Lagrangian tangent moduli $\mathbb{C}$, i.e.

$$
\mathbb{C}=(\boldsymbol{F} \otimes \boldsymbol{F}): \mathbb{C}:\left(\boldsymbol{F}^{T} \otimes \boldsymbol{F}^{T}\right)
$$

and $\nabla_{\text {sym }}(\bullet)=1 / 2\left[\operatorname{grad}(\bullet)+\operatorname{grad}^{T}(\bullet)\right]$. For the numerical treatment the weak formulations of the aforementioned balance equation has been implemented in the finite element analysis program FEAP of R.L. Taylor, University of California. For all of the following examples quadratic triangular elements with six nodes per element were used.

Tension test: In a first example the transversely isotropic material behavior is to be explained on the basis of a tensile test under plane strain conditions, see Fig. 1 1a). Material parameter Set 1 was chosen and the preferred direction $\boldsymbol{A}$, defined with help of the fiber angle $\beta_{\mathrm{f}}$, was varied. The computed displacements of the nodes 1, 2 and 3 over the fiber angle are plotted in Fig. 1 $1 \mathrm{~b}$ ). The displacements $\delta_{\mathrm{h}_{1}}, \delta_{\mathrm{h}_{2}}$ and $\delta_{\mathrm{v}_{3}}$ are symmetric concerning $\beta_{\mathrm{f}}=90^{\circ}$, while the vertical displacements $\delta_{\mathrm{v}_{1}}$ and $\delta_{\mathrm{v}_{2}}$ are antisymmetric. Surprisingly, the horizontal displacements don't reach their maximum value for $\beta_{\mathrm{f}}=90^{\circ}$. The load $p_{0}$ has been chosen such that large deformations are present.

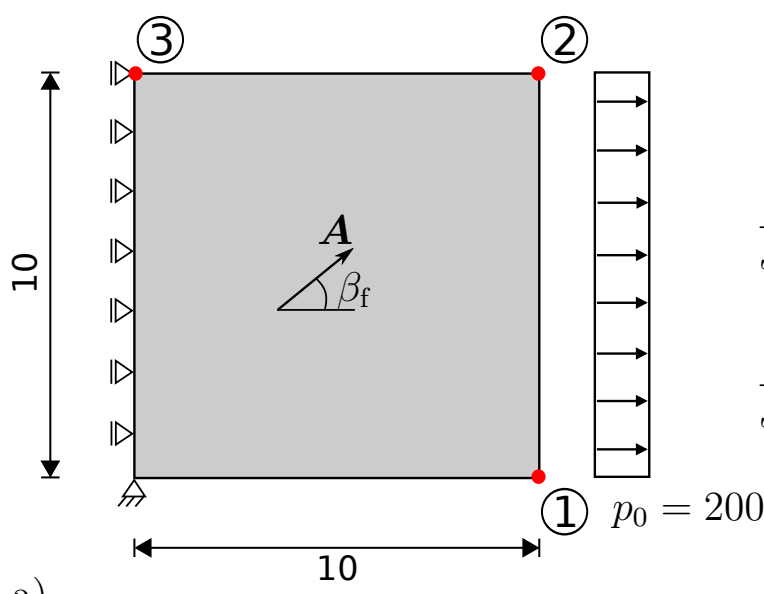

a)

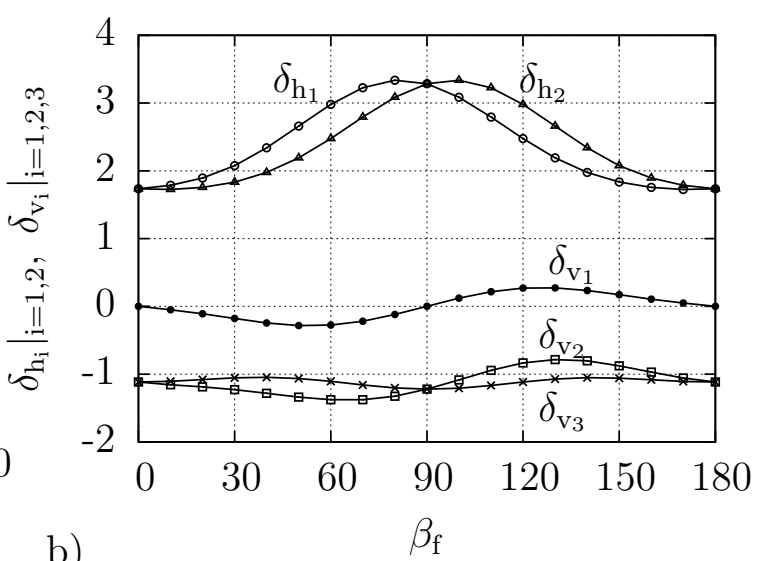

Figure 1: Example 1: a) Tension test with one preferred direction and varying fiber orientation. b) Nodal displacements depending on the fiber orientations are plotted for parameter Set 1.

Cooks Membrane: In a second example we consider the Cooks Membrane problem, as depicted in Fig. 2a) which is dominated by non-homogenous stress distributions. Again the fiber direction is to be varied and the body will undergo large deformations during loading. In Fig. 2 $\mathrm{b}$ ), the vertical displacements $\delta_{\mathrm{V}}$ of the node at the top right are plotted for different fiber angles. Parameter Set 2 was considered. The anisotropic effect due to the different fiber orientation clearly becomes apparent. 


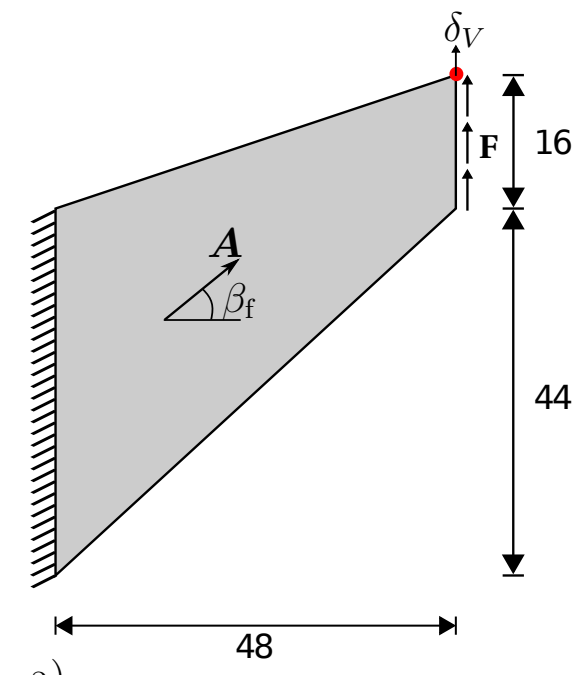

a)

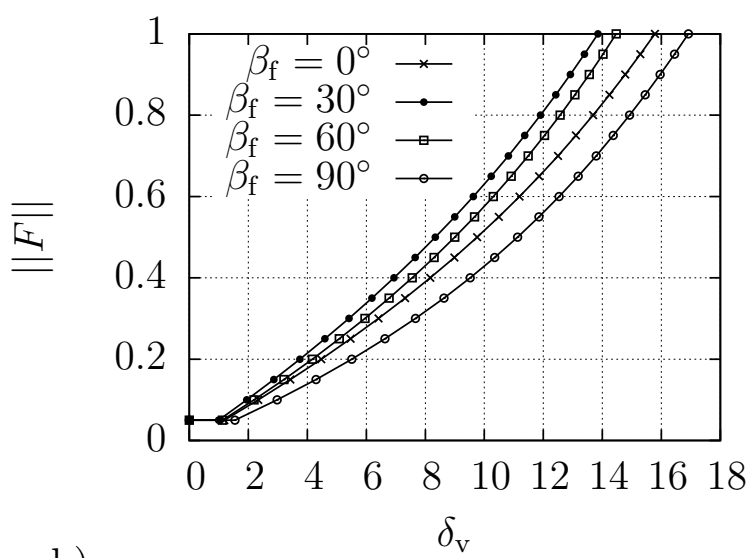

b)

Figure 2: Example 2: a) Cooks Membrane with one preferred direction and varying fiber orientation. b) Plot of the vertical displacement for different fiber orientations and parameter Set 2 .

Perforated Plate: Lastly, we want to consider three perforated plates, again under consideration of plane strain conditions. The first two plates are squares and share the same geometry. The first one, referred to as Example 3a, is subject to uniaxial tensile loads, see Fig. 3a), while the second one, referred to as example 3b, is subject to biaxial tensile loads, see Fig. 4a). In example 3c we consider a circular disk, depicted in Fig. 5a), which is expanded on the inner ring, i.e. we apply a radial displacement of 3.5. All simulations are displacement driven, i.e. only boundary conditions of Dirichlet type are present. Further, all three bodies have one preferred direction $\boldsymbol{A}$ with an angle of $45^{\circ}$ to the horizontal axis. The deformed bodies for the parameter Sets 3, 4, 5 and 6 are plotted next to the boundary value problems in Fig. 3, Fig. 4 and Fig. 5. The contour plots of the squares show the horizontal displacements $u_{1}$. In order to highlight the anisotropic characteristic of the circular disk, the circumferential stretch $\lambda_{\phi}=\sqrt{\left\langle\boldsymbol{C}, \boldsymbol{N}_{\varphi} \otimes \boldsymbol{N}_{\varphi}\right\rangle}$, where $\boldsymbol{N}_{\varphi}$ denotes the circumferential direction, is plotted for the third plate. The black lines in each plot indicate the shape of the body in the undeformed configuration.

The comparison of the different parameter sets are intended to demonstrate different anisotropic characteristics of different strain measures. Therefore, the parameter sets were chosen such that only one anisotropic part of the strain energy in Eq. (42) is active. First of all from Fig. 3 and Fig. 4 it becomes apparent that for the anisotropic Sets 4, 5 and 6 the displacements $u_{1}$ are not symmetric with respect to the horizontal axis, which is different for the isotropic Set 3. The different shapes of the holes very well emphasize that the usage of different anisotropic invariants may lead to very different deformations, even if the preferred direction is identical. In example 3c the inner ring of the disk is exposed to predefined deformations, which is why the shapes of the holes are the same for each parameter set. Nevertheless, the distribution of the circumferential stretch $\lambda_{\varphi}$ is considerably different. Considering a polar coordinate system the circumferential stretch is only depending on the radius, but independent of the polar angle for the isotropic Set 3. For Set 4 and Set 6 it can be seen that $\lambda_{\varphi}$ is smallest in the regions were the preferred direction $\boldsymbol{A}$ and the circumferential direction $\boldsymbol{N}_{\varphi}$ coincide and largest in the regions were $\boldsymbol{A}$ and $\boldsymbol{N}_{\varphi}$ are perpendicular. For Set 5 only the first of these two observations holds true. 
We conclude that a considerable stiffening effect in the preferred direction is visible for Set 4,5 and 6 .
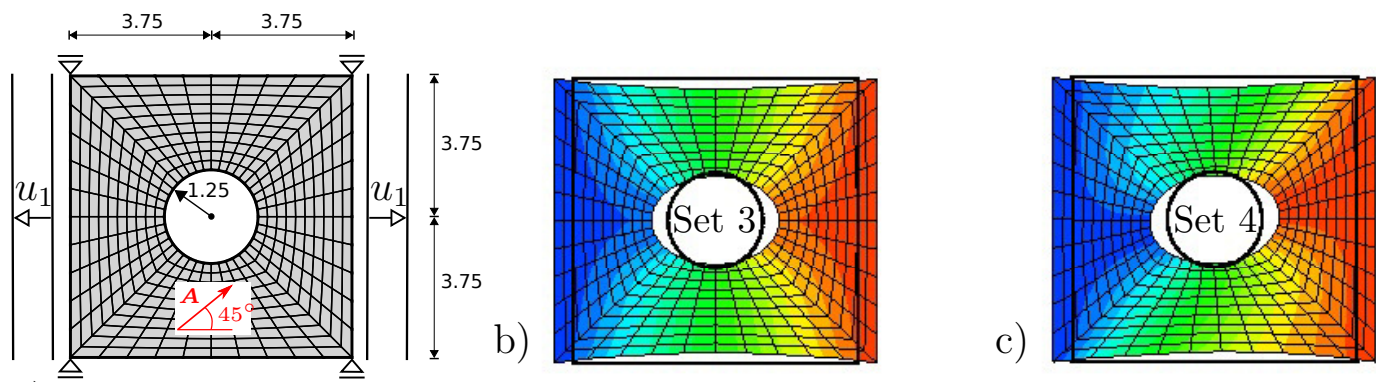

a)

d)

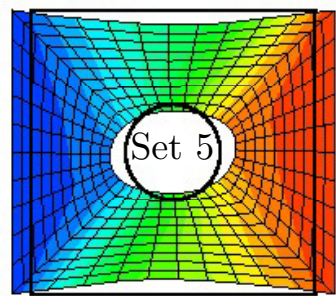

e)

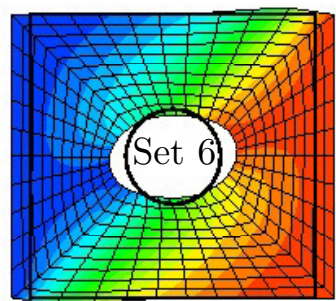

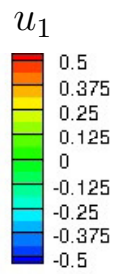

Figure 3: Example 3a: a) Boundary conditions and b)-e) deformed bodies of a perforated plate under uniaxial tension for parameter Set $3,4,5$ and 6 .
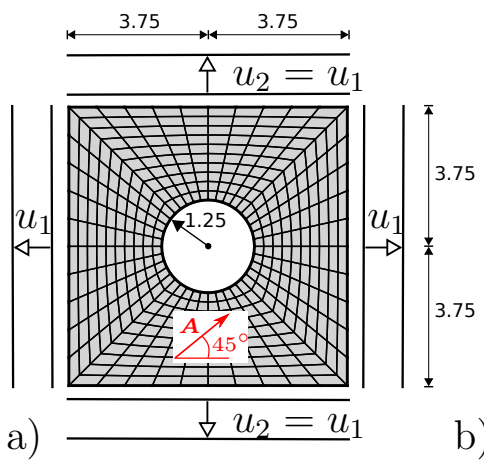

d)
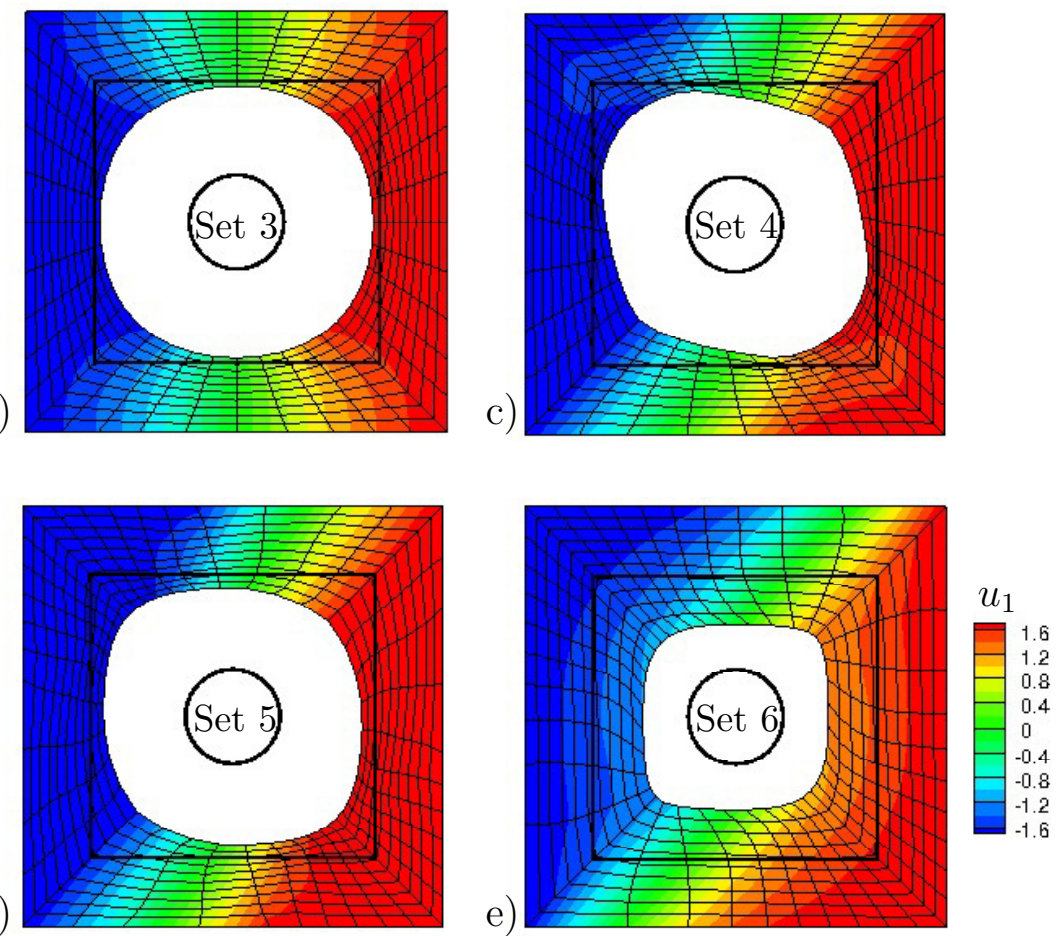

Figure 4: Example 3b: a) Boundary conditions and b)-e) deformed bodies of a perforated plate under biaxial tension for parameter Set $3,4,5$ and 6 . 


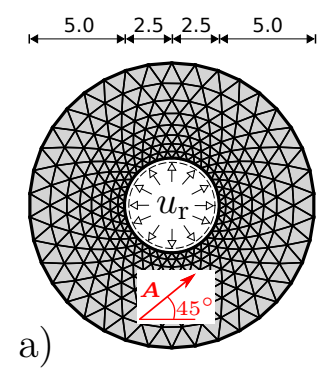

b)

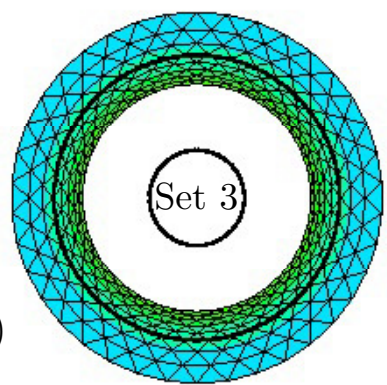

d)

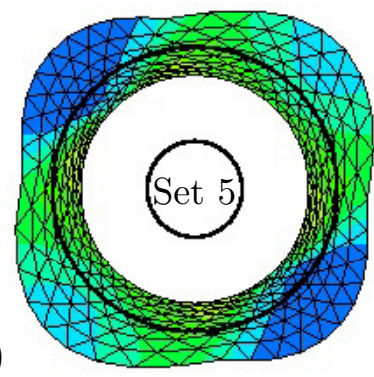

c)

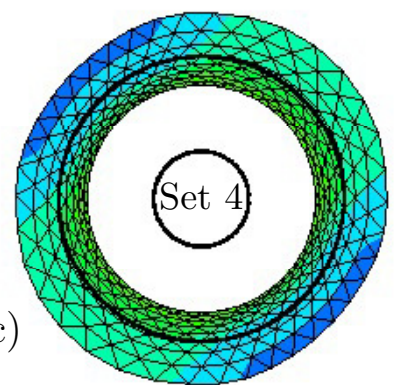

e)

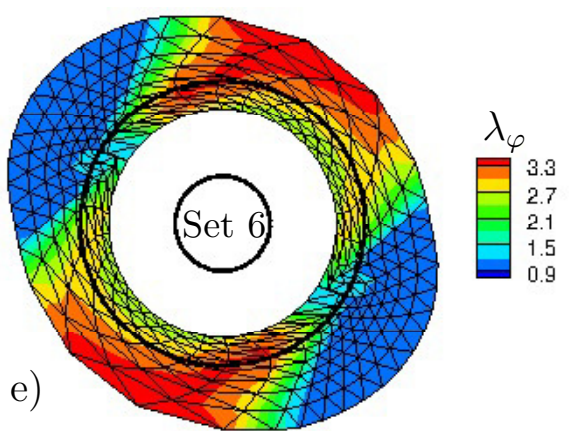

Figure 5: Example 3c: a) Boundary conditions and b)-e) deformed bodies of a circular perforated plate for parameter Set $3,4,5$ and 6 .

\subsection{Orthotropic Hencky and exponentiated Hencky models}

Orthotropic material behavior is symmetric regarding three orthogonal planes. These three planes are spanned by the three preferred directions $\boldsymbol{A}_{1}, \boldsymbol{A}_{2}$ and $\boldsymbol{A}_{3}$, which are unit vectors. The material symmetry group is then defined by

$$
\mathcal{G}_{o}:=\left\{ \pm \mathbf{1} ; \boldsymbol{R}_{1}, \boldsymbol{R}_{2}, \boldsymbol{R}_{3}\right\}
$$

where $\boldsymbol{R}_{1}, \boldsymbol{R}_{2}$ and $\boldsymbol{R}_{3}$ are the reflections with respect to the planes spanned by $\left(\boldsymbol{A}_{2}, \boldsymbol{A}_{3}\right)$, $\left(\boldsymbol{A}_{1}, \boldsymbol{A}_{3}\right)$ and $\left(\boldsymbol{A}_{1}, \boldsymbol{A}_{2}\right)$, respectively. The three preferred directions $\boldsymbol{A}_{i} \mid i=1,2,3$ are orthogonal and form the three structural tensors $\boldsymbol{M}_{i}=\boldsymbol{A}_{i} \otimes \boldsymbol{A}_{i} \mid i=1,2,3$, which satisfy the orthogonality condition $\left\langle\boldsymbol{M}_{i}, \boldsymbol{M}_{j}\right\rangle=\delta_{i j}$. However, it is sufficient to formulate orthotropic strain energies with two structural tensors $\boldsymbol{M}_{1}$ and $\boldsymbol{M}_{2}$ and additional isotropic principal or main invariants. In the same manner as in the previous section we may formulate the orthotropic energy function

$$
\begin{aligned}
W_{3}(\log \boldsymbol{U})= & \mu\|\operatorname{dev} \log \boldsymbol{U}\|^{2}+\frac{\kappa}{2}[\operatorname{tr}(\log \boldsymbol{U})]^{2} \\
& +\alpha_{1}\left\langle\log \boldsymbol{U}, \boldsymbol{M}_{1}\right\rangle(\operatorname{tr} \log \boldsymbol{U})+\alpha_{2}\left\langle\log \boldsymbol{U}, \boldsymbol{M}_{2}\right\rangle(\operatorname{tr} \log \boldsymbol{U}) \\
& +2 \mu_{1}\left\langle\boldsymbol{M}_{1},(\log \boldsymbol{U})^{2}\right\rangle+2 \mu_{2}\left\langle\boldsymbol{M}_{2},(\log \boldsymbol{U})^{2}\right\rangle \\
& +\frac{1}{2} \beta_{1}\left\langle\boldsymbol{M}_{1}, \log \boldsymbol{U}\right\rangle^{2}+\frac{1}{2} \beta_{2}\left\langle\boldsymbol{M}_{2}, \log \boldsymbol{U}\right\rangle^{2}+\frac{1}{2} \beta_{3}\left\langle\boldsymbol{M}_{1}, \log \boldsymbol{U}\right\rangle\left\langle\boldsymbol{M}_{2}, \log \boldsymbol{U}\right\rangle
\end{aligned}
$$


and the corresponding exponentiated version

$$
\begin{aligned}
W_{4}(\log \boldsymbol{U})= & \frac{\mu}{k_{1}} \exp \left[k_{1}\|\operatorname{dev} \log \boldsymbol{U}\|^{2}\right]+\frac{\kappa}{2 k_{2}} \exp \left[k_{2}(\operatorname{tr} \log \boldsymbol{U})^{2}\right] \\
& +\frac{\alpha_{1}}{k_{3}} \exp \left[k_{3}\left\langle\log \boldsymbol{U}, \boldsymbol{M}_{1}\right\rangle(\operatorname{tr} \log \boldsymbol{U})\right]+\frac{\alpha_{1}}{k_{4}} \exp \left[k_{4}\left\langle\log \boldsymbol{U}, \boldsymbol{M}_{2}\right\rangle(\operatorname{tr} \log \boldsymbol{U})\right] \\
& +\frac{2 \mu_{1}}{k_{5}} \exp \left[k_{5}\left\langle\boldsymbol{M}_{1},(\log \boldsymbol{U})^{2}\right\rangle\right]+\frac{2 \mu_{2}}{k_{6}} \exp \left[k_{6}\left\langle\boldsymbol{M}_{2},(\log \boldsymbol{U})^{2}\right\rangle\right] \\
& +\frac{\beta_{1}}{2 k_{7}} \exp \left[k_{7}\left\langle\boldsymbol{M}_{1}, \log \boldsymbol{U}\right\rangle^{2}\right]+\frac{\beta_{2}}{2 k_{8}} \exp \left[k_{8}\left\langle\boldsymbol{M}_{2}, \log \boldsymbol{U}\right\rangle^{2}\right] \\
& +\frac{\beta_{3}}{2 k_{9}} \exp \left[k_{9}\left\langle\boldsymbol{M}_{1}, \log \boldsymbol{U}\right\rangle\left\langle\boldsymbol{M}_{2}, \log \boldsymbol{U}\right\rangle\right]
\end{aligned}
$$

The orthotropic elasticity tensor $\mathbb{C}^{\mathrm{V}}$ has nine independent variables. Choosing the structural tensors $\boldsymbol{M}_{1}=\operatorname{diag}(1,0,0), \boldsymbol{M}_{2}=\operatorname{diag}(0,1,0)$ we obtain the general form

$$
\mathbb{C}^{\mathrm{V}}=\left(\begin{array}{cccccc}
\mathbb{C}_{11}^{\mathrm{V}} & \mathbb{C}_{12}^{\mathrm{V}} & \mathbb{C}_{13}^{\mathrm{V}} & 0 & 0 & 0 \\
\mathbb{C}_{12}^{\mathrm{V}} & \mathbb{C}_{22}^{\mathrm{V}} & \mathbb{C}_{23}^{\mathrm{V}} & 0 & 0 & 0 \\
\mathbb{C}_{13}^{\mathrm{V}} & \mathbb{C}_{23}^{\mathrm{V}} & \mathbb{C}_{33}^{\mathrm{V}} & 0 & 0 & 0 \\
0 & 0 & 0 & \mathbb{C}_{44}^{\mathrm{V}} & 0 & 0 \\
0 & 0 & 0 & 0 & \mathbb{C}_{55}^{\mathrm{V}} & 0 \\
0 & 0 & 0 & 0 & 0 & \mathbb{C}_{66}^{\mathrm{V}}
\end{array}\right)
$$

The material tangent of both functions, $W_{3}$ and $W_{4}$ both yield

$$
\left.\mathbb{C}^{\mathrm{V}}\right|_{\boldsymbol{C}=\mathbf{1}}=\left(\begin{array}{cccccc}
2 \mu+\lambda+ & \lambda+\alpha_{1}+\alpha_{2}+\beta_{3} & \lambda+\alpha_{1} & 0 & 0 & 0 \\
2 \alpha_{1}+4 \mu_{1}+\beta_{1} & 2 \mu+\lambda+ & & & & \\
\lambda+\alpha_{1}+\alpha_{2}+\beta_{3} & 2 \alpha_{2}+4 \mu_{2}+\beta_{2} & \lambda+\alpha_{2} & 0 & 0 & 0 \\
\lambda+\alpha_{1} & \lambda+\alpha_{2} & 2 \mu+\lambda & 0 & 0 & 0 \\
0 & 0 & 0 & \mu+\mu_{2} & 0 & 0 \\
0 & 0 & 0 & 0 & \mu+\mu_{1} & 0 \\
0 & 0 & 0 & 0 & 0 & \mu+\mu_{1}+\mu_{2}
\end{array}\right)
$$

in the reference configuration, with $\lambda=(3 \kappa-2 \mu) / 3$. Following the same scheme as in the transversely isotropic case the parameter identification gives the following relations

$$
\begin{aligned}
\mu & =\mathbb{C}_{44}^{\mathrm{V}}+\mathbb{C}_{55}^{\mathrm{V}}-\mathbb{C}_{66}^{\mathrm{V}} \\
\mu_{1} & =\mathbb{C}_{66}^{\mathrm{V}}-\mathbb{C}_{44}^{\mathrm{V}} \\
\mu_{2} & =\mathbb{C}_{66}^{\mathrm{V}}-\mathbb{C}_{55}^{\mathrm{V}} \\
\lambda & =\mathbb{C}_{33}^{\mathrm{V}}+2\left(\mathbb{C}_{66}^{\mathrm{V}}-\mathbb{C}_{44}^{\mathrm{V}}-\mathbb{C}_{55}^{\mathrm{V}}\right) \\
\alpha_{1} & =\mathbb{C}_{13}^{\mathrm{V}}-\mathbb{C}_{33}^{\mathrm{V}}-2\left(\mathbb{C}_{66}^{\mathrm{V}}-\mathbb{C}_{44}^{\mathrm{V}}-\mathbb{C}_{55}^{\mathrm{V}}\right) \\
\alpha_{2} & =\mathbb{C}_{23}^{\mathrm{V}}-\mathbb{C}_{33}^{\mathrm{V}}-2\left(\mathbb{C}_{66}^{\mathrm{V}}-\mathbb{C}_{44}^{\mathrm{V}}-\mathbb{C}_{55}^{\mathrm{V}}\right) \\
\beta_{1} & =\mathbb{C}_{11}^{\mathrm{V}}+\mathbb{C}_{33}^{\mathrm{V}}-2 \mathbb{C}_{13}^{\mathrm{V}}-4 \mathbb{C}_{55}^{\mathrm{V}} \\
\beta_{2} & =\mathbb{C}_{22}^{\mathrm{V}}+\mathbb{C}_{33}^{\mathrm{V}}-2 \mathbb{C}_{23}^{\mathrm{V}}-4 \mathbb{C}_{44}^{\mathrm{V}} \\
\beta_{3} & =\mathbb{C}_{12}^{\mathrm{V}}-\mathbb{C}_{13}^{\mathrm{V}}-\mathbb{C}_{23}^{\mathrm{V}}+\mathbb{C}_{33}^{\mathrm{V}}+2\left(\mathbb{C}_{66}^{\mathrm{V}}-\mathbb{C}_{44}^{\mathrm{V}}-\mathbb{C}_{55}^{\mathrm{V}}\right)
\end{aligned}
$$

A viscoelastic, orthotropic material model based on finite logarithmic strains has been recently proposed by Latorre and Montáns [35]. 


\subsection{Case study of the transversely isotropic model in logarithmic strain space}

In order to study the anisotropic properties under compression we further introduce the strain energy functions

$$
\begin{aligned}
& \psi_{\mathbf{\Xi}_{\mathrm{C}}}^{\mathrm{ti}}=\frac{\mu_{1}}{2 k_{1}}\left\{\exp \left[k_{1}(\underbrace{\left\langle\boldsymbol{C}^{i}, \boldsymbol{M}\right\rangle}_{I_{4}^{\mathrm{C}^{i}}}-1)^{2}\right]-1\right\}, \\
& \psi_{\mathbf{\Theta}_{\mathrm{H}}^{\mathrm{ti}}}^{\mathrm{ti}}=\frac{\mu_{1}}{2 k_{1}}\left\{\exp [k_{1} \underbrace{\left\langle(\log \boldsymbol{U})^{i}, \boldsymbol{M}\right\rangle}_{I_{4}^{\mathrm{H}^{i}}}{ }^{2}]-1\right\} .
\end{aligned}
$$

In the following we aim to investigate the performance of the anisotropic invariants $I_{4}^{\mathrm{C}^{i}}$, $I_{4}^{\mathrm{H}^{i}}$, see Eq. (26), respectively. The evolution of the invariants, as well as the stress response of the transversely isotropic strain energy functions $\psi^{\mathrm{ti}}\left(I_{4}^{\mathrm{C}^{i}}\right)$ and $\psi^{\mathrm{ti}}\left(I_{4}^{\mathrm{H}^{i}}\right)$, are plotted for different loading scenarios. The examples are evaluated such that the results are independent of any chosen isotropic strain energy function, since only the anisotropic stress response will be plotted. The case distinction for compression and tension included in the energy functions will be neglected, i.e. the fibers are allowed to induce stresses under compression and the energy functions according to Eq. (55) and Eq. (56) will be used. The parameters $\mu_{1}$ and $k_{1}$ are set to one and the plotted evolution of the Cauchy stress

$$
\boldsymbol{\sigma}^{\text {aniso }}=\frac{1}{J} 2 \boldsymbol{F} \frac{\partial \psi^{\text {ti }}}{\partial \boldsymbol{C}} \boldsymbol{F}^{T}
$$

will be normalized by the occurring maximum stress at the final deformation state, to allow for a better comparison.

This study is restricted to classical homogenous deformation states, i.e. uniaxial tension and compression, simple shear and biaxial loading conditions. During uniaxial tension, uniaxial compression and biaxial loading the angles $\theta_{k}$ will remain constant and the body is free of rotations, i.e. $\boldsymbol{F}=\boldsymbol{U}$. Only during the shear test they will change with a change in the deformation.

4.3.1 Uniaxial tension and compression The considered problem is depicted in Fig. 6. In this case the fiber direction is aligned with the loading direction. The reference configuration as well as the deformed configurations under tension and compression are shown on the right. The component $F_{11}$ of the deformation gradient refers to the stretch in fiber direction. Since the body is considered to be incompressible we find that $F_{22}=$ $F_{33}=1 / \sqrt{F_{11}}$.

The results for the transversely anisotropic Hencky function $\psi_{\boldsymbol{\Theta}_{\mathrm{H}}}^{\mathrm{ti}}$ are displayed in Fig. 7 . As already discussed in the previous section, in Fig. 7a) it becomes apparent that for even exponents of $i$ in $I_{4}^{\mathrm{H}^{\mathrm{i}}}$ the values of the invariant are also positive under compression, i.e. the sign of the invariant is not the right choice to distinguish between tensile and compressive stretches. Nevertheless, the stress response seems to be adequate from a physical point of view for each of the considered invariants. For $I_{4}^{\mathrm{H}^{1}}=\langle\log \boldsymbol{U}, \boldsymbol{M}\rangle$ we obtain a perfectly linear material behavior. Due to the logarithmic framework the stress 
function is generally more sensitive to compression than to tension, see Fig. $7 \mathrm{~b}$ ). Note that the stress function was normalized with the corresponding highest stress $\max \left(\| \sigma_{11}^{\text {aniso }}\right) \|$ to allow for a better comparison. All stress-strain responses show the potential to exhibit significant strain stiffening for both tension and compression. This is especially remarkable for the compressive case. Hyun and Nakajima [31] for example found that porous copper fabricated by unidirectional solidification behaves strongly anisotropic under compression and exhibits considerable stiffening under large strains up to $80 \%$ due to the alignment of the pores. Classical anisotropic material laws based on the invariant $\left\langle\boldsymbol{C}^{i}, \boldsymbol{M}\right\rangle$ struggle to reproduce this effect. The normalized stresses under compression according to Eq. (55) are plotted in Fig. 8a). The plot reveals a strain softening behavior. Moreover, after a certain point the stresses will begin to increase although the body is further compressed which is strictly unphysical. Also classical polynomial laws of the form

$$
\psi_{\mathbf{3}_{\mathrm{C}}}^{\mathrm{ti}}=\frac{\mu_{1}}{2 k_{1}}\left(\left\langle\boldsymbol{C}^{i}, \boldsymbol{M}\right\rangle-1\right)^{k_{1}}
$$

suffer from this effect, see Fig. 8p). Here, $\mu_{1}$ was set to one and $k_{1}$ to two. In addition the domain of definition of the above energy is restricted to even values of $k_{1}$ in compression which significantly limits the parameter fitting properties.
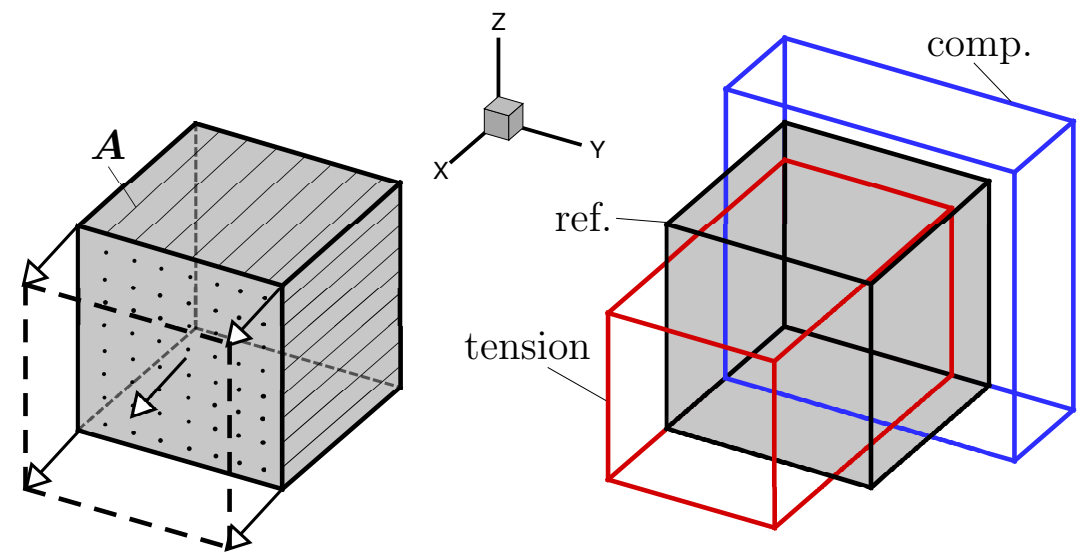

Figure 6: Uniaxial compression and tension test, where the preferred direction and the loading are aligned. The body is assumed to be incompressible with $V_{\text {ref }}=V_{\text {tension }}=V_{\text {comp }}$. 

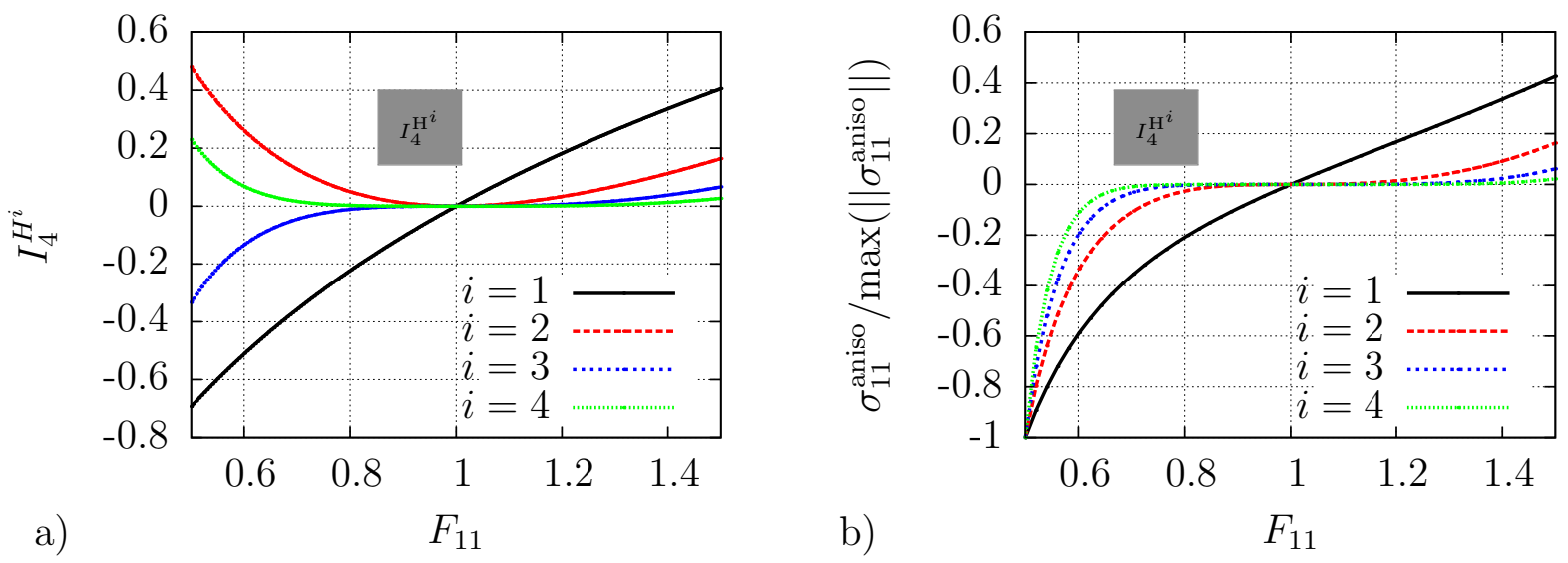

Figure 7: Monotonicity of the Cauchy stress $\sigma_{11}$ as function of uniaxial stretch. Evolution of a) the anisotropic Hencky-type invariants $I_{4}^{\mathrm{H}^{i}}$ for $i=1,2,3,4$ and b) corresponding anisotropic stress response under uniaxial tension and compression for $\psi_{\mathbf{\bigotimes}_{\mathrm{H}}^{\mathrm{ti}}}$. The stresses are normalized by the absolute value of the maximum arising compressive stress at $F_{11}=0.5$.
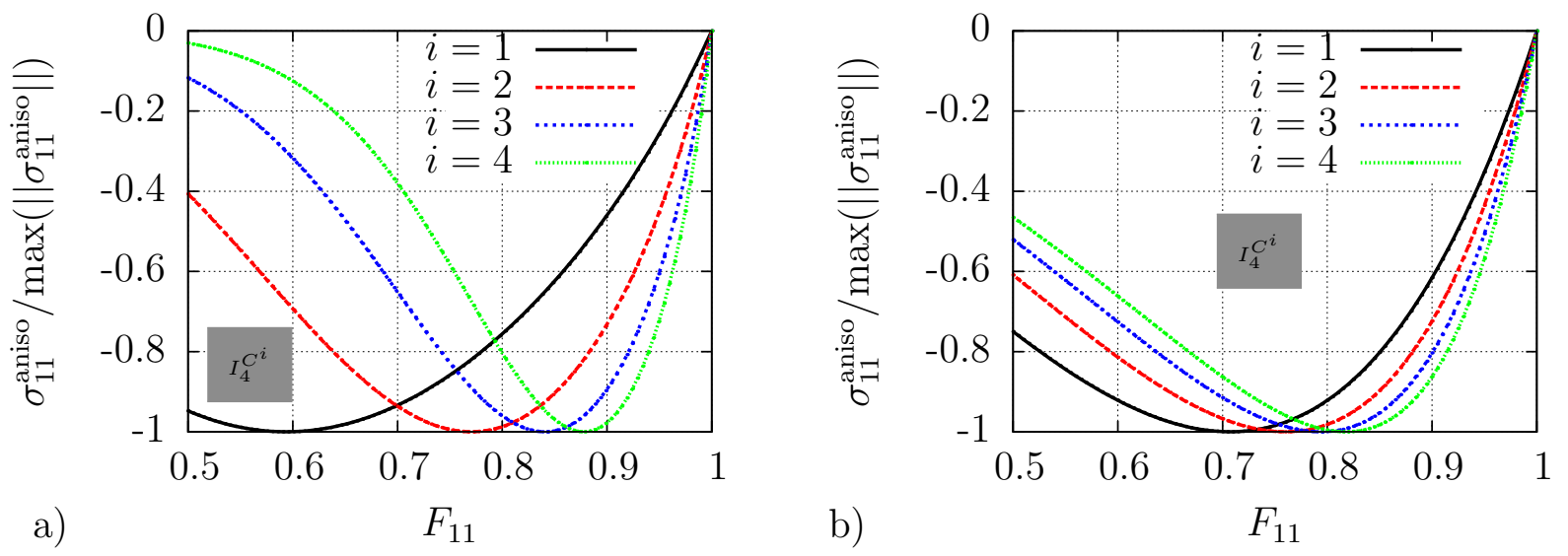

Figure 8: Pathological non-monotonicity of the Cauchy stress $\sigma_{11}$ as function of uniaxial stretch. Normalized anisotropic stress response under uniaxial compression. In a) the exponential strain energy function $\psi_{\mathbf{1}_{\mathrm{C}}^{\mathrm{ti}}}^{\mathrm{ti}}$ and in b) the polynomial function $\psi_{\mathbf{3}_{\mathrm{C}}^{\mathrm{ti}}}^{\mathrm{ts}}$ is plotted. 
4.3.2 Simple shear Next we investigate the behavior for simple shear, according to Fig. 9. The shear direction will be aligned with the fiber direction and the amount of shear

$$
\gamma=\frac{u}{L}
$$

is defined as the quotient of the displacements by the length. Note that in this example the fibers are not elongated at all, i.e. $I_{4}^{\mathrm{C}^{1}}=1$. The results for the transversely anisotropic Hencky function are displayed in Fig. 10 and Fig. 11. Again the invariants of even and odd powers take a different sign. The stress quantities which are not plotted in Fig. 11a) are equal to zero. That means for even powers $i, \sigma_{11}^{\text {aniso }}$ will be equal to zero.
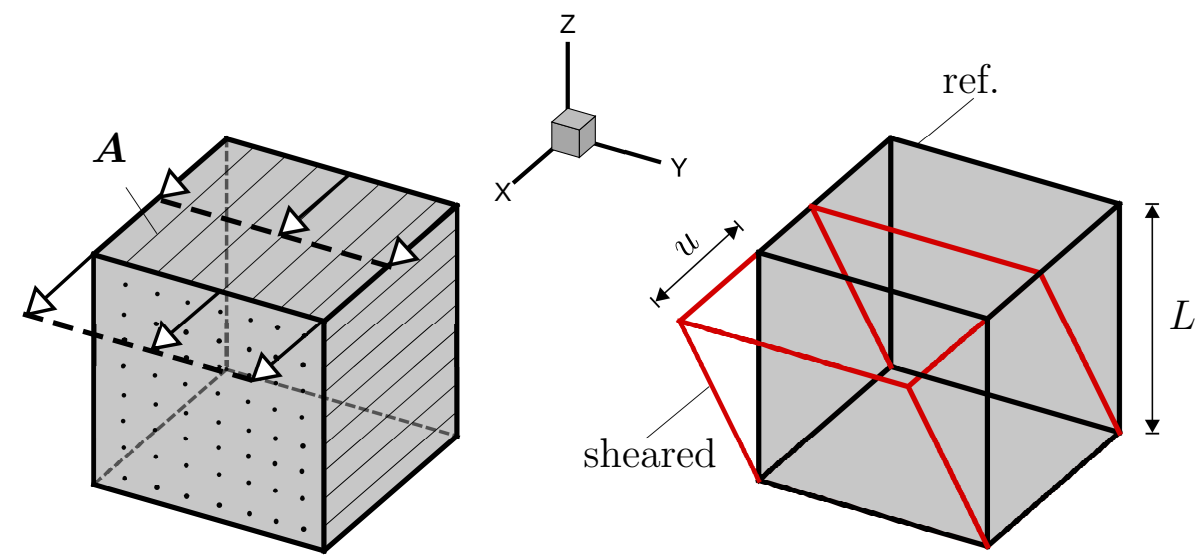

Figure 9: Shear test, where the preferred direction and the shear direction are aligned. Simple shear deformation is incompressible with $V_{\text {ref }}=V_{\text {sheared }}$.
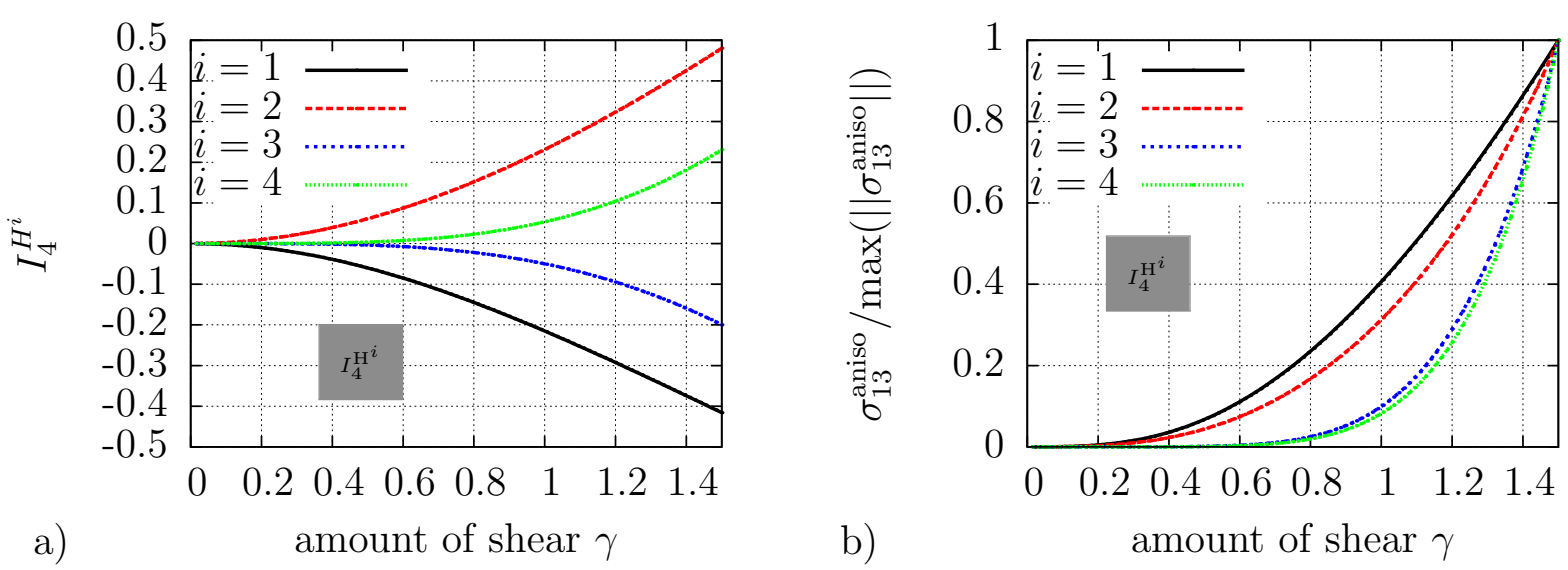

Figure 10: Evolution of the anisotropic Hencky-type invariants $I_{4}^{\mathrm{H}^{i}}$ for $i=1,2,3,4$ and b) corresponding Cauchy stresses $\sigma_{13}$ under simple shear. The stresses are normalized by the absolute value of the maximum arising shear stress at $\gamma=1.5$. 

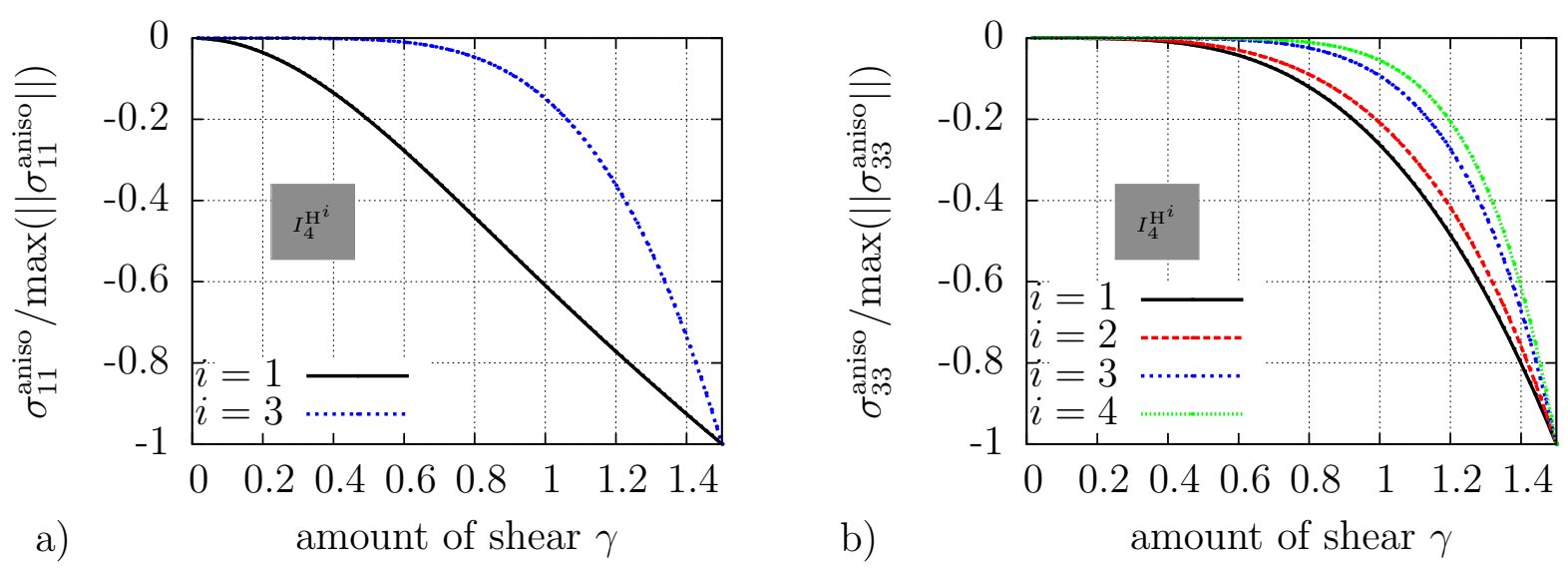

Figure 11: Evolution of the Cauchy stresses a) $\sigma_{11}$ and b) $\sigma_{33}$ corresponding to the anisotropic strain invariants plotted in Fig. 10p), under simple shear. Note that for $i=2$ and $i=4, \sigma_{11}$ is equal to zero.

4.3.3 Biaxial tension In this case the body is exposed to biaxial tensile displacements, see Fig. 12. We consider two fiber families which are orientated symmetric regarding the X-axis. Further, as indicated in the figure three different stretch ratios will be compared. The stretch ratio is here defined as the quotient $F_{11} / F_{22}$. For the three different stretch ratios the evolution of the quotient $\sigma_{22}^{\text {aniso }} / \sigma_{11}^{\text {aniso }}$ is plotted in Fig. 13,14 and 15 . In each of the figures we find the results for the anisotropic invariants of $\boldsymbol{C}^{i}$ on the left hand side and the results for $(\log \boldsymbol{U})^{i}$ on the right hand side. For the equi-biaxial test in Fig. 13 the stress ratio is the same for each model, at each time. The stress ratio directly follows from the fiber angle with respect to the x-axis to be $\sigma_{22}^{\text {aniso }} / \sigma_{11}^{\text {aniso }}=\tan ^{2} 30^{\circ}=1 / 3$. This ratio remains exactly the same for the other stretch ratios only if $I_{4}^{\mathrm{H}^{1}}$ is used, which seems to be unreasonable. Regarding the invariants of $\boldsymbol{C}$ we see that the starting point at nearly zero deformation is always defined by $\sigma_{22}^{\text {aniso }} / \sigma_{11}^{\text {aniso }}=1 / 3$, which is different for the Hencky-type strain measures. Further, the change in the stress ratio with increasing deformation is less pronounced in the case that Hencky strains are used. This behavior can be explained, when taking a look on Eq. (64). Since the angles $\theta_{l} \mid l=1,2,3$ are constant only the logarithmic stretches are of interest. Due to the logarithmic function the slope is decreasing when the stretch is increasing, i.e. for higher strains the slope is smaller than for lower strains which is also reflected by the shown stress ratios. Generally the slope of the stress ratios seem to have the opposite sign, regarding the basic strain measure. But all stresses appear to have the same sign, independent of the stress measure.

The stress ratios of the $I_{4}^{\mathrm{C}^{1}}$ model can be exactly reproduced by the computation of the fiber angle

$$
\beta_{\text {act }}=\arccos \left(\frac{\left\langle\boldsymbol{F} \boldsymbol{A}, \boldsymbol{e}_{\mathrm{x}}\right\rangle}{\|\boldsymbol{F} \boldsymbol{A}\|\left\|\boldsymbol{e}_{\mathrm{x}}\right\|}\right),
$$

where $\boldsymbol{e}_{\mathrm{x}}$ denotes the direction of the X-axis. Then $\sigma_{22}^{\text {aniso }} / \sigma_{11}^{\text {aniso }}$ is equal to $\tan ^{2}\left(\beta_{\text {act }}\right)$. 


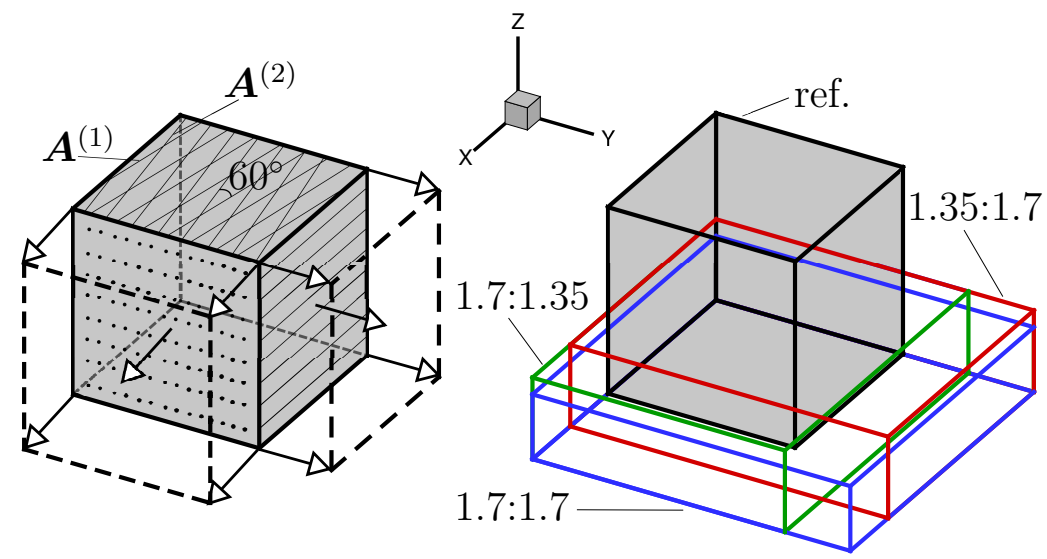

Figure 12: Biaxial tension test with two fiber families. The body is assumed to be incompressible with $F_{33}=1 /\left(F_{11} F_{22}\right)$
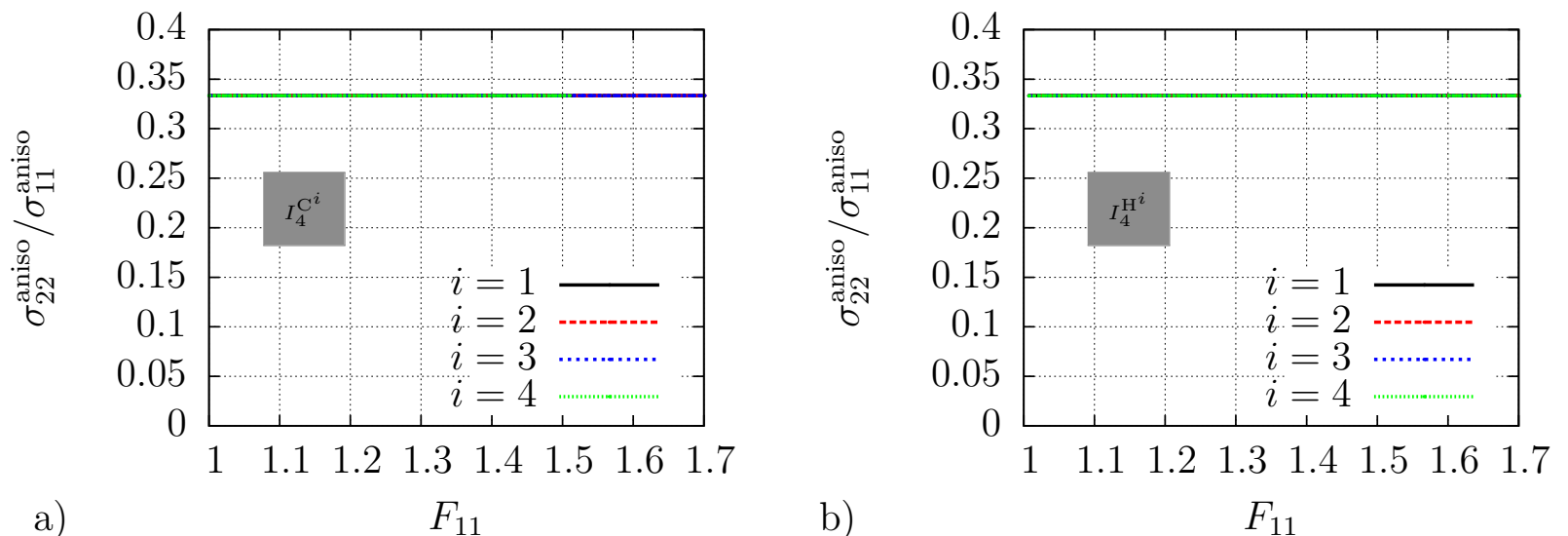

Figure 13: Evolution of the stress ratio for the equi-biaxial test with the stretch ratio $1.7: 1.7$ for a) the invariants $I_{4}^{\mathrm{C}^{i}}$ and b) the Hencky-type invariants $I_{4}^{\mathrm{H}^{i}}$ with the exponent $i=1,2,3,4$.
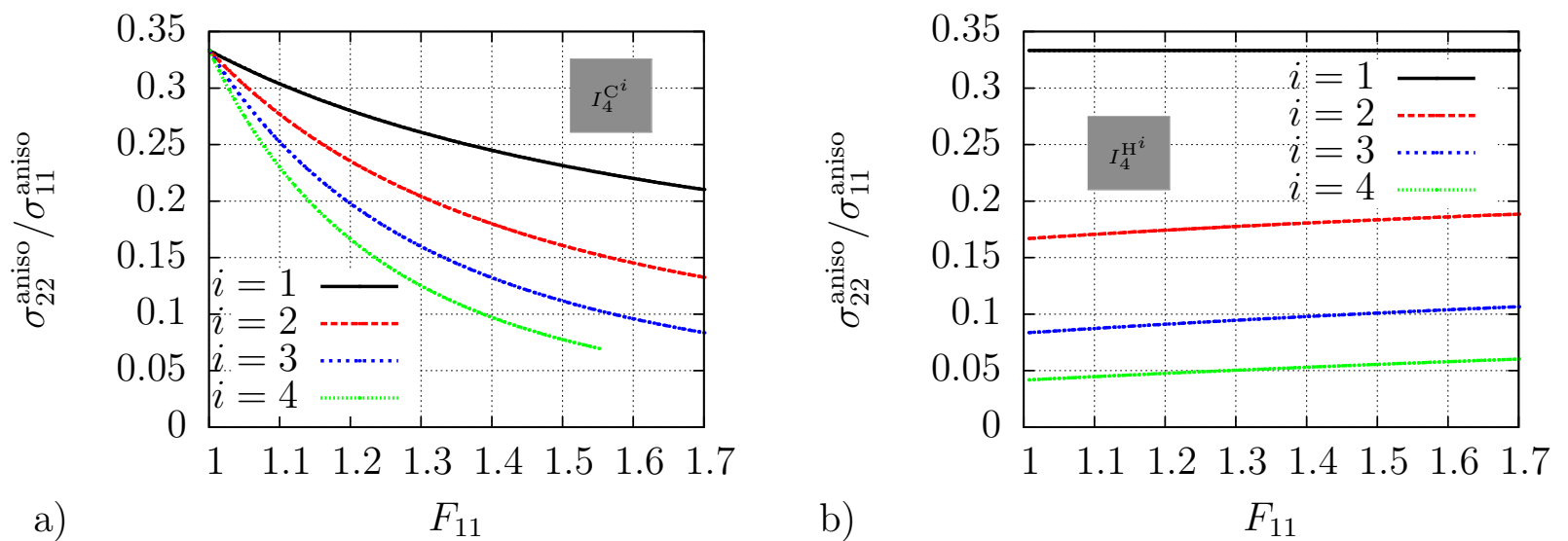

a)

$$
F_{11}
$$

b)

Figure 14: Evolution of the stress ratio for the biaxial test with the stretch ratio $1.7: 1.35$ for a) the invariants $I_{4}^{\mathrm{C}^{i}}$ and b) the Hencky-type invariants $I_{4}^{\mathrm{H}^{i}}$ with the exponent $i=1,2,3,4$. 

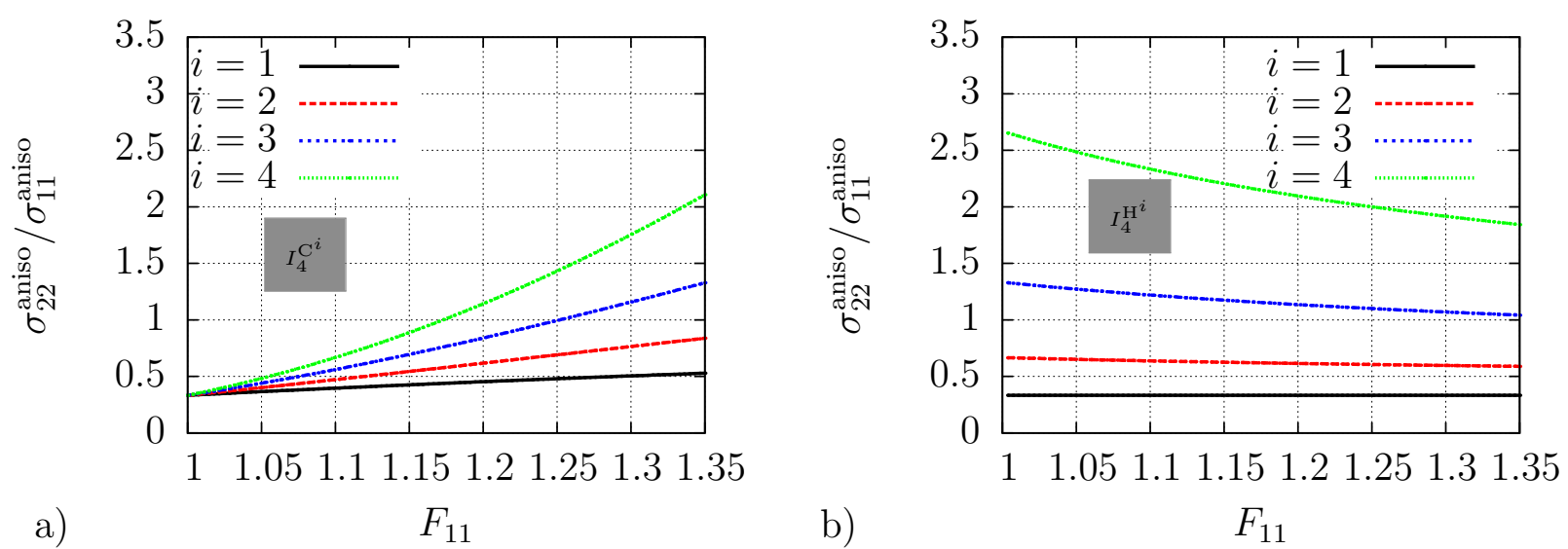

Figure 15: Evolution of the stress ratio for the biaxial test with the stretch ratio $1.35: 1.7$ for a) the invariants $I_{4}^{\mathrm{C}^{i}}$ and b) the Hencky-type invariants $I_{4}^{\mathrm{H}^{i}}$ with the exponent $i=1,2,3,4$.

4.3.4 Biaxial-tension-compression In order to complete the study of classical mechanical loading scenarios we consider a biaxial combined tension and compression test, assuming incompressibility. The problem is depicted in Fig. 16 and the fiber arrangement is identical to the previous example in section 4.3.3. While the stretches $\lambda_{x}$ and $\lambda_{y}$ are displacement driven, the principal stretch results form the incompressibility condition $\lambda_{x} \lambda_{y} \lambda_{z}=1$.

The fiber invariants, see Fig. 17, are identical for both fiber families and the evolution is comparable to the uniaxial case. In Fig. 18, again the evolution of the stress ratio is plotted, for both invariant sets. They appear to be quite different. For $I_{4}^{\mathrm{C}^{\mathrm{i}}}$ the stress ratio is approaching zero for infinite strains. For $I_{4}^{\mathrm{H}^{1}}$ we obtain a constant line as it was already the case in the biaxial tension test. If $i$ in $I_{4}^{\mathrm{H}^{\mathrm{i}}}$ is an even number $\sigma_{22}$ becomes negative, thus the stress ratio becomes negative.

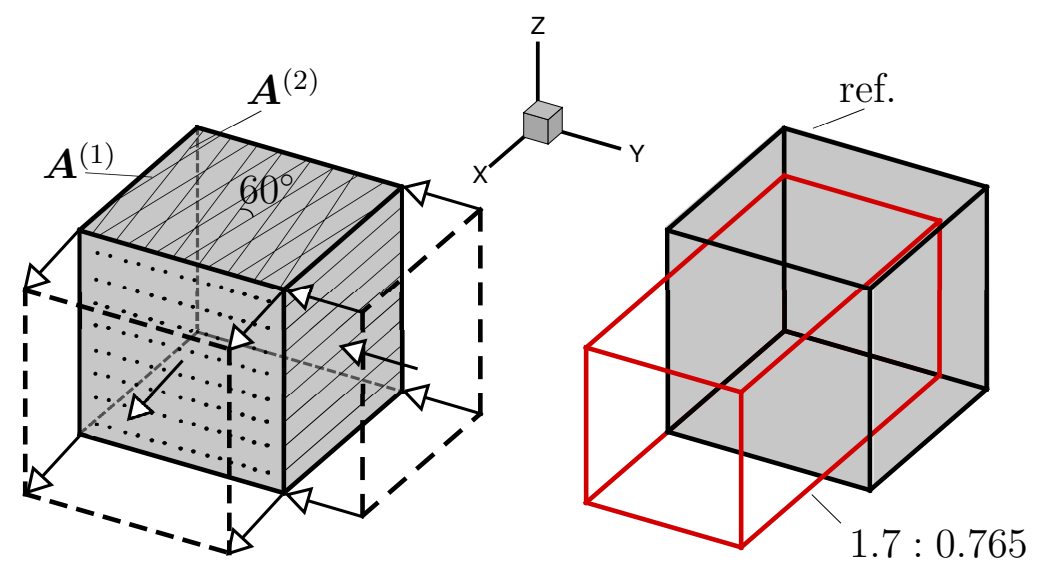

Figure 16: Biaxial combined compression and tension test, where the preferred direction and the loading are aligned. The body is assumed to be incompressible with $F_{33}=$ $1 /\left(F_{11} F_{22}\right)$ and $F_{33}=F_{22}$. 


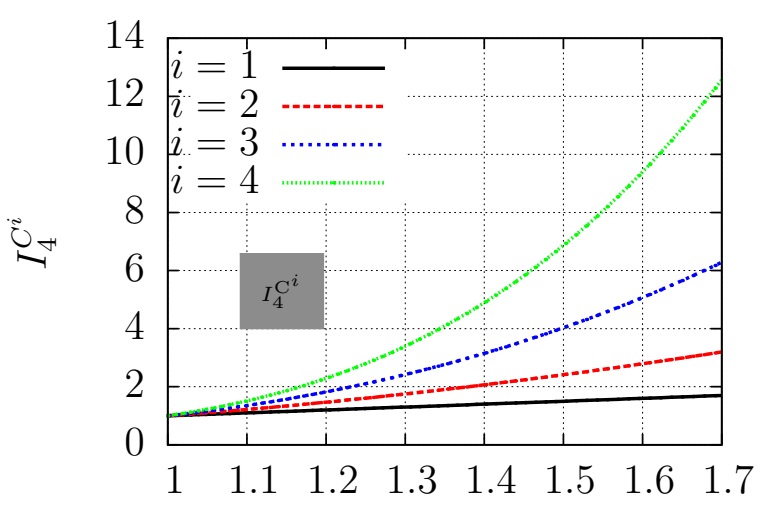

a) $F_{11}$

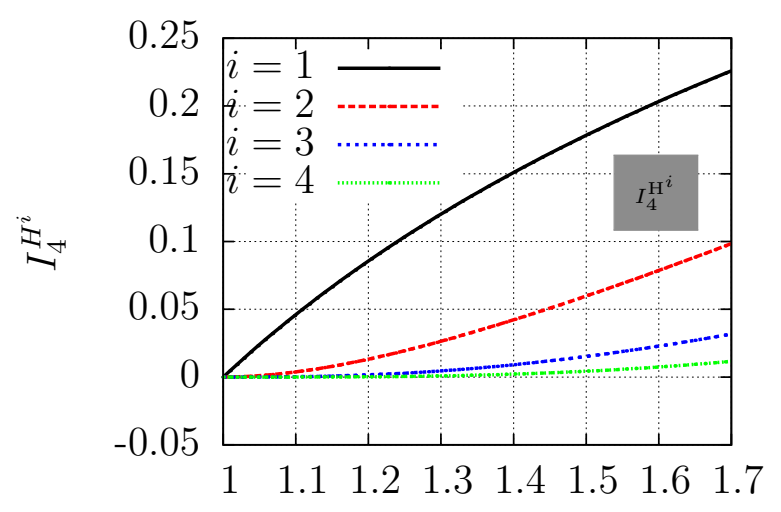

b)

$F_{11}$

Figure 17: Evolution of the anisotropic invariants a) $I_{4}^{\mathrm{C}^{i}}$ and b) $I_{4}^{\mathrm{H}^{i}}$ for the exponent $i=1,2,3,4$ under biaxial-tension-compression.

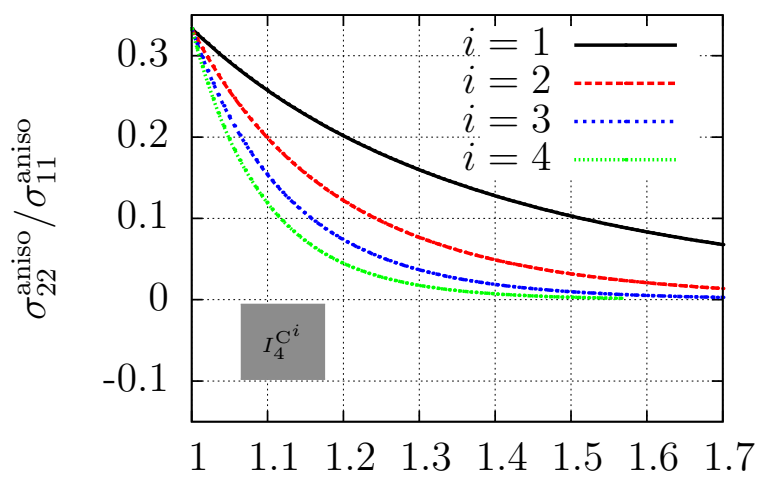

a) $F_{11}$

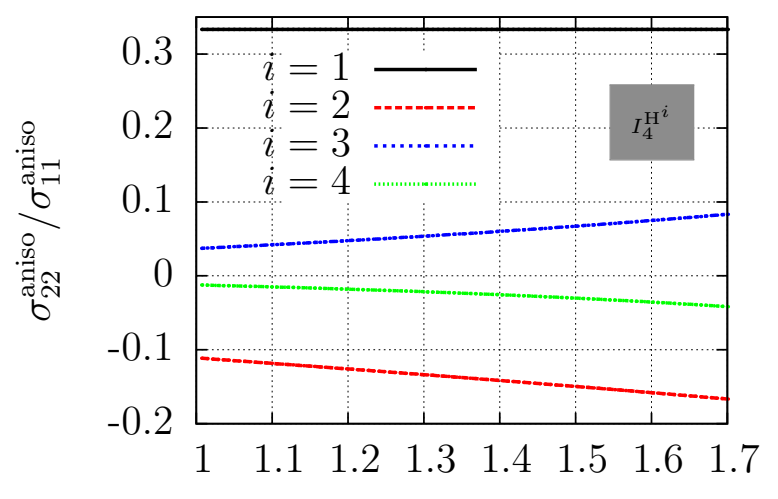

b)

$F_{11}$

Figure 18: Evolution of the stress ratio for the biaxial-tension-compression test for a) the invariants $I_{4}^{\mathrm{C}^{i}}$ and b) the Hencky-type invariants $I_{4}^{\mathrm{H}^{i}}$ with the exponent $i=1,2,3,4$.

\subsection{Adaption to biological soft tissues}

Soft biological tissues as they occur in arterial walls have an anisotropic material behavior. In biomechanical applications we often assume that the material behaves nearlyincompressible.

The widely used anisotropic strain energy introduced in Holzapfel et al. [30] is given by

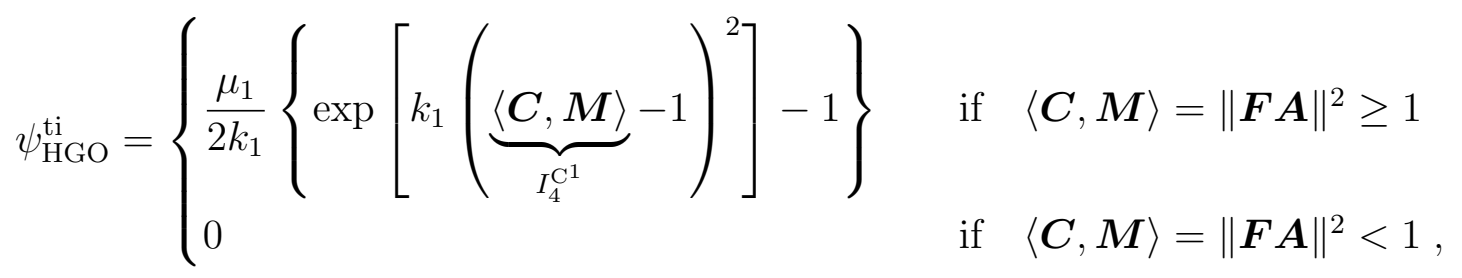

with the material parameters $\mu_{1}$ and $k_{1}$. Recall from Eq. (26), that $I_{4}^{\mathrm{C}^{1}}=\langle\boldsymbol{C}, \boldsymbol{M}\rangle$. The exponential function incorporated in the latter equation captures the material stiffening in the high strain domain, caused by the fiber elongation. The case distinction for the quadratic fiber elongation $\langle\boldsymbol{C}, \boldsymbol{M}\rangle=\|\boldsymbol{F} \boldsymbol{A}\|^{2}$ in Eq. (61) aims to prevent the fibers from inducing stiffness under compression. For further use below it is possible to rewrite the 
switching criterion for which the anisotropic fiber contribution is neglected, as

$$
\langle\boldsymbol{C}-\mathbf{1}, \boldsymbol{M}\rangle=\langle\boldsymbol{C}, \boldsymbol{M}\rangle-1<0 \text {. }
$$

When using anisotropic logarithmic invariants it seems natural to use criteria to switch off the compression regime which are themselves defined in terms of logarithmic invariants. However, as will be shown in the following, the case distinction for different anisotropic invariants (introduced in Eq. (26)) will lead to considerable differences.

Let us consider the right Cauchy-Green tensor

$$
\begin{aligned}
& \boldsymbol{C}=\sum_{k=1}^{3} \hat{\lambda}_{k} \boldsymbol{N}_{k} \otimes \boldsymbol{N}_{k}, \quad \text { with } \\
& \hat{\lambda}_{1}=0.9, \quad \hat{\lambda}_{2}=1.65, \quad \hat{\lambda}_{3}=\frac{1}{\hat{\lambda}_{1} \hat{\lambda}_{2}} \quad \text { and } \\
& \boldsymbol{N}_{1}=\left(\begin{array}{l}
1 \\
0 \\
0
\end{array}\right), \quad \boldsymbol{N}_{2}=\left(\begin{array}{l}
0 \\
1 \\
0
\end{array}\right), \quad \mathbf{N}_{3}=\left(\begin{array}{l}
0 \\
0 \\
1
\end{array}\right)
\end{aligned}
$$

represented in the spectral decomposition. The considered deformation is incompressible, i.e. $\operatorname{det} \boldsymbol{C}=1$. The set of all possible preferred directions $\boldsymbol{A}$ may be expressed with help of the spherical coordinates $(r, \phi, \theta)$ and

$$
\begin{aligned}
& x=r \sin \theta \cos \phi, \\
& y=r \sin \theta \sin \phi, \\
& z=r \cos \phi .
\end{aligned}
$$

Here, $r$ denotes the radius, $\phi \in[-\pi, \pi]$ the polar angle and $\theta \in[0, \pi]$ the azimuthal angle. Since $\|\boldsymbol{A}\|=1$, we choose $r=1$ and consequently

$$
\boldsymbol{A}=\left(\begin{array}{c}
\sin \theta \cos \phi \\
\sin \theta \sin \phi \\
\cos \phi
\end{array}\right) \text {. }
$$

Then the anisotropic invariants can be computed from

$$
\begin{aligned}
\left\langle\boldsymbol{C}^{i}, \boldsymbol{M}\right\rangle & =\widehat{\lambda}_{1}^{i} \cos ^{2} \theta_{1}+\widehat{\lambda}_{2}^{i} \cos ^{2} \theta_{2}+\widehat{\lambda}_{3}^{i} \cos ^{2} \theta_{3} \quad \text { and } \\
\left\langle(\log \boldsymbol{U})^{i}, \boldsymbol{M}\right\rangle & =\left(\frac{1}{2} \log \widehat{\lambda}_{1}\right)^{i} \cos ^{2} \theta_{1}+\left(\frac{1}{2} \log \widehat{\lambda}_{2}\right)^{i} \cos ^{2} \theta_{2}+\left(\frac{1}{2} \log \widehat{\lambda}_{3}\right)^{i} \cos ^{2} \theta_{3},
\end{aligned}
$$

where

$$
\cos \theta_{1}=\left\langle\boldsymbol{A}, \boldsymbol{N}_{1}\right\rangle \quad \cos \theta_{2}=\left\langle\boldsymbol{A}, \boldsymbol{N}_{2}\right\rangle \quad \cos \theta_{3}=\left\langle\boldsymbol{A}, \boldsymbol{N}_{3}\right\rangle .
$$

The invariants, depending on the fiber orientation $\boldsymbol{A}(x, y, z)$, are plotted in Fig. 19. While the principal distributions are similar for different exponents of $\boldsymbol{C}$, it appears that the distributions are very different for even and odd exponents $i$ for the logarithmic invariants, depending on $\log \boldsymbol{U}$. Please note that a change in the eigenvectors $\boldsymbol{N}_{k} \mid k=1,2,3$ would merely lead to a rotation of the plotted sphere around the eigenvector base. Therefore, in this scheme the eigenvalues remain as the only predefined variables. As our main goal 
is to exclude the compression state from the anisotropic material response to induce any stiffening we consider the sign of the invariants $\left\langle(\log \boldsymbol{U})^{i}, \boldsymbol{M}\right\rangle$ and $\left\langle\boldsymbol{C}^{i}, \boldsymbol{M}\right\rangle-1$ as the determining criterion. The corresponding distributions are plotted in Fig. 20 over the azimuthal and polar angle, which are sufficient in order to uniquely define the fiber orientation. In that sense the black area representing negative values labels the fiber directions for which the fiber response will be switched off. On the other hand the red areas of positive values cover the fiber directions for which the anisotropic strain energy function is switched on. For the invariants depending on $\boldsymbol{C}^{i}$ the area of positive values will increase with the exponent $i$ and become more elliptic. The plots for $\operatorname{sign}(\langle\boldsymbol{C}, \boldsymbol{M}\rangle-1)$ and $\operatorname{sign}(\langle\log \boldsymbol{U}, \boldsymbol{M}\rangle)$ are generally similar. However, with help of Eq. (64) it appears that

$$
\begin{aligned}
\langle\boldsymbol{C}, \boldsymbol{M}\rangle-1-\langle\log \boldsymbol{C}, \boldsymbol{M}\rangle & =\langle\boldsymbol{C}-\mathbf{1}, \boldsymbol{M}\rangle-\langle\log \boldsymbol{C}, \boldsymbol{M}\rangle \\
& =\sum_{k}^{3}(\underbrace{\widehat{\lambda}_{k}-1-\log \widehat{\lambda}_{k}}_{\geq 0}) \underbrace{\cos ^{2} \theta_{k}}_{\geq 0} \geq 0
\end{aligned}
$$

and therefore

$$
\langle\boldsymbol{C}, \boldsymbol{M}\rangle-1 \geq\langle\log \boldsymbol{C}, \boldsymbol{M}\rangle
$$

Note that $\cos ^{2} \theta_{j}$ is the same for each invariant, because the eigenvectors $\boldsymbol{N}_{j}$ for each considered strain measure are the same. It immediately follows that

$$
[\langle\boldsymbol{C}, \boldsymbol{M}\rangle-1] \geq \frac{1}{2}\langle\log \boldsymbol{C}, \boldsymbol{M}\rangle=\langle\log \boldsymbol{U}, \boldsymbol{M}\rangle \quad \text { for } \quad\langle\boldsymbol{C}, \boldsymbol{M}\rangle \geq 1
$$

That means there exists a transition zone, where $\langle\boldsymbol{C}, \boldsymbol{M}\rangle-1>0$, but $\langle(\log \boldsymbol{U}), \boldsymbol{M}\rangle<0$. In other words, one may conclude that it is possible that the fiber direction may be stretched and still the criterion $\langle(\log \boldsymbol{U}), \boldsymbol{M}\rangle$ will switch off the anisotropic response. This effect may be favorably used when it is assumed that initially crimped fibers don't exhibit significant stiffness until they are straightened out. However, whenever the fiber direction is compressed, anisotropic material response is precluded for the discussed logarithmic transversely isotropic strain measure based on the criterion $\langle\log \boldsymbol{U}, \boldsymbol{M}\rangle<0$. Thus, there is no anisotropic stiffening under compression.

For $\left\langle(\log \boldsymbol{U})^{2}, \boldsymbol{M}\right\rangle$ and $\left\langle(\log \boldsymbol{U})^{4}, \boldsymbol{M}\right\rangle$ (and also any other even exponent) the invariants will always have a positive sign, since both, the structural tensor $\boldsymbol{M}$ as well as $(\log \boldsymbol{U})^{2}$ and $(\log \boldsymbol{U})^{4}$ are positive semidefinite. Consequently, these invariants may not serve as a switching criterion.

In the light of the previous considerations, we may formulate the generalized strain energy 

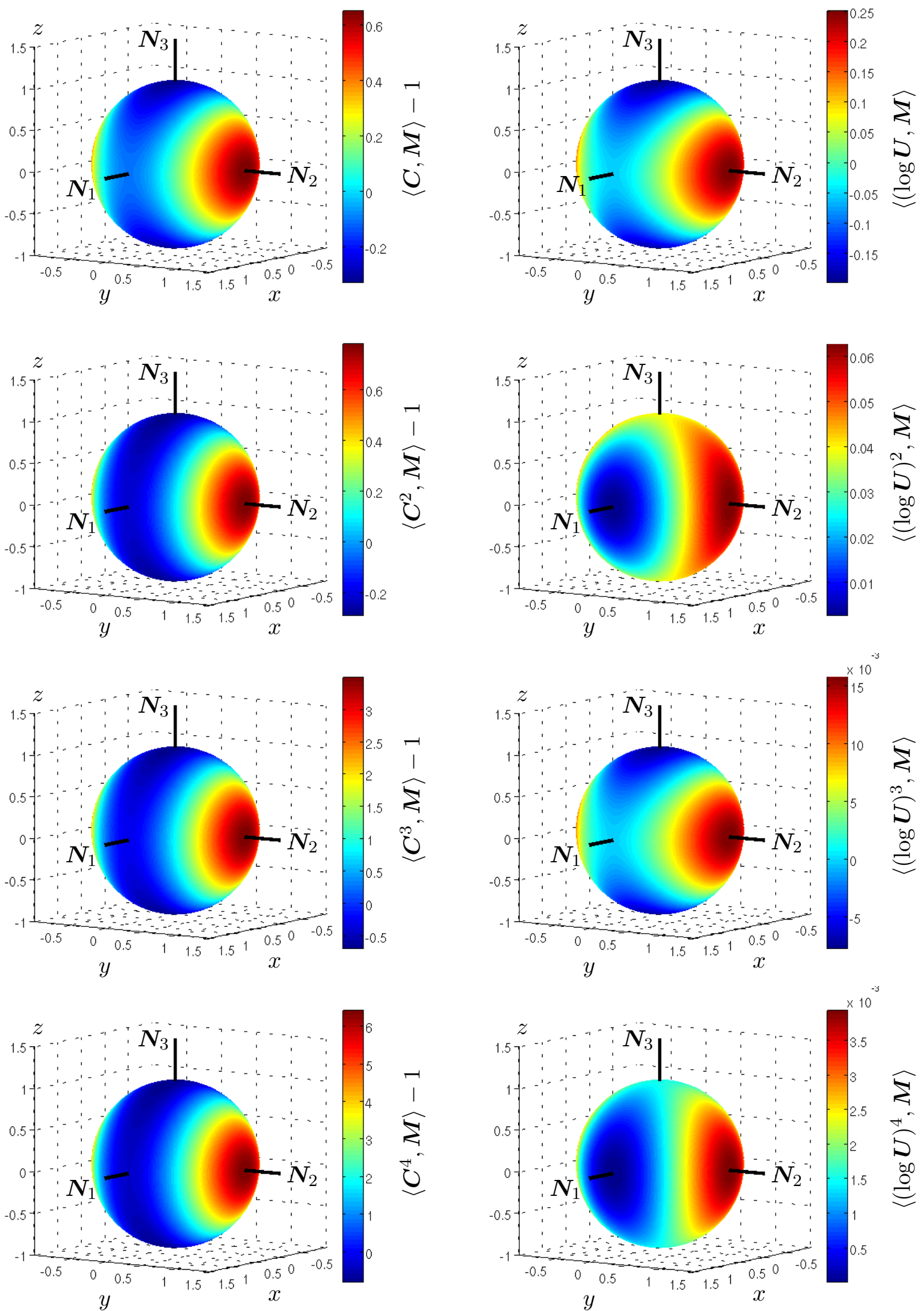

Figure 19: Contour plot of different transversely isotropic invariants for a specific choice of eigenvalues and eigenvectors. The coordinates $(x, y, z)$ define the preferred direction $\boldsymbol{A}$. 

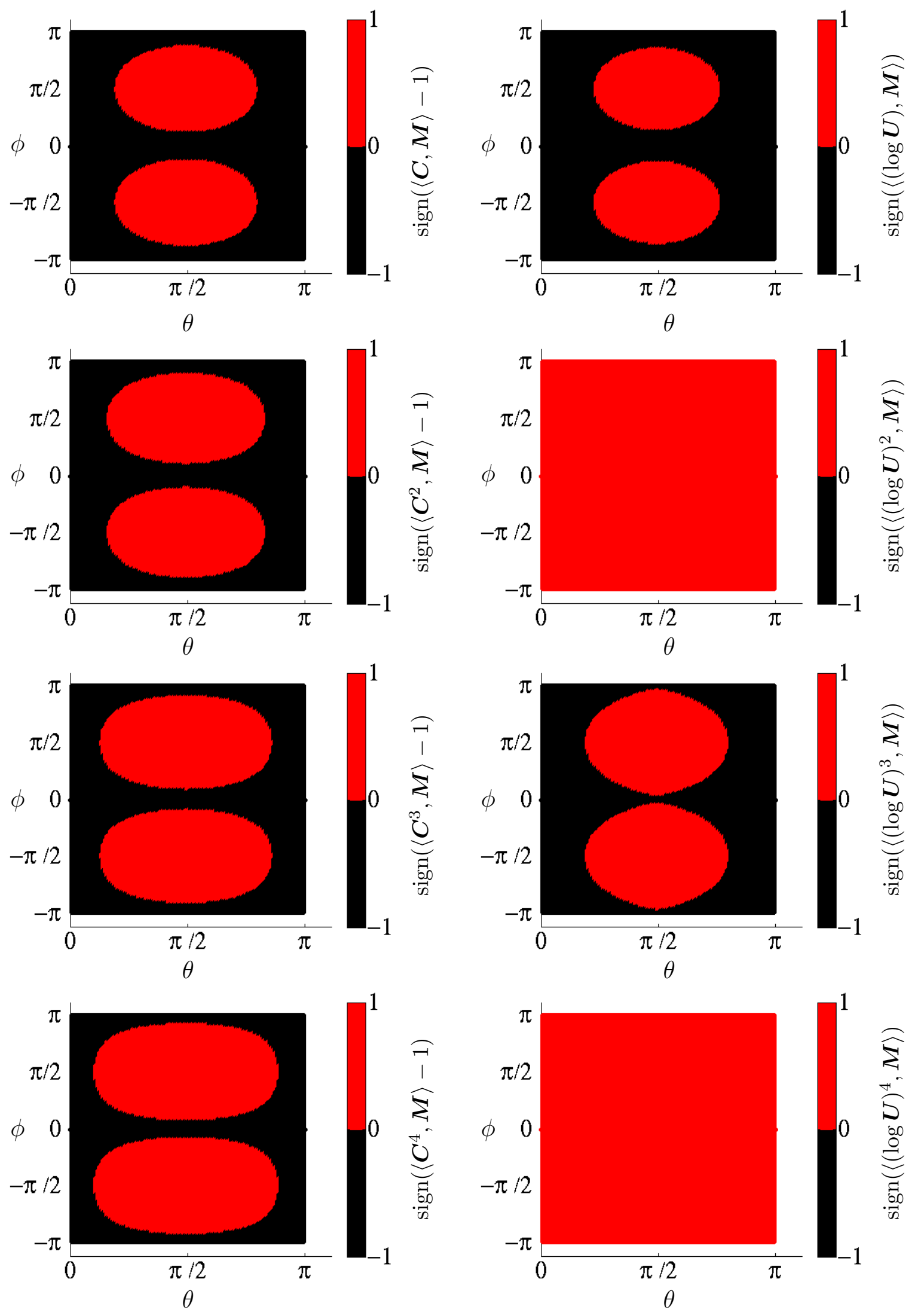

Figure 20: Plot of the sign of the invariants plotted in Fig. 19 over the spherical coordinates. The boundary between positive and negative values marks the transition zone of a possible case distinction. 
functions

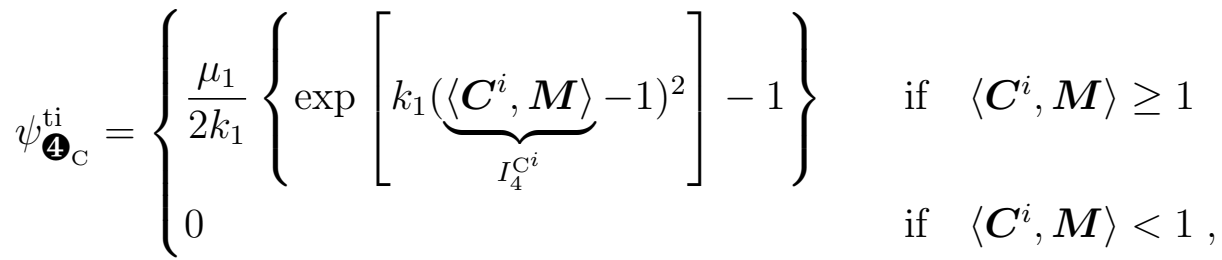

$$
\begin{aligned}
& \psi_{\boldsymbol{\sigma}_{\mathrm{H}}}^{\mathrm{ti}}=\left\{\frac{\mu_{1}}{2 k_{1}}\left\{\exp [k_{1} \underbrace{\langle\log \boldsymbol{U}, \boldsymbol{M}\rangle}_{I_{4}^{\mathrm{H}^{1}}} \underbrace{\left\langle(\log \boldsymbol{U})^{i}, \boldsymbol{M}\right\rangle^{2}}_{I_{4}^{\mathrm{H}^{i}}}]-1\right\} \quad \begin{array}{rl}
\text { if } \quad\langle\log \boldsymbol{U}, \boldsymbol{M}\rangle \geq 0 \\
& \text { if }\langle\log \boldsymbol{U}, \boldsymbol{M}\rangle<0,
\end{array}\right.
\end{aligned}
$$

in terms of the anisotropic invariants $I_{4}^{\mathrm{C}^{i}} \mid i=1,2,3,4$ and $I_{4}^{\mathrm{H}^{i}} \mid i=1,2,3,4$, defined in Eq. (26). In Eq. (68) the value of $\varepsilon$ is to be chosen positive and close to zero. The incorporation of $\langle\log \boldsymbol{U}, \boldsymbol{M}\rangle^{\varepsilon}$ in the free energy function prevents jumps in the stresses at the switch over point. Alternatively one may also incorporate the criterion $(\langle\boldsymbol{C}, \boldsymbol{M}\rangle-1)^{\varepsilon}$ and formulate the case distinction based on the quadratic fiber stretch $\langle\boldsymbol{C}, \boldsymbol{M}\rangle$. As it is shown below a continuous material tangent is also ensured.

Case distinctions, like in Eq. (67) and Eq. 68 may generally lead to discontinuous functions. In order to avoid this it is evident that the stresses and the tangent must become zero at each switch-point of the chosen criterion. Indeed one can show that

$$
\begin{array}{ccc}
\frac{\partial \psi_{\boldsymbol{\Theta}_{\mathrm{C}}^{\mathrm{ti}}}^{\mathrm{ti}}=\mathbf{0}}{\partial \boldsymbol{C}} \quad \text { if } & \left\langle\boldsymbol{C}^{i}, \boldsymbol{M}\right\rangle=1, \\
\frac{\partial \psi_{\boldsymbol{G}_{\mathrm{H}}}^{\mathrm{ti}}}{\partial \boldsymbol{C}}=\mathbf{0} & \text { if } & \langle\log \boldsymbol{U}, \boldsymbol{M}\rangle=0,
\end{array}
$$

such that no jumps in the stresses at the switchover points are possible. Only $\psi_{\mathbf{4}_{\mathrm{C}}}^{\mathrm{ti}}$ of the introduced strain energy calsses misses continuity, since

$$
\begin{array}{llc}
\frac{\partial^{2} \psi_{\boldsymbol{\mathbf { 4 }}_{\mathrm{C}}^{\mathrm{C}}}^{\mathrm{ti}} \neq \mathbf{0}}{\partial \boldsymbol{C} \partial \boldsymbol{C}} \quad \text { if } & \left\langle\boldsymbol{C}^{i}, \boldsymbol{M}\right\rangle=1, \\
\frac{\partial^{2} \psi_{\boldsymbol{\sigma}_{\mathrm{H}}}^{\mathrm{ti}}=\mathbf{0}}{\partial \boldsymbol{C} \partial \boldsymbol{C}} \quad \text { if } & \langle\log \boldsymbol{U}, \boldsymbol{M}\rangle=0,
\end{array}
$$

The material tangent of the function $\psi_{\mathbf{4}_{\mathrm{C}}}^{\mathrm{ti}}$ at the point $\boldsymbol{C}=\mathbf{1}$ in the reference configuration with a structural tensor $\boldsymbol{M}=\operatorname{diag}(0,0,1)$,

$$
\left.\mathbb{C}\right|_{\boldsymbol{C}=\mathbf{1}}\left(\psi_{\boldsymbol{\Theta}_{\mathrm{C}}}^{\mathrm{ti}}\left(I_{4}^{\mathrm{C}^{i}} \mid i=1,2,3,4\right)\right)=\left(\begin{array}{cccccc}
0 & 0 & 0 & 0 & 0 & 0 \\
0 & 0 & 0 & 0 & 0 & 0 \\
0 & 0 & 4 i^{2} \mu_{1} & 0 & 0 & 0 \\
0 & 0 & 0 & 0 & 0 & 0 \\
0 & 0 & 0 & 0 & 0 & 0 \\
0 & 0 & 0 & 0 & 0 & 0
\end{array}\right),
$$

is already different from $\mathbf{0}$ and therefore violates the continuity requirement. For the class $\psi_{\boldsymbol{\sigma}_{\mathrm{H}}}^{\mathrm{ti}}$ we find that $\left.\mathbb{C}\right|_{\boldsymbol{C}=\mathbf{1}}=\mathbf{0}$. 


\section{Parameter adjustment}

\subsection{Parameter identification for soft biological tissues}

In the following the proposed transversely isotropic Hencky models are adjusted to the test data provided in Holzapfel [29]. There, a human, abdominal aorta from a human cadaver was tested. The donor was female, 80 years old and suffering from congestive cardiomyopathy. Arterial stripes were excised for two material layers, as indicated in Fig. 21a). Therefore, we introduce the orthonormal coordinate system depending on the circumferential direction $\boldsymbol{N}_{\varphi}$, the axial direction $\boldsymbol{N}_{z}$ and the radial direction $\boldsymbol{N}_{r}$. For each of the layers the tissue was stretched in either circumferential (see Fig. 21b)) or axial (see Fig. 21c)) direction.

In an incompressible uniaxial tension test with two fiber families orientated in the $\boldsymbol{N}_{\varphi}-\boldsymbol{N}_{z}$ plane, we may write the tensors

$$
\begin{aligned}
\boldsymbol{F} & =\boldsymbol{U}=\left(\begin{array}{ccc}
\sqrt{\widehat{\lambda}_{1}} & 0 & 0 \\
0 & \sqrt{\widehat{\lambda}_{2}} & 0 \\
0 & 0 & \sqrt{\frac{1}{\widehat{\lambda}_{1} \widehat{\lambda}_{2}}}
\end{array}\right), \quad \boldsymbol{C}=\left(\begin{array}{ccc}
\widehat{\lambda}_{1} & 0 & 0 \\
0 & \widehat{\lambda}_{2} & 0 \\
0 & 0 & \frac{1}{\widehat{\lambda}_{1} \widehat{\lambda}_{2}}
\end{array}\right) \text { and } \\
\log \boldsymbol{U} & =\left(\begin{array}{ccc}
\frac{1}{2} \log \left(\widehat{\lambda}_{1}\right) & 0 & 0 \\
0 & \frac{1}{2} \log \left(\widehat{\lambda}_{2}\right) & 0 \\
0 & 0 & \frac{1}{2} \log \left(\frac{1}{\widehat{\lambda}_{1} \widehat{\lambda}_{2}}\right)
\end{array}\right) .
\end{aligned}
$$

Because of the incompressibility we have $\operatorname{tr}(\log \boldsymbol{U})=\log (\operatorname{det} \boldsymbol{U})=0$ and the second part of $W_{\mathrm{H}}$ and $W_{\mathrm{eH}}$ becomes automatically zero. Moreover, the structural tensors are

$$
\boldsymbol{M}_{(1)}=\left(\begin{array}{ccc}
c^{2} & -c s & 0 \\
-c s & s^{2} & 0 \\
0 & 0 & 0
\end{array}\right) \quad \text { and } \quad \boldsymbol{M}_{(2)}=\left(\begin{array}{ccc}
c^{2} & c s & 0 \\
c s & s^{2} & 0 \\
0 & 0 & 0
\end{array}\right)
$$

with $c=\cos \beta_{\mathrm{f}}$ and $s=\sin \beta_{\mathrm{f}}$ and therefore we have

$$
\begin{aligned}
I_{4}^{\mathrm{H}^{\mathrm{i}}} & =\left(\frac{1}{2} \log \left(\widehat{\lambda}_{1}\right)\right)^{\mathrm{i}}\left(\cos \beta_{\mathrm{f}}\right)^{2}+\left(\frac{1}{2} \log \left(\widehat{\lambda}_{2}\right)\right)^{\mathrm{i}}\left(\sin \beta_{\mathrm{f}}\right)^{2} \quad \text { and } \\
I_{4}^{\mathrm{C}^{\mathrm{i}}} & =\widehat{\lambda}_{1}^{\mathrm{i}}\left(\cos \beta_{\mathrm{f}}\right)^{2}+\widehat{\lambda}_{2}^{\mathrm{i}}\left(\sin \beta_{\mathrm{f}}\right)^{2}
\end{aligned}
$$

The angle $\beta_{\mathrm{f}}$ denotes the angle between each fiber and the local circumferential direction, while the angle between both fibers follows to $2 \beta_{\mathrm{f}}$. The second Piola-Kirchoff stresses in this case may be written as

$$
S_{11}=2 \frac{\partial \psi}{\partial \widehat{\lambda}_{1}}+2 \frac{p}{\widehat{\lambda}_{1}}, \quad S_{22}=2 \frac{\partial \psi}{\partial \widehat{\lambda}_{2}}+2 \frac{p}{\widehat{\lambda}_{2}}, \quad S_{33}=2 \frac{\partial \psi}{\partial \frac{1}{\widehat{\lambda}_{1} \widehat{\lambda}_{2}}}+2 \frac{p}{\widehat{\lambda}_{1} \widehat{\lambda}_{2}}
$$

The Lagrange multiplier $p$ is introduced in order to enforce the incompressibility and can directly be calculated with help of the requirement that $S_{22}$ must be equal to zero. The stretch $\widehat{\lambda}_{11}$ is known from the experiments and the remaining unknown $\widehat{\lambda}_{22}$ is iterated 
with help of Newton's method, making use of the requirement that also $S_{33}$ must be equal to zero:

$$
S_{33}\left(\widehat{\lambda}_{2}\right) \stackrel{!}{=} 0 \quad \Rightarrow \widehat{\lambda}_{2}^{n+1}=\widehat{\lambda}_{2}^{n}-\frac{S_{33}\left(\widehat{\lambda}_{2}^{n}\right)}{\operatorname{Lin} S_{33}\left(\widehat{\lambda}_{2}^{n}\right)}, \quad \text { with } \quad \operatorname{Lin} S_{33}\left(\widehat{\lambda}_{2}^{n}\right)=\frac{\partial S_{33}\left(\widehat{\lambda}_{2}^{n}\right)}{\partial \widehat{\lambda}_{2}^{n}}
$$

The parameter fitting was performed with help of a Sequential Quadratic Programming (SQP) algorithm for nonlinear numerical constrained optimization problems. The gradient needed for the optimization procedure is calculated based on a finite difference scheme in conjunction with the above described Newton iteration. The objective function

$$
f^{\mathrm{obj}}(\boldsymbol{\alpha}):=\sum_{\mathrm{e}=1}^{n_{\text {exp }}} \sqrt{\frac{1}{n_{\mathrm{mp}}} \sum_{i=1}^{n_{\mathrm{mp}}}\left(\frac{\sigma_{e}^{\exp }\left(\widehat{\lambda}_{k}^{i}\right)-\sigma^{\operatorname{sim}}\left(\widehat{\lambda}_{k}^{i}, \boldsymbol{\alpha}\right)}{\max \left(\sigma_{e}^{\exp }\right)}\right)^{2}}
$$

is utilized as the optimization criterion. Here $n_{\exp }$ and $n_{\mathrm{mp}}$ denote the number of experiments to be fitted and the number of specific measuring points to be evaluated. The predefined amount of stretch associated to each measuring point $i$ is labeled with $\widehat{\lambda}_{k}^{i}$. The simulated Cauchy stresses $\sigma^{\text {sim }}$ and the error $f^{\text {obj }}$ are dependent on the chosen material parameter set contained in the field $\boldsymbol{\alpha}$.
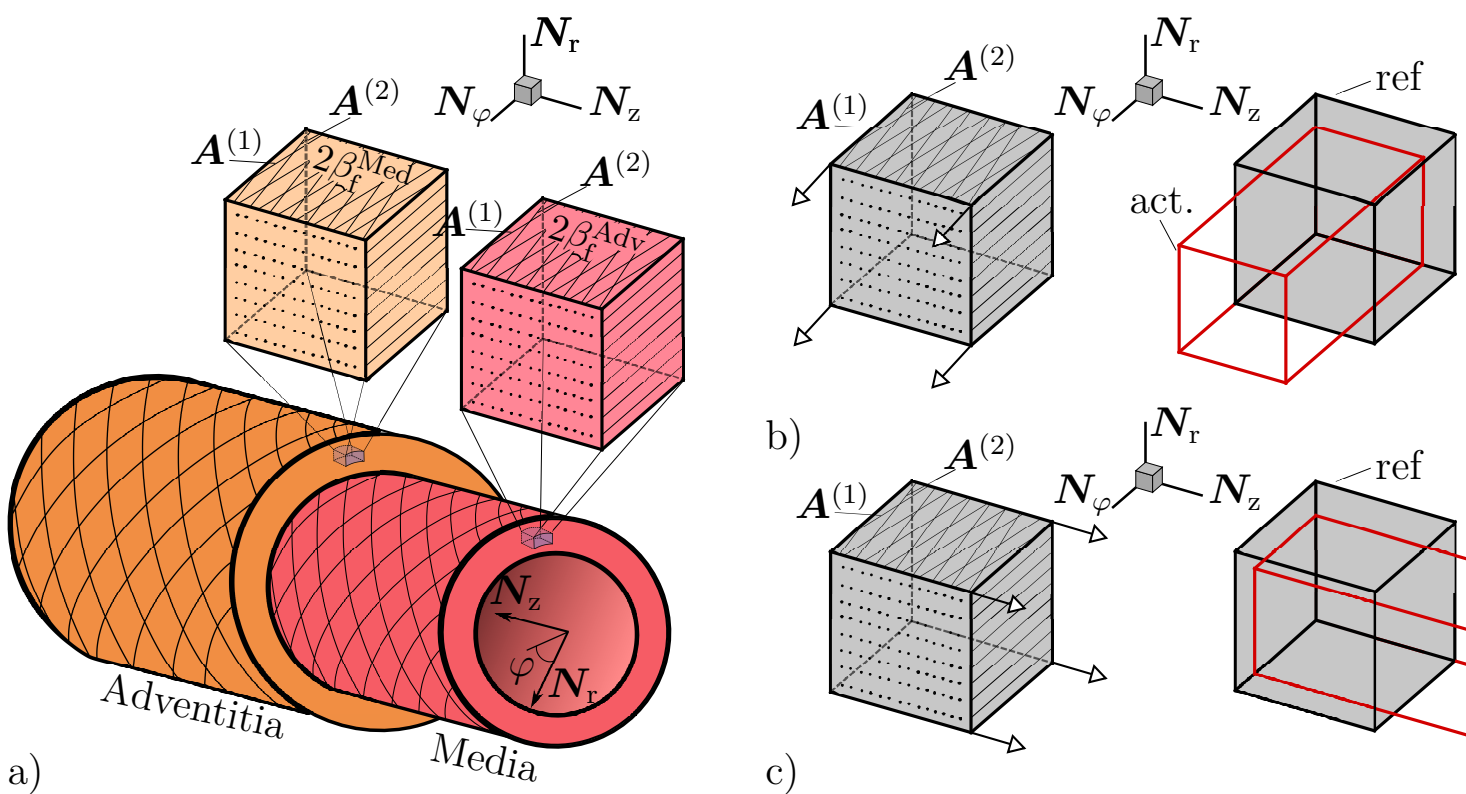

b)

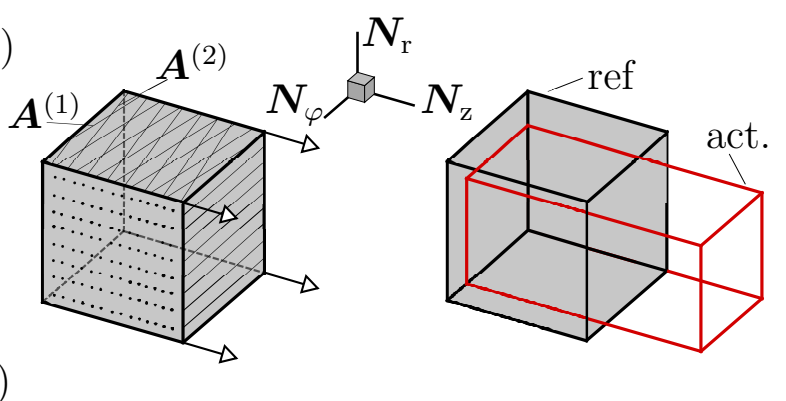

Figure 21: a) Excised tissue samples, b) uniaxial tension test in circumferential and c) in axial direction.

The values of the shear modulus $\mu$ for the isotropic response will be directly estimated from the experiments as the average of the circumferential and axial stiffness. This is possible since the initial tangent $\left.\mathbb{C}\right|_{\boldsymbol{C}=\mathbf{1}}$ of all adjusted strain energies is solely determined by $\mu$ and will be independent of the anisotropic response. Then considering that

$$
\Delta \boldsymbol{S}=\left.\mathbb{C}\right|_{C=1}: \frac{1}{2} \Delta \boldsymbol{C},
$$

and $\mathbb{C}$ being a function only of $\mu$ in the reference configuration, the value of $\mu$ can be directly computed for the first measuring point $\boldsymbol{C}\left(\hat{\lambda}_{k}^{1}\right)$. This is done separately for both 
directions and the mean value is taken as the estimated parameter. However, $\mu$ could as well be considered as a parameter to be included in the parameter set $\boldsymbol{\alpha}$ in Eq. (73), which is to be optimized. Due to the assumed incompressibility it is impossible to estimate the bulk modulus $\kappa$. From the numerical point of view the bulk modulus may act like a penalty constraint to enforce quasi incompressibility in more complex computations.

\subsection{Media}

The results of the parameter adjustment of the Media are given in Table 3 for 8 different models and the corresponding stress-strain curves are plotted in Fig. 22. These models result from the combination of the two isotropic strain energy functions in Eq. (29) and Eq. (35) and the proposed transversely isotropic functions in Eq. (68). The exponent $\varepsilon$ for the switchover criterion is set to 0.1 for all functions. The fiber angle $\beta_{\mathrm{f}}$ between the fiber direction and the circumferential direction was part of the optimization, while the parameters $\kappa$ and $\hat{k}$ were excluded from the optimization, due to the above assumed quasi-incompressibility. The isotropic shear modulus was directly estimated from the experiments and was also not optimized.

Apparently, model 1 and 5 fail to accurately fit the experimental data which is evident in the large values of the objective function $f^{\text {obj }}$. When considering the exponentiated Hencky energy $W_{\mathrm{eH}}$ instead of the classical isotropic energy $W_{\mathrm{H}}$ the fit quality is substantially improved for models 2 and 6 . Nevertheless, one has to admit that the associated fiber angle becomes unsatisfactory small, which does not seem to be reasonable. The small fiber angle is also accompanied by the effect that the isotropic material response in this case is highly nonlinear. The functions which are based on invariants with even exponents generally seem to perform better, independent of the chosen isotropic strain energy function. In Holzapfel [29], a mean angle of $37.5^{\circ}$ was reported. The optimized fiber angles of model 3 , 4,7 and 8 are of this order of magnitude. When $W_{\mathrm{eH}}$ is used the error generally becomes slightly smaller, since one additional parameter is available.

\begin{tabular}{|c|c|c|c|c|c|c|c|}
\hline & $\psi$ in - & $\mu$ in $\mathrm{kPa}$ & $k$ in - & $\mu_{1}$ in $\mathrm{kPa}$ & $k_{1}$ in - & $\beta_{\mathrm{f}}$ in ${ }^{\circ}$ & $f^{\text {obj }}$ \\
\hline 1 & $W_{\mathrm{H}}+\sum_{a=1}^{2} \psi_{\boldsymbol{\Theta}_{H}}^{\mathrm{ti}}\left(I_{4_{(a)}}^{\mathrm{H}^{1}}\right)$ & 31.16 & - & 0.0001 & 948.81 & 25.36 & 0.426 \\
\hline 2 & $W_{\mathrm{eH}}+\sum_{a=1}^{2} \psi_{\mathbf{\varphi}_{H}}^{\mathrm{ti}}\left(I_{4_{(a)}}^{\mathrm{H}^{1}}\right)$ & 31.16 & 10.54 & 0.50 & 107.94 & 0.73 & 0.071 \\
\hline 3 & $W_{\mathrm{H}}+\sum_{a=1}^{2} \psi_{\boldsymbol{\Phi}_{H}}^{\mathrm{ti}}\left(I_{4_{(a)}}^{\mathrm{H}^{2}}\right)$ & 31.16 & - & 1204.86 & 1599.53 & 41.24 & 0.046 \\
\hline 4 & $W_{\mathrm{eH}}+\sum_{a=1}^{2} \psi_{\mathbf{\varphi}_{H}}^{\mathrm{ti}}\left(I_{4_{(a)}}^{\mathrm{H}^{2}}\right)$ & 31.16 & 3.38 & 726.09 & 1848.66 & 40.68 & 0.044 \\
\hline 5 & $W_{\mathrm{H}}+\sum_{a=1}^{2} \psi_{\boldsymbol{\varphi}_{H}}^{\mathrm{ti}}\left(I_{4_{(a)}}^{\mathrm{H}^{3}}\right)$ & 31.16 & - & 11677.63 & 3112.51 & 0.0001 & 0.386 \\
\hline 6 & $W_{\mathrm{eH}}+\sum_{a=1}^{2} \psi_{\mathbf{\varphi}_{H}}^{\mathrm{ti}}\left(I_{4_{(a)}}^{\mathrm{H}^{3}}\right)$ & 31.16 & 10.54 & 5033.61 & 17685.18 & 28.74 & 0.071 \\
\hline 7 & $W_{\mathrm{H}}+\sum_{a=1}^{2} \psi_{\boldsymbol{\varphi}_{H}}^{\mathrm{ti}}\left(I_{4_{(a)}}^{\mathrm{H}^{4}}\right)$ & 31.16 & - & 591428.36 & 51778.23 & 38.49 & 0.100 \\
\hline 8 & $W_{\mathrm{eH}}+\sum_{a=1}^{2} \psi_{\boldsymbol{\Phi}_{H}}^{\mathrm{ti}}\left(I_{4_{(a)}}^{\mathrm{H}^{4}}\right)$ & 31.16 & 7.56 & 232287.68 & 174224.46 & 36.86 & 0.052 \\
\hline
\end{tabular}

Table 3: Adjusted parameter sets of the Media. 

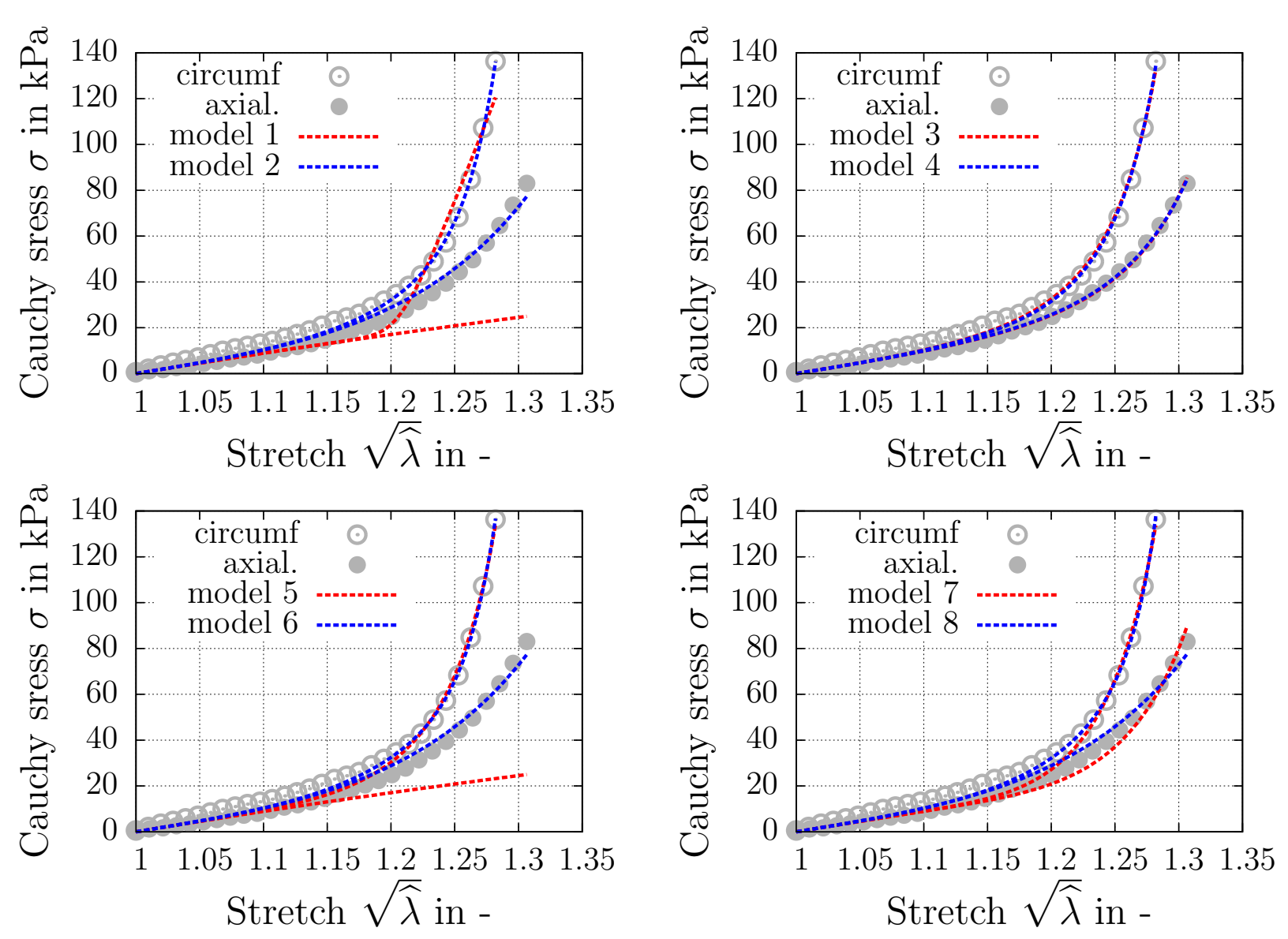

Figure 22: Adjusted stress-strain curves for the media for model 1-8.

\subsection{Adventitia}

The case distinction in the isotropic response for the parameter fitting is neglected for the Adventitia. Only four models are tested in the following. The optimized parameters are shown in Table 4 and the corresponding stress-strain curves are given in Fig. 23. Please note that in this case the constraint $\beta_{f} \leq 75^{\circ}$ was added to the optimization procedure, which is in the range of experimentally obtained mean angles, see Holzapfel [29]. Again, only model 4 and 8 are able to reproduce the experimental curves.

\begin{tabular}{|c|c|c|c|c|c|c|c|}
\hline & $\psi$ in - & $\mu$ in $\mathrm{kPa}$ & $k$ in - & $\mu_{1}$ in $\mathrm{kPa}$ & $k_{1}$ in - & $\beta_{\mathrm{f}}$ in ${ }^{\circ}$ & $f^{\text {obj }}$ \\
\hline 2 & $W_{\mathrm{eH}}+\sum_{a=1}^{2} \psi_{\boldsymbol{\Theta}_{H}}^{\mathrm{ti}}\left(I_{4_{(a)}}^{\mathrm{H}^{1}}\right)$ & 15.90 & 3.50 & $7.4 \mathrm{e}-06$ & 246.90 & 75.00 & 0.127 \\
\hline 4 & $W_{\mathrm{eH}}+\sum_{a=1}^{2} \psi_{\mathbf{\Phi}_{H}}^{\mathrm{ti}}\left(I_{4_{(a)}}^{\mathrm{H}^{2}}\right)$ & 15.90 & $1.0 \mathrm{e}-08$ & 0.05 & 3707.99 & 50.30 & 0.045 \\
\hline 6 & $W_{\mathrm{eH}}+\sum_{a=1}^{2} \psi_{\mathbf{\Phi}_{H}}^{\mathrm{ti}}\left(I_{4_{(a)}}^{\mathrm{H}^{3}}\right)$ & 15.90 & 3.58 & 6.00 & 17045.18 & 63.28 & 0.128 \\
\hline 8 & $W_{\mathrm{eH}}+\sum_{a=1}^{2} \psi_{\boldsymbol{\Phi}_{H}}^{\mathrm{ti}}\left(I_{4_{(a)}}^{\mathrm{H}^{4}}\right)$ & 15.90 & $1.0 \mathrm{e}-08$ & 3973.68 & 56653.51 & 54.74 & 0.071 \\
\hline
\end{tabular}

Table 4: Adjusted parameter sets of the Adventitia. 

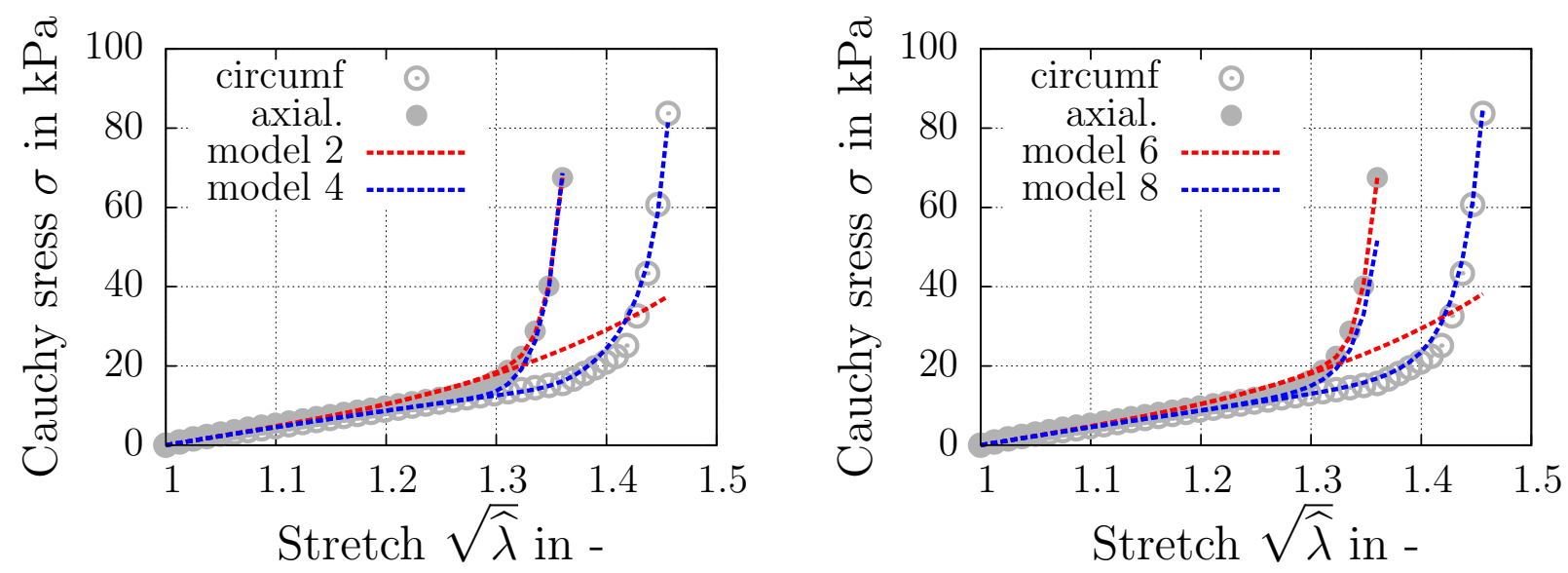

Figure 23: Evolution of the stress ratio induced by different anisotropic measures under biaxial-tension-compression.

\subsection{Artery}

In this section the calibrated transversely isotropic model is applied to three-dimensional simulations of coronary patient-specific arterial walls in order to prove the robustness of the novel material formulation. Since the parameter fitting was based on an diseased abdominal artery, the results may merely be discussed in a qualitative context and conclusion related to clinical interpretation are highly limited. An arterial geometry is reconstructed based on two sequenced two-dimensional virtual histology (VH) intravascular ultrasound (IVUS) images. For a detailed description of the three-dimensional reconstruction the reader is referred to Balzani et al. [7].

The considered artery consists of two layers, see Fig. 24. The outer layer is the Adventitia, the inner layer the Media. This artery was loaded in a finite element simulation with an inner pressure of $16 \mathrm{kPa}$. An augmented Lagrange strategy was applied to enforce quasi-incompressibility with an allowed tolerance of $1 \%$ in change of volume. For additional information the reader is referred to Hestenes [24], Powell [51, Glowinski and Le Tallec [18], Glowinski and Le Tallec [19] and Glowinski and Le Tallec [20]. Tetrahedron finite elements with ten nodes and quadratic shape functions are used.

In a first simulation model 4 , based on $I_{4}^{\mathrm{H}^{2}}$, was used with the estimated parameters from the adjustment, see Table 3 and Table 4 . These results are to be compared with a second simulation, where the strain energy function and parameters according to Schröder and Brinkhues [53] were used. There a Mooney-Rivlin model was used for the isotropic part and the function from Holzapfel et al. [30], given in Eq. (61), for the superimposed transversely isotropic parts. Both parts refer to the strain measure $\boldsymbol{C}$. The parameters were adjusted to the same experimental data. The deformed configurations for different stresses are shown in Fig. 25. While the general stress distributions are comparable the increase in the volume of the lumen is significantly larger for the Hencky model.

The same effect for different strain energy functions, which were adjusted to the same data and then used for numerical simulations of arterial segments was noticed in Brands et al. [10]. 

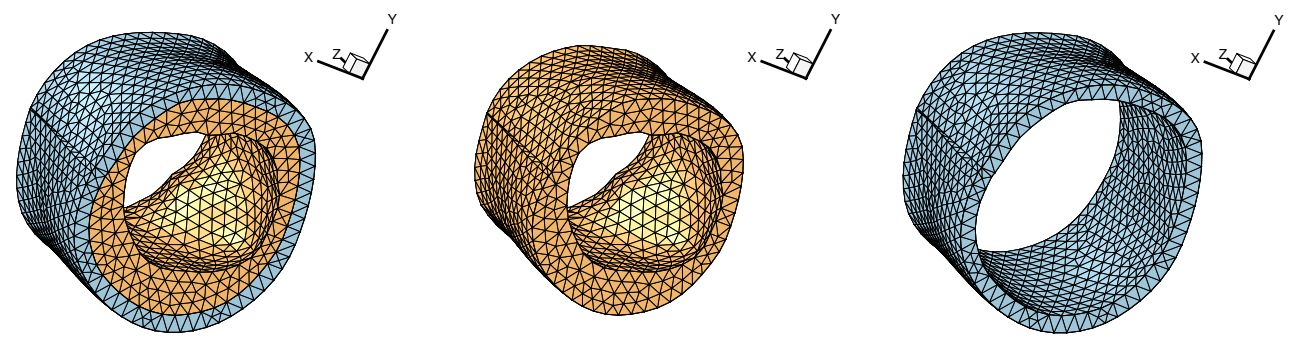

Figure 24: Reconstructed artery consisting of Adventitia (outer layer) and Media (inner layer).

a)

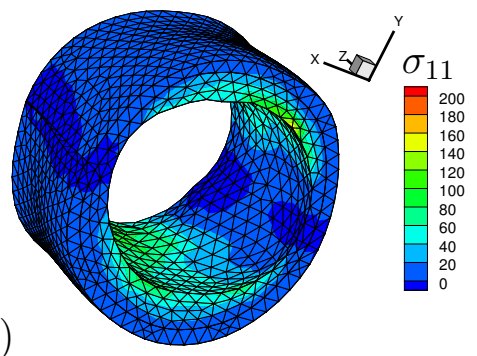

b)

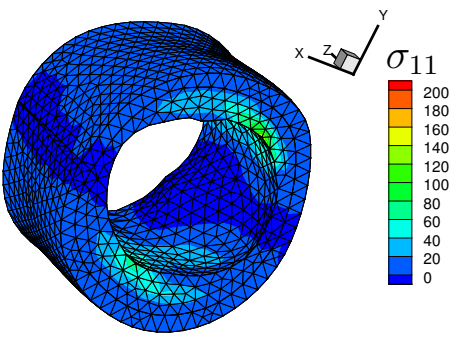

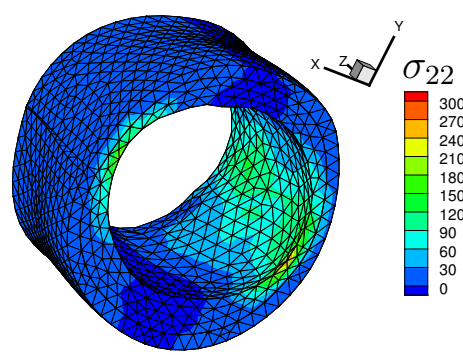

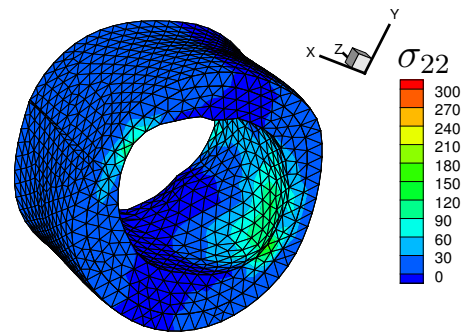

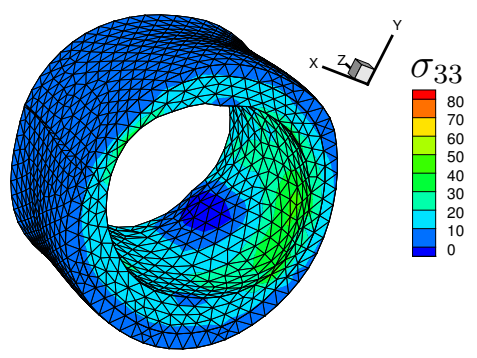

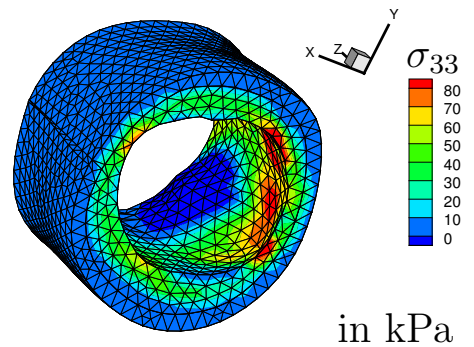

Figure 25: Deformed configuration and stress distributions in an arterial segment, a) simulated with model 4 , based on $I_{4}^{\mathrm{H}^{2}}$ and b) the material model and parameters used in Schröder and Brinkhues [53] based on $I_{4}^{\mathrm{C}^{1}}$ for an internal pressure $p$ of $16 \mathrm{kPa}$.

\section{Open problems}

While the ellipticity domain of the isotropic exponentiated Hencky energy is reasonably well understood (it is an extremely large cone in stretch space) the same is not clear for the anisotropic logarithmic energy terms. The problem which has to be faced is due to the analytical difficulties in treating derivatives of the right Hencky strain tensor; we need to calculate for example $\partial_{\boldsymbol{F}}^{2}\left(\exp \langle\log \boldsymbol{U}, \boldsymbol{M}\rangle^{2}\right) .(\boldsymbol{\xi} \otimes \boldsymbol{\eta}, \boldsymbol{\xi} \otimes \boldsymbol{\eta})$ which is manageable along the lines of the presented algorithmic treatment in this paper; but it remains to identify a suitable ellipticity region. The experience with the isotropic exponentiated Hencky energy suggests, however, that the ellipticity domain largely contains the physical range of arteries, i.e. principal stretches in a suitable $\lambda_{k} \in[1 / 2,2]$. Therefore, our model proposal is elliptic in the physiological range of arteries and this is all that must be required on mathematical grounds. 
ad hoc basis, it remains to study whether the differential-geometric program presented in Neff et al. 48, may be extended to the anisotropic case. Of course, major technical difficulties have to be solved. The benefit, however, cannot be overemphasized: There would result finite strain anisotropic energies having a clear physical meaning. We will pursue this issue in the future.

Acknowledgments The first two authors gratefully acknowledge support by the Deutsche Forschungsgemeinschaft in the Priority Program 1748 under the project "Novel finite elements for anisotropic media at finite strain" (SCHR 570/23-1), section 1 4.3. Further, the first two authors would like to acknowledge support by the Deutsche Forschungsgemeinschaft within the framework of the project "Domain-decomposition-based fluid structure interaction algorithms for highly nonlinear and anisotropic elastic arterial wall models in 3D" (SCHR 570/15-2) under the D-A-CH agreement, section 4.4.5. 


\section{References}

[1] L. Anand. On H. Henckys approximate strain energy function for moderate deformations. Journal of Applied Mechanics, 46:78-82, 1979.

[2] L. Anand. Moderate deformations in extension - torsion of incompressible isotropic elastic materials. Journal of the Mechanics and Physics of Solids, 34:293 304, 1986.

[3] M. Baker and J. L. Ericksen. Inequalities restricting the form of the stressdeformation relations for isotropic elastic solids and Reiner-Rivlin fluids. Journal of the Washington Academy of Sciences, 44:33-35, 1954.

[4] J. M. Ball. Convexity conditions and existence theorems in non-linear elasticity. Archive of Rational Mechanics and Analysis, 63:337-403, 1977.

[5] J. M. Ball. Some open problems in elasticity. In P. Newton, P. Holmes, and A. Weinstein, editors, Geometry, mechanics, and dynamics, pages 3-59. Springer, New-York, 2002 .

[6] D. Balzani, P. Neff, J. Schröder, and G. A. Holzapfel. A polyconvex framework for soft biological tissues. Adjustment to experimental data. International Journal of Solids and Structures, 43:6052-6070, 2006.

[7] D. Balzani, D. Böse, D. Brands, R. Erbel, A. Klawonn, O. Rheinbach, and J. Schröder. Parallel simulation of patient-specific atherosclerotic arteries for the enhancement of intravascular ultrasound diagnostics. Engineering Computations, 29:888-906, 2012.

[8] J. P. Boehler. Lois de comportement anisotrope des milieux continus. Journal de Mécanique, 17:153-190, 1978.

[9] J. P. Boehler. A simple derivation of representations for non-polynomial constitutive equations in some cases of anisotropy. Zeitschrift für angewandte Mathematik und Mechanik, 59:157-167, 1979.

[10] D. Brands, A. Klawonn, O. Rheinbach, and J. Schröder. Modelling and convergence in arterial wall simulations using a parallel FETI solution strategy. Computer Methods in Biomechanics and Biomedical Engineering, 11:569-583, 2008.

[11] O. T. Bruhns, H. Xiao, and A. Meyers. Hencky's elasticity model with the logarithmic strain measure: A study on Poynting effect and stress response in torsion of tubes and rods. Archives of Mechanics, 52:489-509, 2000.

[12] O. T. Bruhns, H. Xiao, and A. Meyers. Constitutive inequalities for an isotropic elastic strain-energy function based on Hencky's logarithmic strain tensor. Proceedings of the Royal Society of London, Series A : Mathematical, Physical and Engineering Science, 457:2207-2226, 2001.

[13] V. Ebbing, J. Schröder, and P. Neff. Approximation of anisotropic elasticity tensors at the reference state with polyconvex energies. Archive of Applied Mechanics, 79: 651-657, 2009. 
[14] A. E. Ehret and M. Itskov. A polyconvex hyperelastic model for fiber-reinforced materials in application to soft tissues. Journal of Material Science, 42:8853-8863, 2007.

[15] P. J. Flory. Thermodynamic relations for high elastic materials. Trans. Faraday Soc., 57:829-838, 1961.

[16] I. D. Ghiba, P. Neff, and R. Martin. An ellipticity domain for the distortional Henckylogarithmic strain energy. Proceedings of the Royal Society of London, Series A : Mathematical, Physical and Engineering Science, 471:20150510, 2015.

[17] I. D. Ghiba, P. Neff, and M. Šilhavỳ. The exponentiated Hencky-logarithmic strain energy. Improvement of planar polyconvexity. International Journal of Non-Linear Mechanics, 71:48-51, 2015.

[18] R. Glowinski and P. Le Tallec. Finite element analysis in nonlinear incompressible elasticity. In J. Oden and G. Carey, editors, Finite elements, Vol V: Special Problems in Solid Mechanics. Prentice-Hall, Englewood Cliffs, NH, 1984.

[19] R. Glowinski and P. Le Tallec. Augmented Lagrangian methods for the solution of variational problems. Springer, Berlin, 1988.

[20] R. Glowinski and P. Le Tallec. Augmented Lagrangian and operator-splitting methods in nonlinear mechanics, volume 9 of SIAM Studies in Applied Mathematics. Society for Industrial and Applied Mathematics (SIAM), Philadelphia, PA, 1989.

[21] S. Hartmann and P. Neff. Polyconvexity of generalized polynomial-type hyperelastic strain energy functions for near-incompressibility. International Journal of Solids and Structures, 40:2767-2791, 2003.

[22] H. Hencky. Über die Form des Elastizitätsgesetzes bei ideal elastischen Stoffen. Zeitschrift für technische Physik, 9:215-220, 1928.

[23] H. Hencky. Welche Umstände bedingen die Verfestigung bei der bildsamen Verformung von festen isotropen Körpern? Zeitschrift für Physik, 55:145-155, 1929.

[24] M. Hestenes. Multiplier and gradient methods. Journal of Optimization Theory and Applications, 4:303-320, 1969.

[25] R. Hill. On constitutive inequalities for simple materials. Journal of the Mechanics and Physics of Solids, 16:229-242, 1968.

[26] R. Hill. Constitutive inequalities for isotropic elastic solids under finite strain. Proceedings of the Royal Society of London, Series A : Mathematical, Physical and Engineering Science, 314:457-472, 1970.

[27] R. Hill. Aspects of invariance in solid mechanics. Advances in Applied Mechanics, 18:1-75, 1978.

[28] A. Hoger. The stress conjugate to logarithmic strain. International Journal of Solids and Structures, 23:1645-1656, 1987. 
[29] G. A. Holzapfel. Determination of material models for arterial walls from uniaxial extension tests and histological structure. Journal of Theoretical Biology, 238:290302, 2006.

[30] G. A. Holzapfel, T. C. Gasser, and R. W. Ogden. A new constitutive framework for arterial wall mechanics and a comparative study of material models. Journal of Elasticity, 61:1-48, 2000.

[31] S. K. Hyun and H. Nakajima. Anisotropic compressive properties of porous copper produced by unidirectional solidification. Material Science and Engineering, A340: 258-264, 2003.

[32] M. Itskov, A. E. Ehret, and D. Mavrilas. A polyconvex anisotropic strain-energy function for soft collagenous tissues. Biomechanics and Modelling in Mechanobiology, $5: 17-26,2006$.

[33] C. S. Jog. Derivatives of the stretch, rotation and exponential tensors in ndimensional vector spaces. Journal of Elasticity, 82:175-192, 2006.

[34] C. S. Jog and K. D. Patil. Conditions for the onset of elastic and material instabilities in hyperelastic materials. Archive of Applied Mechanics, 83(5):661-684, 2013.

[35] M. Latorre and F. J. Montáns. Anisotropic finite strain viscoelasticity based on the Sidoroff multiplicative decomposition and logarithmic strains. Computational Mechanics, 56:503-531, 2015.

[36] J. Löblein, J. Schröder, and F. Gruttmann. Application of generalized measures to an orthotropic finite elasto-plasticity model. Computational Materials Science, 28: 696-703, 2003.

[37] R. J. Martin and P. Neff. Minimal geodesics on GL(n) for left-invariant, right-O(n)invariant Riemannian metrics. Journal of Geometric Mechanics, 8(3):323-357, 2016.

[38] L. A. Mihai and P. Neff. Hyperelastic bodies under homogeneous Cauchy stress induced by three-dimensional non-homogeneous deformations. to appear in Mathematics and Mechanics of Solids, 2017.

[39] L. A. Mihai and P. Neff. Hyperelastic bodies under homogeneous Cauchy stress induced by non-homogeneous finite deformations. International Journal of NonLinear Mechanics, 89:93-100, 2017.

[40] G. Montella, S. Govindjee, and P. Neff. The exponentiated Hencky strain energy in modelling tire derived material for moderately large deformations. Journal of Engineering Materials and Technology, 138:031008, 2016.

[41] P. Neff. Mathematische Analyse multiplikativer Viskoplastizität. Ph.D. Thesis, Technische Universität Darmstadt. Shaker Verlag, Aachen, 2000.

[42] P. Neff and I. D. Ghiba. The exponentiated Hencky-logarithmic strain energy. Part III: Coupling with idealized isotropic finite strain plasticity. Continuum Mechanics and Thermodynamics, 28:477-487, 2016. 
[43] P. Neff and L. A. Mihai. Injectivity of the Cauchy-stress tensor along rank-one connected lines under strict rank-one convexity condition. to appear in Journal of Elasticity, 2016.

[44] P. Neff, B. Eidel, F. Osterbrink, and R. J. Martin. The Hencky strain energy $\|\log U\|^{2}$ measures the geodesic distance of the deformation gradient to $\mathrm{SO}(3)$ in the canonical left-invariant Riemannian metric on GL(3). Proceedings of Applied Mathematics and Mechanics, 13:369-370, 2013.

[45] P. Neff, B. Eidel, and R. J. Martin. The axiomatic deduction of the quadratic Hencky strain energy by Heinrich Hencky. arXiv:1402.4027, 2014.

[46] P. Neff, I. Ghiba, and J. Lankeit. The exponentiated Hencky-logarithmic strain energy. Part I: Constitutive issues and rank-one convexity. Journal of Elasticity, 121: 143-234, 2015.

[47] P. Neff, J. Lankeit, I. D. Ghiba, R. J. Martin, and D. J. Steigmann. The exponentiated Hencky-logarithmic strain energy. Part II: Coercivity, planar polyconvexity and existence of minimizers. Zeitschrift für angewandte Mathematik und Physik, 66: 1671-1693, 2015.

[48] P. Neff, B. Eidel, and R. J. Martin. Geometry of logarithmic strain measures in solid mechanics. Archive of Rational Mechanics and Analysis, 222:507-572, 2016.

[49] R. W. Ogden. Large deformation isotropic elasticity - On the correlation of theory and experiment for incompressible rubberlike solids,. Proceedings of the Royal Society London A, 326:565584, 1972.

[50] R. W. Ogden. Non-Linear elastic deformations. Courier Corporation, 1997.

[51] M. Powell. A method for nonlinear constraints in minimization problems. In R. Fletcher, editor, Optimization, pages 283-298. Academic Press, New York, 1969.

[52] H. Richter. Das isotrope Elastizitätsgesetz. Zeitschrift für angewandte Mathematik und Mechanik, 28:205-209, 1948.

[53] J. Schröder and S. Brinkhues. A novel scheme for the computation of residual stresses in arterial walls. Archive of Applied Mechanics, 84:881-898, 2014.

[54] J. Schröder and D. Gross. Invariant formulation of the electromechanical enthalpy function of transversely isotropic piezoelectric materials. Archive of Applied Mechanics, 73:533-552, 2004.

[55] J. Schröder and P. Neff. Invariant formulation of hyperelastic transverse isotropy based on polyconvex free energy functions. International Journal of Solids and Structures, 40:401-445, 2003.

[56] J. Schröder, F. Gruttmann, and J. Löblein. A simple orthotropic finite elastoplasticity model based on generalized stress-strain measures. Computational Mechanics, 30:4864, 2002. 
[57] J. Schröder, P. Neff, and D. Balzani. A variational approach for materially stable anisotropic hyperelasticity. International Journal of Solids and Structures, 42(15): 4352-4371, 2005.

[58] J. Schröder, P. Neff, and V. Ebbing. Anisotropic polyconvex energies on the basis of crystallographic motivated structural tensors. Journal of the Mechanics and Physics of Solids, 56:3486-3506, 2008.

[59] J. C. Simo. Numerical Analysis and Simulation of Plasticity, Vol. 6 of Handbook of Numerical Analysis. Elsevier Science, 1998.

[60] A. J. M. Spencer. Kinematic constraints, constitutive equations and failure rules for anisotropic materials. In J. P. Boehler, editor, Applications of Tensor Functions in Solid Mechanics, volume 292 of CISM Courses and Lectures, pages 187-197. Springer, 1987.

[61] C. Vallée. Lois de comportement élastique isotropes en grandes déformations. International Journal of Engineering Science, 16:451-457, 1978. 


\section{A Appendix}

\section{A.1 Notes on the Hencky tensor}

The Hencky strain tensor is defined through

$$
\log \boldsymbol{U}=\frac{1}{2} \log \boldsymbol{C}
$$

and

$$
\operatorname{tr}(\log \boldsymbol{U})=\log (\operatorname{det} \boldsymbol{U})
$$

The symmetric right Cauchy-Green tensor $\boldsymbol{C}$ in spectral decomposition is given by

$$
\boldsymbol{C}=\sum_{k=1}^{3} \widehat{\lambda}_{k} \boldsymbol{N}^{k} \otimes \boldsymbol{N}^{k}=\sum_{k=1}^{3} \widehat{\lambda}_{k} \boldsymbol{P}^{k}, \quad \text { with } \quad \boldsymbol{P}^{k}=\boldsymbol{N}^{k} \otimes \boldsymbol{N}^{k}
$$

and for the Hencky strain we obtain

$$
\log \boldsymbol{U}=\sum_{k=1}^{3} \frac{1}{2} \log \left(\widehat{\lambda}_{k}\right) \boldsymbol{N}^{k} \otimes \boldsymbol{N}^{k}=\sum_{k=1}^{3} \frac{1}{2} \log \left(\widehat{\lambda}_{k}\right) \boldsymbol{P}_{k}, \quad \text { with } \quad \boldsymbol{P}_{k}=\boldsymbol{N}^{k} \otimes \boldsymbol{N}^{k}
$$

The first derivative of $\log \boldsymbol{U}$ with respect to $\boldsymbol{C}$ can be computed as

$$
\begin{aligned}
\frac{\partial \log \boldsymbol{U}}{\partial \boldsymbol{C}} & =\sum_{k=1}^{3} \boldsymbol{P}_{k} \otimes \frac{\partial \frac{1}{2} \log \widehat{\lambda}_{k}}{\partial \boldsymbol{C}}+\frac{1}{2} \log \widehat{\lambda}_{k} \frac{\partial \boldsymbol{P}_{k}}{\partial \boldsymbol{C}} \\
& =\sum_{k=1}^{3} \boldsymbol{P}_{k} \otimes \frac{\partial \frac{1}{2} \log \widehat{\lambda}_{k}}{\partial \widehat{\lambda}_{k}} \frac{\partial \widehat{\lambda}_{k}}{\partial \boldsymbol{C}}+\frac{1}{2} \log \widehat{\lambda}_{k} \frac{\partial \boldsymbol{P}_{k}}{\partial \boldsymbol{C}} \\
& =\sum_{k=1}^{3} \frac{1}{2} \widehat{\lambda}_{k}^{-1} \boldsymbol{P}_{k} \otimes \frac{\partial \widehat{\lambda}_{k}}{\partial \boldsymbol{C}}+\frac{1}{2} \log \widehat{\lambda}_{k} \frac{\partial \boldsymbol{P}_{k}}{\partial \boldsymbol{C}}
\end{aligned}
$$

Considering that

$$
\frac{\partial \widehat{\lambda}_{k}}{\partial \boldsymbol{C}}=\boldsymbol{P}_{k} \quad \text { and } \quad \frac{\partial \boldsymbol{P}_{k}}{\partial \boldsymbol{C}}=\sum_{j=1, j \neq k}^{3} \frac{\boldsymbol{P}_{k} \otimes \boldsymbol{P}_{j}^{T}+\boldsymbol{P}_{j} \otimes \boldsymbol{P}_{k}^{T}}{\widehat{\lambda}_{k}-\widehat{\lambda}_{j}},
$$

see for instance Jog [33], we find that

$$
\frac{\partial \log \boldsymbol{U}}{\partial \boldsymbol{C}}=\sum_{k=1}^{3} \frac{1}{2} \widehat{\lambda}_{k}^{-1} \boldsymbol{P}_{k} \otimes \boldsymbol{P}_{k}+\sum_{k=1}^{3} \sum_{\substack{j=1 \\ k \neq j}}^{3} \frac{\frac{1}{2}\left(\log \widehat{\lambda}_{k}\right)-\frac{1}{2}\left(\log \widehat{\lambda}_{j}\right)}{\widehat{\lambda}_{k}-\widehat{\lambda}_{j}}\left(\boldsymbol{P}_{k} \otimes \boldsymbol{P}_{j}+\boldsymbol{P}_{j} \otimes \boldsymbol{P}_{k}\right)
$$


The second derivative for the linearization is given by

$$
\begin{aligned}
\frac{\partial^{2} \log \boldsymbol{U}}{\partial \boldsymbol{C} \partial \boldsymbol{C}}= & \sum_{k=1}^{3} \boldsymbol{P}_{k} \otimes \boldsymbol{P}_{k} \otimes \frac{\partial \frac{1}{2} \widehat{\lambda}_{k}^{-1}}{\partial \boldsymbol{C}}+\frac{1}{2} \widehat{\lambda}_{k}^{-1}\left[\left(\frac{\partial \boldsymbol{P}_{k}}{\partial \boldsymbol{C}} \otimes \boldsymbol{P}_{k}\right)^{3546}+\boldsymbol{P}_{k} \otimes \frac{\partial \boldsymbol{P}_{k}}{\partial \boldsymbol{C}}\right] \\
& +\frac{1}{2}\left(\log \widehat{\lambda}_{k}\right) \frac{\partial^{2} \boldsymbol{P}_{k}}{\partial \boldsymbol{C} \partial \boldsymbol{C}}+\frac{\partial \boldsymbol{P}_{k}}{\partial \boldsymbol{C}} \otimes \frac{\partial \frac{1}{2} \widehat{\lambda}_{k}^{-1}}{\partial \boldsymbol{C}} \\
= & \sum_{k=1}^{3}-\frac{1}{2} \widehat{\lambda}_{k}^{-2} \boldsymbol{P}_{k} \otimes \boldsymbol{P}_{k} \otimes \boldsymbol{P}_{k}+\frac{1}{2} \widehat{\lambda}_{k}^{-1}\left[\left(\frac{\partial \boldsymbol{P}_{k}}{\partial \boldsymbol{C}} \otimes \boldsymbol{P}_{k}\right)^{\frac{3546}{T}}+\boldsymbol{P}_{k} \otimes \frac{\partial \boldsymbol{P}_{k}}{\partial \boldsymbol{C}}\right] \\
& +\frac{1}{2}\left(\log \widehat{\lambda}_{k}\right) \frac{\partial^{2} \boldsymbol{P}_{k}}{\partial \boldsymbol{C} \partial \boldsymbol{C}}+\frac{1}{2} \widehat{\lambda}_{k}^{-1} \frac{\partial \boldsymbol{P}_{k}}{\partial \boldsymbol{C}} \otimes \boldsymbol{P}_{k},
\end{aligned}
$$

where

$$
\begin{aligned}
\frac{\partial^{2} \boldsymbol{P}_{k}}{\partial \boldsymbol{C} \partial \boldsymbol{C}}= & \sum_{\substack{j=1 \\
k \neq j}}^{3} \frac{1}{\widehat{\lambda}_{k}-\widehat{\lambda}_{j}}\left[\left(\boldsymbol{P}_{j}^{T} \otimes\left(\frac{\partial \boldsymbol{P}_{k}}{\partial \boldsymbol{C}}\right)^{\frac{12}{T}}\right)^{23}+\left(\boldsymbol{P}_{k} \otimes \frac{\partial \boldsymbol{P}_{j}}{\partial \boldsymbol{C}}\right)^{23}\right. \\
& \left.+\left(\boldsymbol{P}_{k}^{T} \otimes\left(\frac{\partial \boldsymbol{P}_{j}}{\partial \boldsymbol{C}}\right)^{\frac{12}{T}}\right)^{\frac{23}{T}}+\left(\boldsymbol{P}_{j} \otimes \frac{\partial \boldsymbol{P}_{k}}{\partial \boldsymbol{C}}\right)^{\frac{23}{T}}\right] \\
& +\frac{1}{\left(\widehat{\lambda}_{k}-\widehat{\lambda}_{j}\right)^{2}}\left(\boldsymbol{P}_{k} \otimes \boldsymbol{P}_{j}+\boldsymbol{P}_{j} \otimes \boldsymbol{P}_{k}\right) \otimes\left(\boldsymbol{P}_{j}-\boldsymbol{P}_{k}\right) .
\end{aligned}
$$

The exponent and the logarithm of an arbitrary symmetric tensor may also be be expressed with help of a Taylor expansion of the form

$$
\begin{aligned}
& \exp (\bullet)=\mathbf{1}+\sum_{m=1}^{\infty} \frac{1}{m !}(\bullet)^{m} \quad \text { and } \\
& \log (\bullet)=\sum_{k=1}^{\infty} \frac{-1^{k-1}}{k}[(\bullet)-\mathbf{1}]^{k},
\end{aligned}
$$

where the latter is convergent in a neighborhood of $\mathbf{1}$.

\section{A.2. Conjugate stress tensors}

The following considerations are adapted from Ogden [50].

The constitutive equation for the stresses are derived form the (isothermal) entropy inequality

$$
\dot{\psi}-\langle\boldsymbol{P}, \dot{\boldsymbol{F}}\rangle \geq 0,
$$

From the latter we deduce the constitutive relation $\boldsymbol{P}=\partial_{\boldsymbol{F}} \psi$. Let the generalized Lagrangean strain measures

$$
\boldsymbol{E}^{(m)}= \begin{cases}\frac{1}{2}\left(\boldsymbol{U}^{m}-\mathbf{1}\right) & m \neq 0 \\ \frac{1}{2} \log \boldsymbol{U} & m=0\end{cases}
$$


and Eulerian strain measures

$$
\boldsymbol{K}^{(m)}= \begin{cases}\frac{1}{m}\left(\boldsymbol{V}^{m}-\mathbf{1}\right) & m \neq 0 \\ \log \boldsymbol{V} & m=0\end{cases}
$$

be given, we aim to find the corresponding constitutive equations. The so called stress power may be written as

$$
\langle\boldsymbol{P}, \dot{\boldsymbol{F}}\rangle=\langle\boldsymbol{\tau}, \boldsymbol{D}\rangle=\langle\boldsymbol{S}, \dot{\boldsymbol{E}}\rangle=\left\langle\partial_{\boldsymbol{E}^{(m)}} \psi, \dot{\boldsymbol{E}}^{(m)}\right\rangle=\left\langle\partial_{\boldsymbol{K}^{(m)}} \psi, \dot{\boldsymbol{K}}^{(m)}\right\rangle,
$$

where $\boldsymbol{D}=\frac{1}{2}\left(\boldsymbol{L}+\boldsymbol{L}^{T}\right)$ and $\boldsymbol{L}=\operatorname{grad} \dot{\boldsymbol{x}}$. Considering that $\dot{\boldsymbol{E}}=\frac{1}{2}\left(\dot{\boldsymbol{F}}^{T} \boldsymbol{F}+\boldsymbol{F}^{T} \dot{\boldsymbol{F}}\right)$, we obtain the relations

$$
\boldsymbol{P}=\boldsymbol{F} \boldsymbol{S}=\boldsymbol{\tau} \boldsymbol{F}^{-T} .
$$

The pairs in Eq. (88) are said to be work conjugate. By making use of the fact that $\boldsymbol{R}^{T} \dot{\boldsymbol{R}}=-\dot{\boldsymbol{R}}^{T} \boldsymbol{R}$, we may rewrite $\dot{\boldsymbol{E}}=\frac{1}{2}(\boldsymbol{U} \dot{\boldsymbol{U}}+\dot{\boldsymbol{U}} \boldsymbol{U})$ and we are able to reformulate

$$
\left\langle\boldsymbol{S}, \frac{1}{2}(\boldsymbol{U} \dot{\boldsymbol{U}}+\dot{\boldsymbol{U} U})\right\rangle=\langle\underbrace{\frac{1}{2}(\boldsymbol{S U}+\boldsymbol{U} \boldsymbol{S})}_{\boldsymbol{T}_{\text {Biot }}}, \dot{\boldsymbol{U}}\rangle,
$$

such that we directly obtain the Biot stress $\boldsymbol{T}_{\text {Biot }}=\partial_{\boldsymbol{U}} \psi^{\#}(\boldsymbol{U})$, work conjugate to $\boldsymbol{U}$ from the entropy inequality. With $\boldsymbol{U}=\boldsymbol{R}^{T} \boldsymbol{V} \boldsymbol{R}$ we are able to relate $\boldsymbol{E}^{(m)}$ and $\boldsymbol{K}^{(m)}$ and the corresponding time derivatives as follows:

$$
\boldsymbol{E}^{(m)}=\boldsymbol{R}^{T} \boldsymbol{K}^{(m)} \boldsymbol{R} \text { and } \quad \dot{\boldsymbol{E}}^{(m)}=\boldsymbol{R}^{T} \dot{\boldsymbol{K}}^{(m)} \boldsymbol{R}+\boldsymbol{E}^{(m)} \boldsymbol{R}^{T} \dot{\boldsymbol{R}}-\boldsymbol{R}^{T} \dot{\boldsymbol{R}} \boldsymbol{E}^{(m)} .
$$

Regarding the generalized stress-power it follows

$$
\left\langle\partial_{\boldsymbol{E}^{(m)}} \psi, \dot{\boldsymbol{E}}^{(m)}\right\rangle=\left\langle\boldsymbol{R}\left(\partial_{\boldsymbol{E}^{(m)}} \psi\right) \boldsymbol{R}^{T}, \dot{\boldsymbol{K}}^{(m)}\right\rangle+\left\langle\left[\left(\partial_{\boldsymbol{E}^{(m)}} \psi\right) \boldsymbol{E}^{(m)}-\boldsymbol{E}^{(m)}\left(\partial_{\boldsymbol{E}^{(m)}} \psi\right)\right], \boldsymbol{R}^{T} \dot{\boldsymbol{R}}\right\rangle
$$

Only if $\boldsymbol{E}^{(m)} \partial_{\boldsymbol{E}^{(m)}} \psi=\partial_{\boldsymbol{E}^{(m)}} \psi \boldsymbol{E}^{(m)}$, i.e. $\partial_{\boldsymbol{E}^{(m)}} \psi$ is coaxial with $\boldsymbol{E}^{(m)}$, it immediately follows that the constitutive law results in

$$
\frac{\partial \psi}{\partial \boldsymbol{K}^{(m)}}=\boldsymbol{R}\left(\partial_{\boldsymbol{E}^{(m)}} \psi\right) \boldsymbol{R}^{T}
$$

and the stress power is expressed through $\left\langle\dot{\boldsymbol{K}}^{(m)}, \boldsymbol{R}\left(\partial_{\boldsymbol{E}^{(m)}} \psi\right) \boldsymbol{R}^{T}\right\rangle$, which is identical to Eq. 88.).

The case that $\partial_{\boldsymbol{E}^{(m)}} \psi$ is coaxial with $\boldsymbol{E}^{(m)}$ implies that also $\partial_{\boldsymbol{E}^{(m)}} \psi$ and $\boldsymbol{U}$ are coaxial. Under this assumption one may show that

$$
\partial_{\boldsymbol{E}^{(m)}} \psi=\boldsymbol{T}_{\text {Biot }} \boldsymbol{U}^{-(m-1)}=\boldsymbol{U}^{-(m-1)} \boldsymbol{T}_{\text {Biot }},
$$

and it follows that

$$
\partial_{\boldsymbol{E}^{(m)}} \psi=\boldsymbol{T}_{\text {Biot }} \boldsymbol{U}^{-m+1} \quad \text { and } \quad \partial_{\boldsymbol{E}^{(0)}} \psi=\partial_{\log \boldsymbol{U}} \psi=\boldsymbol{T}_{\text {Biot }} \boldsymbol{U}=\boldsymbol{R}^{T} \boldsymbol{\tau} \boldsymbol{R},
$$

for isotropic materials. Inserting the latter result in Eq. 93 we obtain the relation

$$
\boldsymbol{\tau}=\frac{\partial \psi(\log \boldsymbol{V})}{\partial \log \boldsymbol{V}}
$$

Regarding the conjugate stress to $\log \boldsymbol{U}$ the reader is also referred to Hill [26; 27] and Hoger [28]. 